\title{
Sato Grassmannian and Degenerate Sigma Function
}

\author{
Julia Bernatska, ${ }^{*}$ Victor Enolski ${ }^{\dagger}$ and Atsushi Nakayashiki ${ }^{\ddagger}$
}

Dedicated to Mikio Sato on his 90th birthday

\begin{abstract}
The degeneration of the hyperelliptic sigma function is studied. We use the Sato Grassmannian for this purpose. A simple decomposition of a rational function gives a decomposition of Plücker coordinates of a frame of the Sato Grassmannian. It then gives a decomposition of the tau function corresponding to the degeneration of a hyperelliptic curve of genus $g$ in terms of the tau functions corresponding to a hyperelliptic curve of genus $g-1$. Since the tau functions are described by sigma functions, we get the corresponding formula for the degenerate hyperelliptic sigma function.
\end{abstract}

\section{Introduction}

We study an algebraic curve of genus $g>1$ and the associated sigma-function of $g$ variables which represents a natural generalization of the Weierstrass elliptic sigma function to curves with higher genus. Solutions of KP type equations are built of this function by known rules [17. In particular they can be given as logarithmic derivatives of the sigmafunction. Degenerate solutions appear when the curves has multiple points, in particular, double points. The simplest degenerate genus $g$ solution, which is studied in this paper, can be understood as a soliton on the background of multi-phase wave packet expressed in terms of the hyperbolic function and the sigma function of $g-1$ variables. Such solutions are in great demands of applied sciences and explicit formulae in closed form are requested.

Here we deal with the case of hyperelliptic curves. But the method first manifested in [23. on the example of trigonal curve has a wider area of applicability. Thus we consider hyperelliptic case as a good laboratory for further generalizations. The main features of the method are the following.

First we show that the Sato theory, commonly considered as a deep theoretical description of integrability, can be implemented for the derivation of explicit formulae in a very concrete problem - the construction of degenerate solutions in an explicit and closed form.

\footnotetext{
*National University of Kyiv-Mohyla Academy, H. Skovorody St. 2, 04655 Kyiv, Ukraine, jbernatska@gmail.com

${ }^{\dagger}$ National University of Kyiv-Mohyla Academy, H. Skovorody St. 2, 04655 Kyiv, Ukraine, venolski@googlemail.com

${ }^{\ddagger}$ Department of Mathematics, Tsuda University, Kodaira, Tokyo 187-8577, Japan, atsushi@tsuda.ac.jp
} 
In the case considered the hyperellptic curve with a double point leads to the decomposition of the Grassmannian frame and the decomposition of the tau function of genus $g$ to the tau functions of genus $g-1$.

Next, we incorporate the viewpoint of [5] and further developments, in particular, [26, 17, 11, 20, 25, 22, 6, 18, 2], that the sigma function provides an algebraic counterpart of the Riemann theta function and an effective language to deal with the algebro-geometric integrability of soliton equations. After [21, 12, 10] the tau function is expressed in terms of the sigma function and the interrelation of sigma and tau is a part of the construction.

Also our derivation is based on the notion of Schottky-Klein bidifferential whose fundamental role in the construction of Abelian function associated with Riemann surfaces is elucidated in [14]. If we represent the tau function by Riemann's theta function, the additional exponential term is written using the expansion coefficients of the normalized bidifferential which is expressed through the Riemann theta function [29]. On the other hand if we represent the tau function by the sigma function the exponential term is written by expansion coefficients of the non-normalized algebraic bidifferential [21, 10]. For hyperelliptic curves such representation of the bidifferential is known from H.Baker's monograph [3] and nowadays it is obtained for wider classes of curves [8, 20, 28, 13. To derive the formula for the degenerate sigma function we need to calculate the difference of bidifferentials corresponding to the degeneration of a genus $g$ curve and the curve of genus $g-1$. We demonstrate that, with the help of the explicit algebraic expression of the bidifferential, it can neatly be expressed in terms of the coefficients of the defining equation of the curve. In this way we get the formula which expresses the degenerate sigma function of genus $g$ in terms of the sigma functions of genus $g-1$. The case of genus two was previously studied in [4, where the system of linear differential equations satisfied by the sigma function was used to derive the formula. Our result generalizes it to the case of genus $g$ by another method (see section 6 for details).

Here it should be emphasized that the method proposed here permits to circumvent calculations of limits of the Riemann matrix and the whole procedure reduces to routine expansions of algebraic quantities such as the non-normalized bidifferential represented in algebraic form. Thus we can expect that it is effective for the more complicated curves than hyperelliptic curves.

Recently degenerations of curves to genus $g=0$ and associated trigonometric solutions to the KP hierarchies were studied in [16, 1] ( see also references therein). It is shown that the Sato Grassmannian approach is effective in this case [23, 24]. We believe that our consideration will permit us to extend interesting results and observation found in these publications to the case of genus $g>1$ degeneration of a curve.

The organization of the paper is as follows. In section 2 we recall the Sato Grassmannian and the tau function of the KP-hierarchy. The points of the Sato Grassmannian corresponding to a hyperelliptic curves are studied in section 3. By decomposing the frame corresponding to a degenerate hyperellptic curve, the decomoposition of the degenerate tau function is derived. In section 4 the formula of the tau function in terms of sigma function is derived. Detailed properties of the hyperelliptic sigma functions are used to do it. The formula for the degenerate sigma function of genus $g$ in terms of the sigma function of genus $g-1$ is derived using the formula in the previous sections in section 5 . Finally, in section 6 , we show that the formula of [4] for the degeneration of the sigma function of genus two can be derived by the method in this paper. 


\section{Sato Grassmannian and tau function}

We denote by $\mathbb{Z}$ the set of integers and by $\mathbb{N}$ the set of positive integers.

Let

$$
V=\mathbb{C}((z))=\left\{\sum_{-\infty<<i<+\infty} a_{i} z^{i} \mid a_{i} \in \mathbb{C}\right\}
$$

be the vector space of Laurent series in $z$ and

$$
V_{\emptyset}=\mathbb{C}\left[z^{-1}\right], \quad V_{0}=z \mathbb{C}[[z]] .
$$

Then

$$
V=V_{\emptyset} \oplus V_{0}
$$

Define the projection map from $V$ to $V_{\emptyset}$ by $\pi$ :

$$
\pi: V \rightarrow V / V_{0} \simeq V_{\emptyset}
$$

The Sato Grassmannian UGM is defined as the set of subspaces $U$ of $V$ such that $\operatorname{Ker}\left(\left.\pi\right|_{U}\right)$ and $\operatorname{Coker}\left(\pi_{U}\right)$ are finite dimensional and their dimensions are equal.

Example 2.1. $U=V_{\emptyset} \in$ UGM. In this case $\operatorname{dim} \operatorname{Ker}(\pi \mid U)=\operatorname{dim} \operatorname{Coker}(\pi \mid U)=0$.

Example 2.2. $U=\mathbb{C} z+\sum_{i=0}^{\infty} \mathbb{C} z \wp^{(i)}(z) \in$ UGM, where $\wp(z)$ is the Weierstrass elliptic function and $\wp^{(i)}(z)=\mathrm{d}^{i} \wp(z) / \mathrm{d} z^{i}$. In this case $\operatorname{dim} \operatorname{Ker}(\pi \mid U)=\operatorname{dim} \operatorname{Coker}(\pi \mid U)=1$.

We set

$$
e_{i}=z^{i+1}
$$

A basis $\xi=\left(\ldots, v_{3}, v_{2}, v_{1}\right)$ of $U \in \mathrm{UGM}$ is called a frame of $U$. Write

$$
v_{j}=\sum_{-\infty<<i<+\infty} \xi_{i, j} e_{i}
$$

and identify $v_{j}$ with the column vector $\left(\xi_{i, j}\right)_{i \in \mathbb{Z}}$,

$$
v_{j}=\left[\begin{array}{c}
\vdots \\
\xi_{-1, j} \\
\xi_{0, j} \\
\xi_{1, j} \\
\vdots
\end{array}\right]
$$

Then $\xi$ can be expressed as a $\mathbb{Z} \times \mathbb{N}$ matrix $\left(\xi_{i, j}\right)_{i \in \mathbb{Z}, j \in \mathbb{N}}$.

For any point $U$ of UGM it is always possible to take a frame in the following form: there exists $l \geq 1$ such that, for $j \geq l$,

$$
v_{j}=e_{-j}+\sum_{i>-j} \xi_{i, j} e_{i}
$$


In the matrix form it means that the frame has the following form

$$
\xi=\left[\begin{array}{cccccc}
\ddots & O & \vdots & \vdots & & \vdots \\
\cdots & 1 & 0 & * & \cdots & * \\
\cdots & * & 1 & * & \cdots & * \\
\vdots & \vdots & \vdots & \vdots & & \vdots
\end{array}\right]
$$

where the right most 1 in $(2.2)$ is the $(-l, l)$ component. In the following we assume that a frame is always of this form.

A sequence of integers $M=\left(m_{1}, m_{2}, \ldots\right)$ such that

$$
\begin{aligned}
& m_{1}>m_{2}>\cdots, \\
& m_{j}=-j \quad \forall j>>0,
\end{aligned}
$$

is called a Maya diagram. The set of Maya diagrams and that of partitions bijectively correspond to each other by

$$
\begin{aligned}
M=\left(m_{1}, m_{2}, \ldots\right) & \longrightarrow \lambda(M)=\left(m_{1}+1, m_{2}+2, \ldots\right), \\
\lambda=\left(\lambda_{1}, \lambda_{2}, \ldots\right) & \longrightarrow M(\lambda)=\left(\lambda_{1}-1, \lambda_{2}-2, \ldots\right) .
\end{aligned}
$$

For a partition $\lambda$ the Plücker coordinate $\xi_{\lambda}$ of a frame $\xi$ is defined by

$$
\begin{aligned}
& \xi_{\lambda}=\operatorname{det}\left(\xi_{m_{i}, j}\right)_{i, j \in \mathbb{N}}, \\
& M(\lambda)=\left(m_{1}, m_{2}, \ldots\right) .
\end{aligned}
$$

The infinite determinant is well defined for a frame of the form (2.2).

The Schur function $s_{(i)}(t), t=\left(t_{1}, t_{2}, \ldots\right)^{t}$, corresponding to the partition $(i), i \geq 0$, is defined by

$$
\exp \left(\sum_{i=1}^{\infty} t_{i} k^{i}\right)=\sum_{i=0}^{\infty} s_{(i)}(t) k^{i} .
$$

For an arbitrary partition $\lambda=\left(\lambda_{1}, \lambda_{2}, \ldots, \lambda_{l}\right)$ the Schur function $s_{\lambda}(t)$ is defined by

$$
s_{\lambda}(t)=\operatorname{det}\left(s_{\left(\lambda_{i}-i+j\right)}(t)\right)_{1 \leq i, j \leq l} .
$$

Assign the weight $i$ for $t_{i}$. Then $s_{\lambda}(t)$ is homogeneous of the weight $|\lambda|=\sum \lambda_{i}$.

The tau function of a frame $\xi$ of a point $U$ of UGM is defined by

$$
\tau(t ; \xi)=\sum_{\lambda} \xi_{\lambda} s_{\lambda}(t)
$$

where the sum is over all partitions. If $\xi^{\prime}$ is another frame of $U$ then $\tau\left(t ; \xi^{\prime}\right)$ is a constant multiple of $\tau(t ; \xi)$.

The tau function gives a solution of the KP-hierarchy. The KP-hierarchy in the bilinear form is the equation for $\tau(t), t=\left(t_{1}, t_{2}, \ldots\right)^{t}$ given by

$$
\oint \mathrm{e}^{-2 \sum_{j=1}^{\infty} s_{j} z^{j}} \tau\left(t-s-\left[z^{-1}\right]\right) \tau\left(t+s+\left[z^{-1}\right]\right) \frac{\mathrm{d} z}{2 \pi i}=0,
$$


where $s=\left(s_{1}, s_{2}, s_{3}, \ldots\right)^{t},[z]=\left(z, z^{2} / 2, z^{3} / 3, \ldots\right)$ and the integral signifies to take the coefficient of $z^{-1}$ in the formal series expansion of the integrand in $z$.

For a solution $\tau(t)$ of the KP-hierarchy $u(t)=2 \partial_{t_{1}}^{2} \log \tau(t)$ satisfies the KP equation

$$
3 u_{t_{2} t_{2}}+\left(-4 u_{t_{3}}+6 u u_{t_{1}}+u_{t_{1} t_{1} t_{1}}\right)_{t_{1}}=0
$$

Sato's fundamental theorem tells that any formal power series solutions of the KPhierarchy can be constructed from UGM.

Theorem 2.3. 27. For any $U \in$ UGM and a frame $\xi$ of $U \tau(t ; \xi)$ is a solution of the KP-hierarchy. Conversely for any formal power series solution $\tau(t)$ of the KP-hierarchy there exists a unique point $U$ of UGM and a frame $\xi$ of $U$ such that $\tau(t)=\tau(t ; \xi)$.

\section{Tau functions of hyperelliptic curves}

\subsection{The case of zero point}

Consider the hyperelliptic curve $C$ of genus $g$ given by

$$
y^{2}=\prod_{j=1}^{2 g+1}\left(x-\alpha_{j}\right)
$$

where $\left\{\alpha_{i}\right\}$ are mutually distinct complex numbers. The space of meromorphic functions on $C$ which are regular on $C \backslash\{\infty\}$ is denoted by $H^{0}(C, \mathcal{O}(* \infty))$.

A basis of this vector space is given by

$$
x^{i}, \quad i \geq 0, \quad x^{i} y, \quad i \geq 0 .
$$

We take the local coordinate $z$ around $\infty$ such that

$$
x=z^{-2}, \quad y=z^{-(2 g+1)} F(z), \quad F(z)=\left(\prod_{j=1}^{2 g+1}\left(1-\alpha_{j} z^{2}\right)\right)^{1 / 2} .
$$

Then the basis (3.2) is written as

$$
z^{-2 i}, \quad i \geq 0, \quad z^{-2 g-1-2 i} F(z), \quad i \geq 0 .
$$

We consider $F(z)$ as a power series in $z$ by the Taylor expansion at $z=0$. Multiplying these functions by $z^{g}$ we get a frame of UGM (see $\S 4$ of [24])

$$
z^{g-2 i}, \quad i \geq 0, \quad z^{-g-1-2 i} F(z), \quad i \geq 0 .
$$

If a Laurent series $v(z)$ is of the form

$$
v(z)=\sum_{n \geq N} a_{n} z^{n}, \quad a_{N} \neq 0,
$$

we define the order of $v(z)$ to be $-N$. Arrange elements of (3.4) by their orders and denote them by $\widetilde{f}_{1}, \widetilde{f}_{2}, \ldots$ Explicitly

$$
\left(\widetilde{f}_{1}, \ldots, \widetilde{f}_{g}\right)=\left(z^{g}, z^{g-2}, z^{g-4}, \ldots, z^{-g+2}\right),
$$




$$
\begin{aligned}
& \widetilde{f}_{g+2 i+1}=z^{-g-2 i}, \quad i \geq 0 \\
& \widetilde{f}_{g+2 i+2}=z^{-g-1-2 i} F(z), \quad i \geq 0 .
\end{aligned}
$$

Define the frame $\tilde{X}$ of a point of UGM by

$$
\widetilde{X}=\left[\ldots, \widetilde{f}_{3}, \widetilde{f}_{2}, \widetilde{f}_{1}\right] .
$$

Notice that $\widetilde{X}$ satisfies (2.1).

\subsection{Degeneration}

Let $\alpha$ be a complex number which is different from $\alpha_{j}, 1 \leq j \leq 2 g-1$. We consider the limit of $C$ when $\alpha_{2 g}$ and $\alpha_{2 g+1}$ go to $\alpha$. The curve $C$ tends to

$$
y^{2}=(x-\alpha)^{2} \prod_{j=1}^{2 g-1}\left(x-\alpha_{j}\right)
$$

The limit of the function $F(z)$ becomes

$$
F(z)=\left(1-\alpha z^{2}\right) F_{0}(z), \quad F_{0}(z)=\left(\prod_{j=1}^{2 g-1}\left(1-\alpha_{j} z^{2}\right)\right)^{1 / 2}
$$

Then the limit of $\widetilde{f}_{j}$ exists and it is obtained by replacing $F(z)$ by $\left(1-\alpha z^{2}\right) F_{0}(z)$ in $\widetilde{f}_{j}$. Denote the limit of $\widetilde{f}_{j}$ by $\widetilde{f}_{j}^{0}$. Define $\widetilde{X}_{0}$ as the limit of the frame $\widetilde{X}$,

$$
\widetilde{X}_{0}=\left[\ldots, \widetilde{f}_{3}^{0}, \widetilde{f}_{2}^{0}, \widetilde{f}_{1}^{0}\right]
$$

It still is a frame of a point of a UGM, since the highest order terms of $f_{j}$ and $\widetilde{f}_{j}^{0}$ are the same. Let $\widetilde{U}_{0}$ be the corresponding point of UGM.

We multiply $\widetilde{X}_{0}$ by $\left(1-\alpha z^{2}\right)^{-1}$. Then the following set of functions is a basis of $\left(1-\alpha z^{2}\right)^{-1} \widetilde{U}_{0}$

$$
\frac{z^{g-2 i}}{1-\alpha z^{2}}, \quad i \geq 0, \quad z^{-g-1-2 i} F_{0}(z), \quad i \geq 0 .
$$

Lemma 3.1. We have the following equation

$$
\left\langle\frac{z^{g-2 i}}{1-\alpha z^{2}}, \quad i \geq 0\right\rangle=\left\langle\frac{z^{g}}{1-\alpha z^{2}}, z^{g-2-2 i}, \quad i \geq 0\right\rangle,
$$

where, for a set of functions $S,<S>$ denotes the vector space generated by $S$.

Proof. Notice that $z^{g} /\left(1-\alpha z^{2}\right)$ belongs to both the LHS and the RHS of the equation (3.8). Then using the relation

$$
\frac{z^{g-2 i}-\alpha z^{g-2(i-1)}}{1-\alpha z^{2}}=z^{g-2 i}
$$


it is easy to show that the RHS is included in the LHS by inducion on $i$. The converse inclusion relation can be proved by using

$$
\frac{z^{g-2(i+1)}}{1-\alpha z^{2}}=z^{g-2(i+1)}+\frac{\alpha z^{g-2 i}}{1-\alpha z^{2}}
$$

By Lemma 3.1 and (3.7) the set of functions

$$
\frac{z^{g}}{1-\alpha z^{2}}, \quad z^{g-2-2 i}(i \geq 0), \quad z^{-g-1-2 i} F_{0}(z)(i \geq 0) .
$$

becomes a basis of $\left(1-\alpha z^{2}\right)^{-1} \widetilde{U}_{0}$. Arrange the functions in (3.10) by their orders and denote them by $f_{1}, f_{2}, \ldots$,

$$
\begin{aligned}
\left(f_{1}, f_{2}, f_{3}, \ldots, f_{g}\right) & =\left(\frac{z^{g}}{1-\alpha z^{2}}, z^{g-2}, z^{g-4}, \ldots, z^{-g+2}\right) \\
f_{g+2 i+1} & =z^{-g-2 i}, \quad i \geq 0, \\
f_{g+2 i+2} & =z^{-g-2 i-1} F_{0}(z), \quad i \geq 0 .
\end{aligned}
$$

Define the frame $X$ of UGM by

$$
X=\left(X_{i, j}\right)_{i \in \mathbb{Z}, j \in \mathbb{N}}=\left[\ldots, f_{3}, f_{2}, f_{1}\right] .
$$

In the change from (3.7) to (3.10) only the relation (3.9) is used. It does not change the Plücker coordinates. Therefore the tau functions of $X$ and $\left(1-\alpha z^{2}\right)^{-1} \widetilde{X}_{0}$ are the same,

$$
\tau(t ; X)=\tau\left(t ;\left(1-\alpha z^{2}\right)^{-1} \widetilde{X}_{0}\right) .
$$

\subsection{The case of one point}

Consider the hyperelliptic curve $C^{\prime}$ of genus $g-1$ given by

$$
y^{2}=\prod_{j=1}^{2 g-1}\left(x-\alpha_{j}\right)
$$

which is extracted from the singular curve (3.6).

Let $z$ be the local coordinate of $C^{\prime}$ around $\infty$ such that

$$
x=z^{-2}, \quad y=z^{-(2 g+1)} F_{0}(z)
$$

and $\alpha$ be as in the previous section.

Fix a point $\left(\alpha, y_{0}\right)$ of $C^{\prime}$ and set

$$
p_{ \pm}=\left(\alpha, \pm y_{0}\right)
$$

Since $\alpha$ is different from $\alpha_{j}, 1 \leq j \leq 2 g-1, y_{0} \neq 0$ and $p_{+} \neq p_{-}$.

For a point $P$ of $C^{\prime}$ denote by $H^{0}\left(C^{\prime}, \mathcal{O}(P+* \infty)\right)$ the space of meromorphic functions on $C^{\prime}$ which are regular on $C^{\prime} \backslash\{P, \infty\}$ and have a pole at $P$ of order at most one. Define the vector spaces $W_{ \pm}$by

$$
W_{ \pm}=H^{0}\left(C^{\prime}, \mathcal{O}\left(p_{ \pm}+* \infty\right)\right)
$$


The following set functions is a basis of $W_{ \pm}$,

$$
x^{i}, \quad i \geq 0, \quad x^{i} y, \quad i \geq 0, \quad \frac{y \pm y_{0}}{x-\alpha} .
$$

Writing these functions in $z$ and multiply them by $z^{g-2}$ we get

$$
z^{g-2-2 i}, \quad i \geq 0, \quad z^{-g-1-2 i} F_{0}(z), \quad i \geq 0, \quad z^{-g+1} \frac{F_{0}(z) \pm y_{0} z^{2 g-1}}{1-\alpha z^{2}} .
$$

It becomes a frame of a point of UGM (see $\S 4$ of [24]). Arrange the functions in (3.14) by their orders and name them as $h_{1}^{ \pm}, h_{2}^{ \pm}, \ldots$,

$$
\begin{aligned}
\left(h_{1}^{ \pm}, h_{2}^{ \pm}, \ldots, h_{g}^{ \pm}\right) & =\left(z^{g-2}, z^{g-4}, \ldots, z^{-g+2}, z^{-g+1} \frac{F_{0}(z) \pm y_{0} z^{2 g-1}}{1-\alpha z^{2}}\right) \\
h_{g+2 i+1}^{ \pm} & =z^{-g-2 i}, \quad i \geq 0, \\
h_{g+2 i+2}^{ \pm} & =z^{-g-1-2 i} F_{0}(z), \quad i \geq 0 .
\end{aligned}
$$

We define the frames $Y^{ \pm}$of points of UGM by

$$
Y^{ \pm}=\left[\ldots, h_{3}^{ \pm}, h_{2}^{ \pm}, h_{1}^{ \pm}\right]
$$

\subsection{Relation of tau functions}

Since $h_{i}^{+}$and $h_{i}^{-}$coincide except $i=g$, we set, for $i \neq g$,

$$
h_{i}=h_{i}^{+}=h_{i}^{-} \text {. }
$$

Then $h_{i}$ is related to $f_{i}$ by

$$
h_{i}= \begin{cases}f_{i+1} & 1 \leq i \leq g-1 \\ f_{i} & i \geq g+1\end{cases}
$$

The following important decomposition holds,

$$
f_{1}=\left(2 y_{0}\right)^{-1}\left(h_{g}^{+}-h_{g}^{-}\right) .
$$

Substituting (3.17) into the frame $X$, for any partition $\lambda$, the Plücker coordinate $X_{\lambda}$ can be written in terms of the Plücker coordinates $Y_{\lambda}^{ \pm}$as

$$
X_{\lambda}=(-1)^{g-1}\left(2 y_{0}\right)^{-1}\left(Y_{\lambda}^{+}-Y_{\lambda}^{-}\right) .
$$

Thus we have

\section{Theorem 3.2.}

$$
\tau(t ; X)=(-1)^{g-1}\left(2 y_{0}\right)^{-1}\left(\tau\left(t ; Y^{+}\right)-\tau\left(t ; Y^{-}\right)\right) .
$$




\section{Tau function in terms of sigma function}

\subsection{Hyperelliptic sigma function}

Write the right hand side of (3.1) as

$$
\prod_{j=1}^{2 g+1}\left(x-\alpha_{j}\right)=\sum_{i=0}^{2 g+1} \mu_{4 g+2-2 i} x^{i}, \quad \mu_{0}=1 .
$$

We assign the weight $k$ to $\mu_{k}$, and weight 2 to $x$. Then this polynomial is homogenous of the weight $4 g+2$.

Let $\left\{\mathfrak{a}_{i}, \mathfrak{b}_{i} \mid 1 \leq i \leq g\right\}$ be a canonical basis of the homology group of $C,\left\{\mathrm{~d} u_{2 i-1}^{(g)} \mid 1 \leq\right.$ $i \leq g\}$ a basis of holomorphic one forms given by

$$
\mathrm{d} u_{2 i-1}^{(g)}=\frac{x^{g-i} \mathrm{~d} x}{-2 y} .
$$

and $\widehat{\Omega}^{(g)}\left(p_{1}, p_{2}\right)$ the bidifferential on $C \times C$ defined by

$$
\begin{aligned}
\widehat{\Omega}^{(g)}\left(p_{1}, p_{2}\right) & =\frac{2 y_{1} y_{2}+\sum_{i=0}^{g} x_{1}^{i} x_{2}^{i}\left(2 \mu_{4 g+2-4 i}+\mu_{4 g-4 i}\left(x_{1}+x_{2}\right)\right)}{4\left(x_{1}-x_{2}\right)^{2} y_{1} y_{2}} \mathrm{~d} x_{1} \mathrm{~d} x_{2} \\
& =\mathrm{d}_{p_{2}}\left(\frac{y_{1}+y_{2}}{2 y_{1}\left(x_{1}-x_{2}\right)}\right)+\sum_{i=1}^{g} \mathrm{~d} u_{2 i-1}^{(g)}\left(p_{1}\right) \mathrm{d} r_{2 i-1}^{(g)}\left(p_{2}\right),
\end{aligned}
$$

where $p_{i}=\left(x_{i}, y_{i}\right) \in C$ and

$$
\mathrm{d} r_{2 i-1}^{(g)}=\frac{\mathrm{d} x}{-2 y} \sum_{k=1}^{2 i-1} k \mu_{4 i-2 k-2} x^{k+g-i} .
$$

Define the $g \times g$ period matrices by

$$
\begin{array}{rlrl}
2 \omega^{(g)} & =\left(\int_{\mathfrak{a}_{j}} \mathrm{~d} u_{2 i-1}^{(g)}\right), & 2 \omega^{\prime(g)}=\left(\int_{\mathfrak{b}_{j}} \mathrm{~d} u_{2 i-1}^{(g)}\right), \\
-2 \eta^{(g)} & =\left(\int_{\mathfrak{a}_{j}} \mathrm{~d} r_{2 i-1}^{(g)}\right), & & -2 \eta^{\prime(g)}=\left(\int_{\mathfrak{b}_{j}} \mathrm{~d} r_{2 i-1}^{(g)}\right), \\
T^{(g)} & =\left(\omega^{(g)}\right)^{-1} \omega^{\prime(g)} . &
\end{array}
$$

Set

$$
\begin{aligned}
& m_{k}^{(g)}=\left[\frac{g+1-k}{2}\right], \quad 0 \leq k \leq g, \\
& \left(w_{1}, \ldots, w_{g}\right)=(1,3,5, \ldots, 2 g-1), \quad\left(w_{1}^{*}, \ldots, w_{g}^{*}\right)=(0,2,4, \ldots, 2 g-2) .
\end{aligned}
$$

For $0 \leq k \leq g-1$ define

$$
A_{k}^{(g)}=\left(a_{k, 1}^{(g)}, \ldots, a_{k, m_{k}}^{(g)}\right)=(2 g-2 k-1,2 g-2 k-5,2 g-2 k-9, \ldots),
$$




$$
\mathrm{s}_{k}^{(g)}=\operatorname{sgn}\left(\begin{array}{cccccc}
w_{1}^{*} & \ldots & w_{m_{k}}^{*} & w_{g-k-m_{k}^{(g)}} & \ldots & w_{1} \\
g-k-1 & & \ldots & \ldots & 1 & 0
\end{array}\right) .
$$

We set $A_{g}^{(g)}=\emptyset, c_{g}^{(g)}=1$. The following relation is valid,

$$
A_{k}^{(g)}=A_{0}^{(g-k)} .
$$

Example 4.1. $A_{2}^{(3)}=A_{1}^{(2)}=A_{0}^{(1)}=(1)$,

$A_{2}^{(4)}=A_{1}^{(3)}=A_{0}^{(2)}=(3)$,

$A_{2}^{(5)}=A_{1}^{(4)}=A_{0}^{(3)}=(5,1)$,

$A_{2}^{(6)}=A_{1}^{(5)}=A_{0}^{(4)}=(7,3)$.

Remark 4.2. The number $A_{k}^{(g)}$ is introduced in [26] by different notation for hyperelliptic curves. Its generalizations are given by [25] for $(n, s)$ curves and by [22] for arbitrary Riemann surfaces.

In general, for $A=\left(a_{1}, \ldots, a_{r}\right) \in\{1,3, \ldots, 2 g-1\}^{r}$ and a function $G(u), u=\left(u_{1}, u_{3}\right.$, $\left.\ldots, u_{2 g-1}\right)^{t}$, set

$$
\begin{aligned}
& G_{A}(u)=\partial_{u_{a_{1}}} \cdots \partial_{u_{a_{r}}} G(u), \quad \partial_{u_{a}}=\frac{\partial}{\partial u_{a}}, \\
& |A|=a_{1}+\cdots+a_{r} .
\end{aligned}
$$

For $A=\emptyset$ set $G_{A}(u)=G(u)$.

For $\varepsilon, \varepsilon^{\prime} \in \mathbb{R}^{g}$ let

$$
\theta\left[\begin{array}{c}
\varepsilon \\
\varepsilon^{\prime}
\end{array}\right]\left(v \mid T^{(g)}\right)=\sum_{n \in \mathbb{Z}^{g}} \exp \left(\pi i\left(n+\varepsilon^{\prime}\right)^{t} T^{(g)}\left(n+\varepsilon^{\prime}\right)+2 \pi i\left(n+\varepsilon^{\prime}\right)^{t}(v+\varepsilon)\right)
$$

be the Riemann's theta function with characteristics. In the context of the problem considered characteristics $\varepsilon, \varepsilon^{\prime} \in \mathbb{R}^{g}$ are half integers, $\varepsilon_{i}, \varepsilon_{j}^{\prime}=0$ or $1 / 2$. Choose $\infty$ as a base point and denote by $K$ the Riemann's constant for this choice, then characteristics of vector $-K$, denoted as $[-K]$, are known to be half-integers. The following is valid

Theorem 4.3. [25] $\theta[-K]_{A_{0}^{(g)}}\left(0 \mid T^{(g)}\right) \neq 0$.

Set $u=\left(u_{1}, u_{3}, \ldots, u_{2 g-1}\right)^{t}$. Then the fundamental sigma function for $\left(C, \infty,\left\{\mathrm{d} u_{2 i-1}^{(g)}\right\}, \widehat{\Omega}^{(g)}\right)$ is defined by

$$
\sigma^{(g)}(u)=\exp \left(\frac{1}{2} u^{t} \eta^{(g)}\left(\omega^{(g)}\right)^{-1} u\right) \frac{\theta[-K]\left(\left(2 \omega^{(g)}\right)^{-1} u \mid T^{(g)}\right)}{\mathrm{s}_{0}^{(g)} \theta[-K]_{A_{0}^{(g)}}\left(0 \mid T^{(g)}\right)} .
$$

Assign the weight $-i$ to $u_{i}$.

The sigma function does not depend on the choice of a canonical homology basis and has the expansion of the form

$$
\sigma(u)=s_{\nu^{(g)}}(u)+(\text { terms with weights }>|\nu|),
$$

where $\nu^{(g)}=(g, g-1, \ldots, 1)$. 


\subsection{Tau function corresponding to the frame $\tilde{X}$}

Define $b_{i j}^{(g)}, \widehat{q}_{i j}^{(g)}$ and $c_{i}^{(g)}$ by the expansions at $\infty$,

$$
\begin{aligned}
\mathrm{d} u_{2 i-1}^{(g)} & =\left(\sum_{j=1}^{\infty} b_{i j}^{(g)} z^{j-1}\right) \mathrm{d} z \\
\widehat{\Omega}^{(g)}\left(p_{1}, p_{2}\right) & =\left(\frac{1}{\left(z_{1}-z_{2}\right)^{2}}+\sum_{i, j=1}^{\infty} \widehat{q}_{i j}^{(g)} z_{1}^{i-1} z_{2}^{j-1}\right) \mathrm{d} z_{1} \mathrm{~d} z_{2}, \\
\log \left(z^{-g+1} \sqrt{\frac{\mathrm{d} u_{2 g-1}^{(g)}}{\mathrm{d} z}}\right) & =\sum_{i=1}^{\infty} c_{i}^{(g)} \frac{z^{i}}{i} .
\end{aligned}
$$

Using

$$
\mathrm{d} u_{2 i-1}^{(g)}=\frac{z^{2 i-2} \mathrm{~d} z}{F(z)}
$$

$c_{i}^{(g)}$ can be expressed explicitly by $\left\{\alpha_{j}\right\}$ as follows. We have

$$
\begin{aligned}
\log \left(z^{-g+1} \sqrt{\frac{\mathrm{d} u_{2 g-1}^{(g)}}{\mathrm{d} z}}\right) & =-\frac{1}{2} \log F(z) \\
& =-\frac{1}{4} \sum_{j=1}^{2 g+1} \log \left(1-\alpha_{j} z^{2}\right) \\
& =\sum_{i=1}^{\infty}\left(\frac{1}{2} \sum_{j=1}^{2 g+1} \alpha_{j}^{i}\right) \frac{z^{2 i}}{2 i}
\end{aligned}
$$

Thus

$$
c_{i}^{(g)}= \begin{cases}0 & \text { if } i \text { is odd } \\ \frac{1}{2} \sum_{j=1}^{2 g+1} \alpha_{j}^{i / 2} & \text { if } i \text { is even. }\end{cases}
$$

Define

$$
B^{(g)}=\left(b_{i j}^{(g)}\right)_{1 \leq i \leq g, j \geq 1}, \quad \widehat{q}^{(g)}(t)=\sum_{i, j=1}^{\infty} \widehat{q}_{i j}^{(g)} t_{i} t_{j} .
$$

Then

Theorem 4.4. 21] The tau function corresponding to the frame $\widetilde{X}$ is given by

$$
\tau(t ; \widetilde{X})=\exp \left(-\sum_{i=1}^{\infty} c_{2 i}^{(g)} t_{2 i}+\frac{1}{2} \widehat{q}^{(g)}(t)\right) \sigma^{(g)}\left(B^{(g)} t\right) .
$$




\subsection{Tau function corresponding to the frames $Y^{ \pm}$}

In this section $p_{ \pm}=\left(\alpha, \pm y_{0}\right)$ denote the points on $C$ except in Theorem 4.9, where $C^{\prime}$ is considered.

Set

$$
\mathrm{d} u^{(g)}=\left(\mathrm{d} u_{1}^{(g)}, \mathrm{d} u_{3}^{(g)}, \ldots, \mathrm{d} u_{2 g-1}^{(g)}\right)^{t} .
$$

For points $p_{1}, \ldots, p_{k}$ on $C$ and any $A=\left(a_{1}, \ldots, a_{r}\right)$, we set

$$
\left.{ }^{\left(\sigma^{(g)}\right.}\right)_{A}\left(p_{1}+\cdots+p_{k}\right)=\left(\sigma^{(g)}\right)_{A}\left(\int_{\infty}^{p_{1}} \mathrm{~d} u^{(g)}+\cdots+\int_{\infty}^{p_{k}} \mathrm{~d} u^{(g)}\right)
$$

for the sake of simplicity.

Proposition 4.5. 26] Let $1 \leq k \leq g$.

(i) As a function of $p_{1}, \ldots, p_{k} \in C,\left(\sigma^{(g)}\right)_{A_{k}^{(g)}}\left(p_{1}+\cdots+p_{k}\right)$ is not identically zero.

(ii) The following expansion in $z_{k}=z\left(p_{k}\right)$ holds,

$$
\begin{aligned}
\left.{ }^{\left(\sigma^{(g)}\right)}\right)_{A_{k}^{(g)}}\left(p_{1}+\cdots+p_{k}\right) & \\
& =\mathrm{s}_{k, k-1}^{(g)}\left(\sigma^{(g)}\right){ }_{A_{k-1}^{(g)}}\left(p_{1}+\cdots+p_{k-1}\right) z_{k}^{g+1-k}+O\left(z_{k}^{g+2-k}\right),
\end{aligned}
$$

where $\mathrm{s}_{k, k-1}^{(g)}=\mathrm{s}_{k}^{(g)} \mathrm{s}_{k-1}^{(g)}$.

Since $p_{+}$is the image of $p_{-}$by the hyperelliptic involution $(x, y) \rightarrow(x,-y)$,

$$
\int_{\infty}^{p_{-}} \mathrm{d} u^{(g)}=-\int_{\infty}^{p_{+}} \mathrm{d} u^{(g)}
$$

Therefore, we have, by the case $k=2$ of Proposition (4.5) (ii),

$$
\sigma_{A_{2}^{(g)}}^{(g)}\left(p-p_{ \pm}\right)=\mathrm{s}_{2,1}^{(g)} \sigma_{A_{1}^{(g)}}^{(g)}\left(-p_{ \pm}\right) z^{g-1}+O\left(z^{g}\right)
$$

where $z=z(p)$.

Lemma 4.6. 26] $\sigma_{A_{1}^{(g)}}^{(g)}\left(-p_{ \pm}\right) \neq 0$.

Proof. Set

$$
f(p)=\sigma_{A_{1}^{(g)}}^{(g)}(p)
$$

By Corollary 4 of [25]

$$
f(p)=\mathrm{s}_{1}^{(g)} z^{g}+O\left(z^{g+1}\right),
$$

and consequently $f(p)$ is not identically zero as a function of $p$. By Theorem 8 of [25] $f(p)$ has the same quasi-periodicity (automorphic) factor as that of $\sigma^{(g)}(u)$ substituted by $u=\int_{\infty}^{p} \mathrm{~d} u^{(g)}$. Then by a similar argument to the proof for that the Riemann's theta 
function has $g$ zeros on a Riemann surface, it can be proved that $f(p)$ has $g$ zeros. But $f(p)$ has already a zero of order $g$ at $\infty$ by (4.8). Thus $f(p)$ does not vanish at $p \neq \infty$.

By (4.7) and Lemma 4.6 the following definition of the numbers $d_{j}^{(g, \pm)}, j \geq 1$, makes sense,

$$
\log \left(z^{g-1} \frac{\mathrm{s}_{2,1}^{(g)} \sigma_{A_{1}^{(g)}}^{(g)}\left(-p_{ \pm}\right)}{\sigma_{A_{2}^{(g)}}^{(g)}\left(p-p_{ \pm}\right)}\right)-\frac{1}{2} \widehat{q}^{(g)}([z])=\sum_{j=1}^{\infty} d_{j}^{(g, \pm)} \frac{z^{j}}{j}
$$

where, for $g=1$, we set $A_{2}^{(1)}=\emptyset, \mathrm{s}_{2,1}^{(1)}=1$.

Lemma 4.7. Suppose that $q \in C \backslash\{\infty\}$. Then

$$
\sigma^{(g)}(u+q)=c s_{\nu^{(g-1)}}(u)+\left(\text { terms with the weight }>\frac{1}{2} g(g-1)\right),
$$

for some constant $c \neq 0$, where $\nu^{(g-1)}=(g-1, g-2, \ldots, 1,0)$.

Proof. For $g=1$ there is nothing to prove. Suppose that $g \geq 2$. Take

$$
e=q+(g-2) \infty-\Delta,
$$

in (5) of [22]. We denote by $L$ the holomorphic line bundle associated with

$$
e-(g-1) \infty=q-\infty,
$$

which is denoted by $L_{e+\delta}$ in [22]. Write $q=\left(x_{0}, y_{0}\right)$. Then

$$
x^{i}, \quad x^{i} y, \quad \frac{y+y_{0}}{x-x_{0}}, \quad i \geq 0
$$

is a basis of

$$
H^{0}\left(C, \mathcal{O}(q+* \infty) \simeq H^{0}(C, L(* \infty)) .\right.
$$

Notice that the filtration of the latter space is defined by

$$
H^{0}(C, L(n \infty)) \simeq H^{0}(C, \mathcal{O}(q+(n-1) \infty)) .
$$

Then

$$
\frac{y+y_{0}}{x-x_{0}} \in H^{0}(C, L(2 g \infty)),
$$

since it has a pole of order $2 g-1$ at $\infty$.

Therefore non-gaps of $L$ at $\infty$ are

$$
(1,3,5, \ldots,, 2 g-1,2 g, 2 g+1,2 g+2, \ldots),
$$

and consequently the gap sequence is

$$
(0,2,4, \ldots, 2 g-2) \text {. }
$$

Let $\nu$ be the partition defined by (see Definition 2 of [22])

$$
\nu=(2 g-2,2 g-4, \ldots, 2,0)-(g-1, g-2, \ldots, 1,0)=(g-1, g-2, \ldots, 1,0) .
$$

Then the lemma follows from Theorem 13 of [22]. 
Lemma 4.8. The constant $c$ of Lemma 4.7 is given by

$$
c=\mathrm{s}_{0}^{(g-1)} \sigma_{A_{0}^{(g-1)}}^{(g)}(q) .
$$

For $g=1$ it should be understood that $\mathrm{s}_{0}^{(0)}=1, A_{0}^{(0)}=\emptyset$.

Proof. By Theorem 1 of [25]

$$
\partial_{A_{0}^{(g-1)}} s_{\nu^{(g-1)}}(0)=\mathrm{s}_{0}^{(g-1)} .
$$

Differentiating (4.10) by $\partial_{A_{0}^{(g-1)}}$, using Theorem 8 of [25] and putting $u=0$, we get

$$
\partial_{A_{0}^{(g-1)}} \sigma^{(g)}(q)=c \mathrm{~s}_{0}^{(g-1)}
$$

which proves the lemma.

Then

Theorem 4.9. The tau function corresponding to the frames $Y^{ \pm}$is given by

$$
\tau\left(t ; Y^{ \pm}\right)=\exp \left(\sum_{j=1}^{\infty} d_{j}^{(g-1, \pm)} t_{j}+\frac{1}{2} \widehat{q}^{(g-1)}(t)\right) \frac{\sigma^{(g-1)}\left(B^{(g-1)} t-p_{ \pm}\right)}{\mathrm{s}_{0}^{(g-2)} \sigma_{A_{0}^{(g-2)}}^{(g-1)}\left(-p_{ \pm}\right)} .
$$

Proof. Let $\tau(t)$ be the right hand side of (4.11). We shall calculate

$$
\Psi(t ; z):=\frac{\tau(t+[z])}{\tau(t)} \exp \left(-\sum_{i=1}^{\infty} t_{i} z^{-i}\right)
$$

Let $\mathrm{d} \widetilde{r}_{i}$ be the differential of the second kind with a pole only at $\infty$ such that

$$
\begin{aligned}
& \mathrm{d} \widetilde{r}_{i}=\mathrm{d}\left(\frac{1}{z^{i}}+O(1)\right) \quad \text { near } \infty \\
& \int_{\mathfrak{a}_{j}} \mathrm{~d} \widetilde{r}_{i}=0 \text { for any } j .
\end{aligned}
$$

Set

$$
\mathrm{d} \widehat{r}_{i}=\mathrm{d} \widetilde{r}_{i}+\sum_{k, l=1}^{g} b_{k, i}\left(\eta^{(g)}\left(\omega^{(g)}\right)^{-1}\right)_{k, l} \mathrm{~d} u_{2 l-1}^{(g)}
$$

By Lemma 8 of 21$]$

$$
\mathrm{d} \widehat{r}_{i}=\mathrm{d}\left(\frac{1}{z^{i}}-\sum_{j=1}^{\infty} \widehat{q}_{i, j} \frac{z^{j}}{j}\right)
$$

Then we have

$$
\Psi(t ; z)=z^{g-2} \frac{\mathrm{s}_{2,1}^{(g-1)} \sigma_{A_{1}^{(g-1)}}^{(g-1)}\left(-p_{ \pm}\right)}{\sigma_{A_{2}^{(g-1)}}^{(g-1)}\left(p-p_{ \pm}\right)} \frac{\sigma^{(g-1)}\left(B^{(g-1)} t+p-p_{ \pm}\right)}{\sigma^{(g-1)}\left(B^{(g-1)} t-p_{ \pm}\right)} \exp \left(-\sum_{i=1}^{\infty} t_{i} \int^{p} \mathrm{~d} \widehat{r}_{i}\right)
$$


where $\int^{p} \mathrm{~d} \widehat{r}_{i}$ is the indefinite integral without a constant term in the expansion of $z$. By (4.7) and Lemma 4.6 $\sigma_{A_{2}^{(g-1)}}^{(g-1)}\left(p-p_{ \pm}\right)$does not vanish identically as a function of $p \in C^{\prime}$. By Theorem 8 of [25] it has the same automorphic factor as that of $\sigma\left(u-p_{ \pm}\right)$substituted by $u=\int_{\infty}^{p} \mathrm{~d} u^{(g-1)}$. Therefore $z^{-(g-2)} \Psi(t ; z)$ is a one valued meromorphic function on $C \backslash\{\infty\}$ with an essential singularity at $\infty$, where it has the form,

$$
\Psi(t ; z)=(1+O(z)) \exp \left(-\sum_{i=1}^{\infty} t_{i} z^{-i}\right)
$$

by (4.7). Let us determine the pole of $\Psi(t ; z)$ on $C^{\prime} \backslash\{\infty\}$.

Lemma 4.10. The zero divisor of $\sigma_{A_{2}^{(g-1)}}^{(g-1)}\left(p-p_{ \pm}\right)$is $p_{ \pm}+(g-2) \infty$.

Proof. Let $f(p)$ the function in question. By Theorem 5, Lemma 1 of [25] $f\left(p_{ \pm}\right)=0$. By (4.7) $f(p)$ has a zero of order $g-2$ at $p=\infty$. By a similar argument to the proof of Lemma 4.6 $f(p)$ has $g-1$ zeros on $C^{\prime}$. Thus the zero divisor of $f(p)$ is $p_{ \pm}+(g-2) \infty$.

By the lemma and Krichever's theory [17] $z^{-(g-2)} \Psi(t ; z)$ is the Baker-Akhiezer function corresponding to the divisor $p_{ \pm}+(g-2) \infty$, which is not a general divisor if $g \geq 4$, and $\Psi(t ; z)$ is the adjoint wave function of the KP-hierarchy [9]. So $\tau(t)$ is a solution of the KP-hierarchy. Since $z^{-(g-2)} \Psi(t ; z)$ has a pole at $p_{ \pm}$of order at most one, the expansion coefficients of $\Psi(t ; z)$ in $t_{1}, t_{2}, \ldots$ are contained in the space spanned by $Y^{ \pm}$. Thus the points of UGM corresponding to $\tau(t)$ and $\tau\left(t ; Y^{ \pm}\right)$coincide. Then $\tau(t)$ is a constant multiple of $\tau\left(t ; Y^{ \pm}\right)$by Theorem 2.3 .

By Lemma 4.7, Lemma 4.8 the Schur function expansion of $\tau(t)$ takes the form

$$
\tau(t)=s_{\nu^{(g-1)}}(t)+\text { (higher weight terms). }
$$

By the definition of the frame $Y^{ \pm}, \tau\left(t ; Y^{ \pm}\right)$has the expansion of this form. Thus $\tau(t)=$ $\tau\left(t ; Y^{ \pm}\right)$.

\section{Degeneration of sigma function}

\subsection{Relation of sigma functions}

Expand

$$
\log \left(1-\alpha z^{2}\right)^{-1}=\sum_{j=1}^{\infty}\left(2 \alpha^{j}\right) \frac{z^{2 j}}{2 j}
$$

By (3.12)

$$
\tau(t ; X)=\exp \left(2 \sum_{j=1}^{\infty} \alpha^{j} t_{2 j}\right) \tau\left(t ; \widetilde{X}_{0}\right) .
$$

We denote the limit of $\sigma^{(g)}(u)$ by $\sigma_{g}^{(g) 0}(u)$ etc. by putting the upper index 0 to each quantity. Then, by taking the limit of (4.5) we have

$$
\tau(t ; X)=\exp \left(2 \sum_{j=1}^{\infty} \alpha^{j} t_{2 j}-\sum_{i=1}^{\infty} c_{2 i}^{(g) 0} t_{2 i}+\frac{1}{2} \widehat{q}^{(g) 0}(t)\right) \sigma^{(g) 0}\left(B^{(g) 0} t\right) .
$$


Substitute (4.11) and (5.2) to (3.19). We get

Theorem 5.1. The following relation is valid:

$$
\begin{aligned}
& \sigma^{(g) 0}\left(B^{(g) 0} t\right)=\frac{(-1)^{g-1}}{2 y_{0}} \mathrm{~s}_{0}^{(g-2)} \exp \left(-2 \sum_{j=1}^{\infty} \alpha^{j} t_{2 j}+\sum_{i=1}^{\infty} c_{2 i}^{(g) 0} t_{2 i}-\frac{1}{2} \widehat{q}^{(g) 0}(t)+\frac{1}{2} \widehat{q}^{(g-1)}(t)\right) \\
& \times \sum_{\epsilon= \pm} \epsilon \exp \left(\sum_{j=1}^{\infty} d_{j}^{(g-1, \epsilon)} t_{j}\right) \frac{\sigma^{(g-1)}\left(B^{(g-1)} t-p_{\epsilon}\right)}{\sigma_{A_{0}^{(g-2)}}^{(g-1)}\left(-p_{\epsilon}\right)},
\end{aligned}
$$

where \pm are identified with \pm 1 .

Set

$$
B^{(g) 0} t=\left(u_{1}, u_{3}, \ldots, u_{2 g-1}\right)^{t},
$$

which, in terms of coordinates, is written as

$$
u_{2 i-1}=\sum_{j=1}^{\infty} b_{i, 2 j-1}^{(g) 0} t_{2 j-1} . \quad 1 \leq i \leq g,
$$

Then

Lemma 5.2. The following equation holds,

$$
B^{(g-1)} t=\left(u_{1}-\alpha u_{3}, u_{3}-\alpha u_{5}, \ldots, u_{2 g-3}-\alpha u_{2 g-1}\right) .
$$

Proof. The equation (5.5) is equivalent to

$$
\sum_{j=1}^{\infty} b_{i, 2 j-1}^{(g-1)} t_{2 j-1}=u_{2 i-1}-\alpha u_{2 i+1} .
$$

Let us prove it. We have

$$
\begin{aligned}
\mathrm{d} u_{2 i-1}^{(g) 0} & =\frac{z^{2 i-2} \mathrm{~d} z}{\left(1-\alpha z^{2}\right) F_{0}(z)}=\left(\sum_{j=i}^{\infty} b_{i, 2 j-1}^{(g) 0} z^{2 j-2}\right) \mathrm{d} z \\
\mathrm{~d} u_{2 i-1}^{(g-1)} & =\frac{z^{2 i-2} \mathrm{~d} z}{F_{0}(z)}=\left(\sum_{j=i}^{\infty} b_{i, 2 j-1}^{(g-1)} z^{2 j-2}\right) \mathrm{d} z
\end{aligned}
$$

Therefore, for $1 \leq i \leq g-1$, we have

$$
\mathrm{d} u_{2 i-1}^{(g-1)}=\left(1-\alpha z^{2}\right) \mathrm{d} u_{2 i-1}^{(g) 0},
$$

which is equivalent to

$$
b_{i, 2 j-1}^{(g-1)}=b_{i, 2 j-1}^{(g) 0}-\alpha b_{i, 2 j-3}^{(g) 0},
$$


where we set $b_{i j}^{(g) 0}=0$ if $j \leq 0$. Multiplying $t_{2 j-1}$ and summing up we get

$$
\begin{aligned}
\sum_{j=i}^{\infty} b_{i, 2 j-1}^{(g-1)} t_{2 j-1} & =\sum_{j=i}^{\infty} b_{i, 2 j-1}^{(g) 0} t_{2 j-1}-\alpha \sum_{j=i+1}^{\infty} b_{i, 2 j-3}^{(g) 0} t_{2 j-1} \\
& =u_{2 i-1}-\alpha \sum_{j=i+1}^{\infty} b_{i, 2 j-3}^{(g) 0} t_{2 j-1} .
\end{aligned}
$$

Set

$$
\frac{1}{F(z)}=\frac{1}{\left(1-\alpha z^{2}\right) F_{0}(z)}=\sum_{j=0}^{\infty} \beta_{2 j+1} z^{2 j}
$$

By (5.7) we have

$$
b_{i, 2 j-1}^{(g) 0}=\beta_{2 j-2 i+1},
$$

and

$$
u_{2 i-1}=\sum_{j=i}^{\infty} b_{i, 2 j-1}^{(g) 0} t_{2 j-1}=\sum_{j=i}^{\infty} \beta_{2 j-2 i+1} t_{2 j-1} .
$$

Therefore

$$
\sum_{j=i+1}^{\infty} b_{i, 2 j-3}^{(g) 0} t_{2 j-1}=\sum_{j=i+1}^{\infty} \beta_{2 j-2(i+1)+1} t_{2 j-1}=u_{2 i+1} .
$$

Substituting this to (5.10) we get (5.6).

By (5.4) and (5.5) the arguments of $\sigma^{(g) 0}$ and $\sigma^{(g-1)}$ in Theorem 5.1 is directly connected without the variable $t$. Let us study other parts in the formula (5.3).

\subsection{The expression of $t$ by $u$}

Define

$$
\begin{aligned}
& \Lambda_{n}=\frac{1}{2 n} \sum_{j=1}^{2 g+1} \alpha_{j}^{n}=\frac{1}{2 n}\left(2 \alpha^{2}+\sum_{j=1}^{2 g-1} \alpha_{j}^{n}\right) \\
& \Lambda=\left(\Lambda_{1}, \Lambda_{2}, \Lambda_{3}, \ldots\right)
\end{aligned}
$$

Lemma 5.3. $\beta_{2 j+1}=s_{(j)}(\Lambda), \quad j \geq 0$.

Proof. We have

$$
\begin{aligned}
\frac{1}{F(z)}=\exp (-\log F(z)) & =\exp \left(-\frac{1}{2} \sum_{j=1}^{2 g+1} \log \left(1-\alpha_{j} z^{2}\right)\right) \\
& =\exp \left(\sum_{n=1}^{\infty}\left(\frac{1}{2 n} \sum_{j=1}^{2 g+1} \alpha_{j}^{n}\right) z^{2 n}\right)
\end{aligned}
$$




$$
\begin{aligned}
& =\exp \left(\sum_{n=1}^{\infty} \Lambda_{n} z^{2 n}\right) \\
& =\sum_{n=0}^{\infty} s_{(n)}(\Lambda) z^{2 n}
\end{aligned}
$$

Set $t_{j}=0, j \geq 2 g$ and $t_{2 j-1}=0, j \geq g+1$ in (5.4). Then

$$
\left(\begin{array}{c}
u_{1} \\
u_{3} \\
\vdots \\
u_{2 g-1}
\end{array}\right)=\widetilde{M}\left(\begin{array}{c}
t_{1} \\
t_{3} \\
\vdots \\
t_{2 g-1}
\end{array}\right), \quad \widetilde{M}=\left(\begin{array}{ccccc}
1 & \beta_{3} & \beta_{5} & \ldots & \beta_{2 g-1} \\
0 & 1 & \beta_{3} & \ldots & \beta_{2 g-3} \\
0 & \ddots & \ddots & & \vdots \\
\vdots & & \ddots & \ddots & \vdots \\
0 & \ldots & \ldots & 0 & 1
\end{array}\right) .
$$

Set

$$
M=\widetilde{M}^{-1}
$$

The matrix $M$ can be computed as the cofactor matrix.

Lemma 5.4. We have

$$
\left(\begin{array}{c}
t_{1} \\
t_{3} \\
\vdots \\
t_{2 g-1}
\end{array}\right)=M\left(\begin{array}{c}
u_{1} \\
u_{3} \\
\vdots \\
u_{2 g-1}
\end{array}\right), \quad M=\left(\begin{array}{ccccc}
1 & m_{1,2} & m_{1,3} & \ldots & m_{1, g} \\
0 & 1 & m_{2,3} & \ldots & m_{2, g} \\
0 & \ddots & \ddots & & \vdots \\
\vdots & & \ddots & \ddots & \vdots \\
0 & \ldots & \ldots & 0 & 1
\end{array}\right)
$$

where, for $i<j$,

$$
m_{i j}=(-1)^{i+j} \operatorname{det}\left(\begin{array}{ccccc}
s_{(1)} & s_{(2)} & \ldots & \ldots & s_{(j-i)} \\
1 & s_{(1)} & s_{(2)} & \ldots & s_{(j-i-1)} \\
0 & \ddots & \ddots & & \vdots \\
\vdots & \ddots & \ddots & & \vdots \\
0 & \ldots & \ldots & 1 & s_{(1)}
\end{array}\right), \quad s_{(j)}=s_{(j)}(\Lambda) .
$$

\subsection{Difference of bidifferentials}

Let

$$
Q_{i, j}=-\widehat{q}_{i, j}^{(g) 0}+\widehat{q}_{i, j}^{(g-1)}, \quad Q=\left(Q_{2 i-1,2 j-1}\right)_{1 \leq i, j \leq g},
$$

and

$$
f_{g}(x)=(x-\alpha)^{2} \prod_{j=1}^{2 g-1}\left(x-\alpha_{j}\right)=\sum_{i=0}^{2 g+1} \mu_{4 g+2-2 i} x^{i}
$$




$$
f_{g-1}(x)=\prod_{j=1}^{2 g-1}\left(x-\alpha_{j}\right)=\sum_{i=0}^{2 g-1} \widetilde{\mu}_{4 g-2-2 i} x^{i} .
$$

We set $\widetilde{\mu}_{4 g-2-2 i}=0$ if $i<0$ or $i>2 g-1$. The relation

$$
f_{g}(x)=(x-\alpha)^{2} f_{g-1}(x)
$$

implies

$$
\mu_{4 g+2-2 i}=\widetilde{\mu}_{4 g+2-2 i}-2 \alpha \widetilde{\mu}_{4 g-2 i}+\alpha^{2} \widetilde{\mu}_{4 g-2-2 i} .
$$

Define

$$
\begin{aligned}
H_{g}\left(x_{1}, x_{2}\right) & =\sum_{i=0}^{g}\left(x_{1} x_{2}\right)^{i}\left(2 \mu_{4 g+2-4 i}+\mu_{4 g-4 i}\left(x_{1}+x_{2}\right)\right), \\
H_{g-1}\left(x_{1}, x_{2}\right) & =\sum_{i=0}^{g-1}\left(x_{1} x_{2}\right)^{i}\left(2 \widetilde{\mu}_{4 g-2-4 i}+\widetilde{\mu}_{4 g-4-4 i}\left(x_{1}+x_{2}\right)\right) .
\end{aligned}
$$

By computation we have

\section{Lemma 5.5.}

$$
H_{g}\left(x_{1}, x_{2}\right)=\left(x_{1}-\alpha\right)\left(x_{2}-\alpha\right) H_{g-1}\left(x_{1}, x_{2}\right)+\alpha\left(x_{1}-x_{2}\right)^{2} \sum_{i=0}^{g-1} \widetilde{\mu}_{4 g-4-4 i} x_{1}^{i} x_{2}^{i} .
$$

Set $x_{i}=z_{i}^{-2}$ and

$$
y_{i}^{(g-1)}=z_{i}^{-(2 g-1)} F_{0}(z) \quad y_{i}^{(g)}=\left(x_{i}-\alpha\right) y_{i}^{(g-1)}=z_{i}^{-(2 g+1)} F(z) .
$$

Then

$$
\begin{aligned}
\widehat{\Omega}^{(g) 0}\left(x_{1}, x_{2}\right) & =\left(\frac{1}{2\left(x_{1}-x_{2}\right)^{2}}+\frac{H_{g}\left(x_{1}, x_{2}\right)}{4\left(x_{1}-x_{2}\right)^{2} y_{1}^{(g)} y_{2}^{(g)}}\right) \mathrm{d} x_{1} \mathrm{~d} x_{2}, \\
\widehat{\Omega}^{(g-1)}\left(x_{1}, x_{2}\right) & =\left(\frac{1}{2\left(x_{1}-x_{2}\right)^{2}}+\frac{H_{g-1}\left(x_{1}, x_{2}\right)}{4\left(x_{1}-x_{2}\right)^{2} y_{1}^{(g-1)} y_{2}^{(g-1)}}\right) \mathrm{d} x_{1} \mathrm{~d} x_{2} .
\end{aligned}
$$

By Lemma 5.5

$$
\begin{aligned}
-\widehat{\Omega}^{(g) 0}\left(x_{1}, x_{2}\right)+\widehat{\Omega}^{(g-1)}\left(x_{1}, x_{2}\right) & =-\alpha \frac{\sum_{i=0}^{g-1} \widetilde{\mu}_{4 g-4-4 i} x_{1}^{i} x_{2}^{i}}{4 y_{1}^{(g)} y_{2}^{(g)}} \mathrm{d} x_{1} \mathrm{~d} x_{2} \\
& =-\alpha \frac{\sum_{i=0}^{g-1} \widetilde{\mu}_{4 i} z_{1}^{2 i} z_{2}^{2 i}}{F\left(z_{1}\right) F\left(z_{2}\right)} \mathrm{d} z_{1} \mathrm{~d} z_{2} .
\end{aligned}
$$

We have

$$
\frac{1}{F(z)}=\sum_{n=0}^{\infty} s_{(n)}(\Lambda) z^{2 n}
$$

by (5.11) and Lemma 5.3 .

Compute the expansion of the right hand side of (5.16) using (5.17) and compare the expansion coefficients. Then we get 
Proposition 5.6. For $i, j \geq 0$

$$
Q_{2 i+1,2 j+1}=-\alpha \sum_{k=0}^{g-1} \widetilde{\mu}_{4 k} s_{(i-k)} s_{(j-k)},
$$

where $s_{(j)}=s_{(j)}(\Lambda)$.

Example 5.7. The case of $g=2$.

$$
Q_{1,1}=-\alpha, \quad Q_{1,3}=Q_{3,1}=-\alpha s_{(1)}, \quad Q_{3,3}=-\alpha\left(s_{(1)}^{2}+\widetilde{\mu}_{4}\right),
$$

where

$$
s_{(1)}=\alpha-\frac{1}{2} \widetilde{\mu}_{2}
$$

In this case $m_{1,2}=s_{(1)}$ and

$$
t_{1}=u_{1}-s_{(1)} u_{3}, \quad t_{3}=u_{3} .
$$

Then

$$
Q_{1,1} t_{1}^{2}+2 Q_{1,3} t_{1} t_{3}+Q_{3,3} t_{3}^{2}=-\alpha\left(u_{1}^{2}+\widetilde{\mu}_{4} u_{3}^{2}\right) .
$$

\subsection{Final formula for the degenerate sigma function}

Set

$$
\begin{aligned}
u & =\left(u_{1}, u_{3}, \ldots, u_{2 g-1}\right)^{t}, \\
\widetilde{u} & =\left(u_{1}-\alpha u_{3}, u_{3}-\alpha u_{5}, \ldots, u_{2 g-3}-\alpha u_{2 g-1}\right)^{t} \\
d^{(g-1, \pm)} & =\left(d_{1}^{(g-1, \pm)}, d_{3}^{(g-1, \pm)}, \ldots, d_{2 g-1}^{(g-1, \pm)}\right)^{t} .
\end{aligned}
$$

Since $\widehat{q}^{(g) 0}(t), \widehat{q}^{(g-1)}(t)$ do not contain $t_{2 j}, j \geq 1$, we set $t_{2 j}=0, j \geq 1$ and $t_{2 j-1}=0$, $j \geq g+1$ in (5.3). Then

Theorem 5.8. We have

$$
\begin{aligned}
\sigma^{(g) 0}(u)= & \frac{(-1)^{g-1}}{2 y_{0}} \mathrm{~s}_{0}^{(g-2)} \exp \left(\frac{1}{2} u^{t} M^{t} Q M u\right) \\
& \times \sum_{\epsilon= \pm} \epsilon \exp \left(d^{(g-1, \epsilon) t} M u\right) \frac{\sigma^{(g-1)}\left(\widetilde{u}-p_{\epsilon}\right)}{\sigma_{A_{0}^{(g-2)}}^{(g-1)}\left(-p_{\epsilon}\right)}
\end{aligned}
$$

where $M$ is given by Lemma 5.4, $Q$ is given by (5.14), (5.18) and $d^{(g-1, \epsilon)}$ is given by (5.21) and (4.9).

\section{The case of genus two}

In [4] the degeneration of the genus two hyperelliptic sigma function is studied using the linear differential equations satisfied by the sigma function. In this section we derive their formula by our approach. 


\subsection{Problem and the strategy}

Let $\alpha_{1}, \ldots, \alpha_{5}$ be mutually distinct complex numbers, $a$ a complex number and

$$
\begin{array}{ll}
C_{1}: w^{2}=\prod_{j=1}^{5}\left(v-\alpha_{j}\right)=\sum_{j=0}^{5} \mu_{10-2 j} v^{j}, & \mu_{0}=1, \mu_{2}=0, \\
C_{2}: y^{2}=\prod_{j=1}^{5}\left(x-\widetilde{\alpha}_{j}\right)=\sum_{j=0}^{5} \widetilde{\mu}_{10-2 j} x^{j}, \quad \widetilde{\alpha}_{j}=\alpha_{j}+\frac{2}{3} \alpha,
\end{array}
$$

genus two hyperelliptic curves. Two curves $C_{1}$ and $C_{2}$ are isomorphic by

$$
(x, y) \longrightarrow(v, w)=\left(x-\frac{2}{3} \alpha, y\right) .
$$
$C_{1}$ to

Assume that $\alpha \neq \alpha_{j}, j=1,2,3$ and consider the degeneration $\alpha_{4}, \alpha_{5} \rightarrow \alpha$ of the curve

$$
w^{2}=(v-\alpha)^{2} \prod_{j=1}^{3}\left(v-\alpha_{j}\right)=\sum_{j=0}^{5} \mu_{10-2 j}^{0} v^{j} .
$$

Correspondingly the equation (6.2) degenerates to

$$
y^{2}=\left(x-\frac{5}{3} \alpha\right)^{2}\left(x^{3}-\frac{g_{2}}{4} x-\frac{g_{3}}{4}\right)
$$

where we set

$$
g_{2}=-4\left(\widetilde{\alpha}_{1} \widetilde{\alpha}_{2}+\widetilde{\alpha}_{1} \widetilde{\alpha}_{3}+\widetilde{\alpha}_{2} \widetilde{\alpha}_{3}\right), \quad g_{3}=4 \widetilde{\alpha}_{1} \widetilde{\alpha}_{2} \widetilde{\alpha}_{3} .
$$

Notice that the coefficient of $x^{2}$ becomes zero in the second factor of the right hand side of (6.5). This is the reason why we consider the shift by $(2 / 3) \alpha$ in (6.2). Notice that, due to $\mu_{2}=0$, the coefficients of $\prod_{j=1}^{3}\left(t-\alpha_{j}\right)$ in (6.4) becomes $2 \alpha$ which is not zero if $\alpha \neq 0$.

In [4] the limit of the sigma function of $C_{1}$ is expressed by the sigma function of the elliptic curve

$$
C_{3}: y^{2}=x^{3}-\frac{g_{2}}{4} x-\frac{g_{3}}{4} .
$$

Although $C_{1}$ and $C_{2}$ are isomorphic, their sigma functions are not the same (see Remark 6.2). So we can not apply our result directly to the case studied in [4]. However the idea to use tau function can be applied. The strategy is as follows. We first show that the tau functions of $C_{1}$ and $C_{2}$ are the same. By Theorem 3.2 the limit of the tau function of $C_{2}$ is expressed by the tau functions of $C_{1}$. We can not apply Theorem 4.4 to the tau functions of $C_{1}$, since the sigma function formula of the tau function depends on the choice of a local coordinate. As we shall see, for $C_{1}$, we need to use the local coordinate $z$ at $\infty$ given by

$$
v=\frac{1}{z^{2}}-\frac{2}{3} \alpha
$$

While Theorem 4.4 is derived using the local coordinate $z$ such that

$$
v=\frac{1}{z^{2}} \text {. }
$$

However it is possible to make the sigma function formula of the tau function using the local coordinate satisfying (6.8) in a completely similar way to the case of (6.9). 


\subsection{Tau function of $C_{1}$ and $C_{2}$}

Take the local coordinate $z$ at $\infty$ of $C_{2}$ by

$$
x=\frac{1}{z^{2}}, \quad y=\frac{1}{z^{5}}(1+O(z)) .
$$

By (6.3) $z$ becomes a local coordinate at $\infty$ of $C_{1}$ such that

$$
v=\frac{1}{z^{2}}-\frac{2}{3} \alpha, \quad w=\frac{1}{z^{5}}(1+O(z)) .
$$

By (3.3) a basis of $H^{0}\left(C_{2}, \mathcal{O}(* \infty)\right)$ is given by

$$
z^{-2 i}, \quad z^{-5-2 i} F(z), \quad i \geq 0,
$$

where

$$
F(z)=\left(\prod_{j=1}^{5}\left(1-\widetilde{\alpha}_{j} z^{2}\right)\right)^{\frac{1}{2}}
$$

A basis of $H^{0}\left(C_{1}, \mathcal{O}(* \infty)\right)$ is given by

$$
v^{i}, \quad v^{i} w, \quad, i \geq 0
$$

which, in terms of $z$, is written as

$$
\left(z^{-2}-\frac{2}{3} \alpha\right)^{i}, \quad z^{-5}\left(z^{-2}-\frac{2}{3} \alpha\right)^{i} F(z), \quad i \geq 0 .
$$

We easily have

Lemma 6.1. The vector space generated by (6.11) and that by (6.12) coincide.

By Lemma 6.1 $C_{1}$ and $C_{2}$ with the local coordinate $z$ determine the same frame $\widetilde{X}$ given by (3.5). Therefore their tau functions are the same. Consequently Theorem 3.2 holds for tau functions of $C_{1}$ and $C_{3}$.

\section{3 $\tau(t ; X)$ by sigma function of $C_{1}$}

Redefine $b_{i, j}^{(g)}, \widehat{q}_{i, j}^{(g)}, c_{i}^{(g)}$ by the expansion coefficients in terms of the local coordinate $z$ of (6.10). Then it can be proved that Theorem 4.4 holds without any change.

Let $\wp(u)$ be the Weierstrass elliptic function. The points of $C_{3}$ are parametrized as

$$
(x, y)=\left(\wp(u), \frac{1}{2} \wp^{\prime}(u)\right), \quad u \in \mathbb{C} .
$$

By definition

$$
p_{ \pm}=\left(\frac{5}{3} \alpha, \pm y_{0}\right) \in C_{3}
$$


We choose $a \in \mathbb{C}$ such that

$$
p_{ \pm}=\left(\wp(a), \frac{1}{2} \wp^{\prime}( \pm a)\right)
$$

which means

$$
\alpha=\frac{3}{5} \wp(a), \quad y_{0}=\frac{1}{2} \wp^{\prime}(a) .
$$

For the curve $C_{3}$

$$
\mathrm{d} u_{1}^{(1)}=-\frac{\mathrm{d} x}{2 y}
$$

Then

$$
\int_{\infty}^{p_{ \pm}} \mathrm{d} u_{1}^{(1)}=-\int_{\infty}^{p_{ \pm}} \frac{\mathrm{d} x}{2 y}=-( \pm a)=\mp a
$$

In the following everything is computed in the limit $\alpha_{4}, \alpha_{5} \rightarrow \alpha$. We have

$$
\begin{aligned}
\mathrm{d} u_{1}^{(2) 0} & =-\frac{v \mathrm{~d} v}{2 w}=\left(1+\alpha z+O\left(z^{4}\right)\right) d z \\
\mathrm{~d} u_{3}^{(2) 0} & =-\frac{\mathrm{d} v}{2 w}=\left(z^{2}+O\left(z^{4}\right)\right) d z
\end{aligned}
$$

Thus

$$
B^{(2) 0}=\left[\begin{array}{cccccc}
1 & 0 & \alpha & 0 & * & \ldots \\
0 & 0 & 1 & 0 & * & \ldots
\end{array}\right]
$$

By definition

$$
\begin{aligned}
\widehat{\Omega}^{(2) 0}\left(p_{1}, p_{2}\right) & =\frac{2 w_{1} w_{2}+\sum_{i=0}^{2} v_{1}^{i} v_{2}^{i}\left(2 \mu_{10-4 i}^{(2) 0}+\mu_{8-4 i}^{(2) 0}\left(v_{1}+v_{2}\right)\right)}{4\left(v_{1}-v_{2}\right)^{2} w_{1} w_{2}} \mathrm{~d} v_{1} \mathrm{~d} v_{2} \\
& =\left(\frac{1}{\left(z_{1}-z_{2}\right)^{2}}+\sum_{i, j \geq 1} \widehat{q}_{2 i-1,2 j-1}^{(2) 0} z_{1}^{2(i-1)} z_{2}^{2(i-1)}\right) \mathrm{d} z_{1} \mathrm{~d} z_{2} .
\end{aligned}
$$

Then

$$
\begin{aligned}
& \widehat{q}_{1,1}^{(2) 0}=\frac{1}{5} \wp(a), \quad \widehat{q}_{1,3}^{(2) 0}=\widehat{q}_{3,1}^{(2) 0}=\frac{9}{25} \wp(a)^{2}+\frac{g_{3}}{8}, \\
& \widehat{q}_{3,3}^{(2) 0}=-\frac{3 g_{2}}{20} \wp(a)+\frac{3 g_{3}}{8}+\frac{63}{125} \wp(a)^{3} .
\end{aligned}
$$

Similarly to (4.4) $c_{2 i-1}^{(2) 0}=0, i \geq 1$. If

$$
t_{2 j}=t_{2 j+3}=0, \quad j \geq 1,
$$

we have

$$
B^{(2) 0} t=\left[\begin{array}{c}
t_{1}+\alpha t_{3} \\
t_{3}
\end{array}\right],
$$




$$
\widehat{q}^{(2) 0}(t)=\sum_{i, j=1}^{2} \widehat{q}_{2 i-1,2 j-1}^{(2) 0} t_{2 i-1} t_{2 j-1} .
$$

Therefore, taking (5.1) into account, we have

$$
\tau\left(\left(t_{1}, 0, t_{3}, 0, \ldots\right)^{t} ; X\right)=\exp \left(\frac{1}{2} \widehat{q}^{(2) 0}(t)\right) \sigma^{(2) 0}\left(t_{1}+\alpha t_{3}, t_{3}\right) .
$$

\subsection{Tau function $\tau\left(t ; Y^{ \pm}\right)$by sigma function of $C_{3}$}

Put $g=2$ in Theorem 4.9 and use $s_{0}^{(0)}=1, A_{0}^{(0)}=\emptyset$. Then

$$
\begin{aligned}
\tau\left(\left(t_{1}, 0, t_{3}, 0, \ldots\right)^{t} ; Y^{ \pm}\right) \\
\quad=\exp \left(\sum_{j=1,3} d_{j}^{(1, \pm)} t_{j}+\frac{1}{2} \widehat{q}^{(1)}(t)\right) \frac{\sigma\left(B^{(1)} t-p_{ \pm}\right)}{\sigma\left(-p_{ \pm}\right)}
\end{aligned}
$$

where $\sigma(u)=\sigma^{(1)}(u)$ is the Weierstrass sigma function.

We have

$$
\mathrm{d} u_{1}^{(1)}=-\frac{\mathrm{d} x}{2 y}=\left(1+O\left(z^{4}\right)\right) \mathrm{d} z
$$

and

$$
\begin{aligned}
& B^{(1)}=[1,0,0,0, *, \ldots] . \\
& B^{(1)} t=t_{1} .
\end{aligned}
$$

For the bidifferential of $C_{3}$ we have

$$
\begin{aligned}
& \widehat{\Omega}^{(1)}\left(p_{1}, p_{2}\right) \\
= & \frac{2 y_{1} y_{2}+x_{1} x_{2}\left(x_{1}+x_{2}\right)-\frac{1}{4} g_{2}\left(x_{1}+x_{2}\right)-\frac{1}{2} g_{3}}{4\left(x_{1}-x_{2}\right)^{2} y_{1} y_{2}} \mathrm{~d} x_{1} \mathrm{~d} x_{2} \\
= & \left(\frac{1}{\left(z_{1}-z_{2}\right)^{2}}+\frac{g_{2}}{8}\left(z_{1}^{2}+z_{2}^{2}\right)+\frac{g_{3}}{8}\left(z_{1}^{4}+z_{2}^{4}+3 z_{1}^{2} z_{2}^{2}\right)+\cdots\right) \mathrm{d} z_{1} \mathrm{~d} z_{2} .
\end{aligned}
$$

Therefore

$$
\widehat{q}_{1,1}^{(1)}=0, \quad \widehat{q}_{1,3}^{(1)}=\widehat{q}_{3,1}^{(1)}=\frac{g_{2}}{8}, \quad \widehat{q}_{3,3}^{(1)}=\frac{3 g_{3}}{8} .
$$

By (4.9) and (6.15)

$$
\log \left(\frac{\sigma( \pm a)}{\sigma(p \pm a)}\right)-\frac{1}{2} \widehat{q}^{(1)}([z] \mid[z])=\sum_{j=1}^{\infty} d_{j}^{(1, \pm)} \frac{z^{j}}{j}
$$

Since $\widehat{q}^{(1)}([z] \mid[z])$ contains only even powers of $z, d_{j}^{(1, \pm)}, j=1,3$ are the expansion coefficients of the first term of the left hand side of (6.25). By computation we have

$$
d_{1}^{(1, \pm)}=\mp \zeta(a), \quad d_{3}^{(1, \pm)}= \pm \frac{1}{2} \wp^{\prime}(a),
$$


where $\zeta(u)$ is the Weierstrass zeta function. Substituting (6.23), (6.26) and (6.15) into (6.22) we have

$$
\begin{aligned}
\tau\left(\left(t_{1}, 0, t_{3}, 0, \ldots\right)^{t} ; Y^{ \pm}\right) \\
\\
\quad=\exp \left(\mp \zeta(a) t_{1} \pm \frac{1}{2} \wp^{\prime}(a) t_{3}+\frac{1}{2} \widehat{q}^{(1)}(t)\right) \frac{\sigma\left(t_{1} \pm a\right)}{\sigma( \pm a)} .
\end{aligned}
$$

\subsection{Genus two degenerate formula}

Substitute (6.21) and (6.27) into (3.19) in Theorem 3.2, use (6.14) and solve in $\sigma^{(2) 0}$. Then we have

$$
\begin{aligned}
& \sigma^{(2) 0}\left(t_{1}+\alpha t_{3}, t_{3}\right) \\
= & \frac{-1}{\wp^{\prime}(a)} \exp \left(\frac{1}{2}\left(-q^{(2) 0}(t)+q^{(1)}(t)\right)\right) \\
& \times \sum_{\epsilon= \pm 1} \epsilon \exp \left(-\epsilon \zeta(a) t_{1}+\epsilon \frac{1}{2} \wp^{\prime}(a) t_{3}\right) \frac{\sigma\left(t_{1}+\epsilon a\right)}{\sigma(\epsilon a)} .
\end{aligned}
$$

Set

$$
u_{1}=t_{1}+\alpha t_{3} . \quad u_{3}=t_{3}
$$

Solving in $x$ we have

$$
t_{1}=u_{1}-\alpha u_{3}, \quad t_{3}=u_{3}
$$

Then

$$
\begin{aligned}
\frac{1}{2}\left(-q^{(2) 0}(t)+q^{(1)}(t)\right)= & -\frac{1}{10} \wp(a) u_{1}^{2}-\frac{6}{25} \wp(a)^{2} u_{1} u_{3} \\
& +\left(-\frac{9}{125} \wp(a)^{3}+\frac{3}{40} g_{2} \wp(a)\right) u_{3}^{2}, \\
-\zeta(a) t_{1}+\frac{1}{2} \wp(a) t_{3}= & -\zeta(a) u_{1}+\left(\frac{3}{5} \zeta(a) \wp(a)+\frac{1}{2} \wp^{\prime}(a)\right) u_{3} .
\end{aligned}
$$

Substitute (6.30), (6.31), (6.32) into (6.28) we get, with $u=\left(u_{1}, u_{3}\right)^{t}$,

$$
\begin{aligned}
\sigma^{(2) 0}(u)= & \frac{-1}{\sigma(a) \wp^{\prime}(a)} \exp \left(-\frac{3}{5} \wp(a)\left(\frac{1}{6} u_{1}^{2}+\frac{2}{5} \wp(a) u_{1} u_{3}+\left(\frac{3}{25} \wp(a)^{2}-\frac{g_{2}}{8}\right) u_{3}^{2}\right)\right) \\
& \times \sum_{\epsilon= \pm 1} \exp \left(\epsilon\left(-\zeta(a) u_{1}+\left(\frac{3}{5} \zeta(a) \wp(a)+\frac{1}{2} \wp^{\prime}(a)\right) u_{3}\right)\right) \\
& \times \sigma\left(u_{1}-\frac{3}{5} \wp(a) u_{3}+\epsilon a\right),
\end{aligned}
$$

which coincides with the formula in Theorem 1 of [4] up to sign. The discrepancy of the sign is due to the different normalization of the sigma function. 
Remark 6.2. Recall that the left hand side of (6.33) is the degenerate sigma function of the curve $C_{1}$. The formula of Theorem 5.8 gives a formula for the degenerate sigma function of the curve $C_{2}$. By Example 5.7 it is obtained in (6.33) by replacing the exponent of the exponential in front of the sum symbol by

$$
-\frac{5}{6} \alpha\left(u_{1}^{2}+\widetilde{\mu}_{4} u_{3}^{2}\right)
$$

It shows that the sigma functions of $C_{1}$ and $C_{2}$ do not coincide.

\section{Acknowledegements}

This work was supported by the JSPS Grants-in-Aid for Scientific Research No. 15K04907.

\section{References}

[1] S. Abenda and P. Grinevich, Rational degenerations of M-curves, totally positive Grassmannians and KP-solitons, Comm. Math. Phys. 361 (2018) 1029-1081, https://doi:10.1007/s00220-018-3123-y.

[2] T. Ayano and A. Nakayashiki, On Addition Formulae for Sigma Functions of Telescopic Curves, Symmetry, Integrability and Geometry: Methods and Applications (SIGMA) 9 (2013), 046, 14 pages.

[3] H. F. Baker, Abelian functions. Abels theorem and the allied theory of theta functions, 1898 Cambridge Mathematical Library, Cambridge University Press, Cambridge, 1995.

[4] J. Bernatska and D. Leykin, On degenerate sigma-functions in genus two, Glasgow Math. J (2018), arXiv:1509.01490.

[5] V. M. Buchstaber, V. Z. Enolski and D. V. Leykin, Kleinian functions, hyperelliptic Jacobians and applications, in Reviews in Math. and Math. Phys. 10, No.2, Gordon and Breach, London, 1997, 1-125.

[6] V. M. Buchstaber, V. Z. Enolski and D. V. Leykin, Multi-Dimensional SigmaFunctions, arXiv:1208.0990, 2012

[7] V. M. Buchstaber and D. V. Leykin, Addition Laws on Jacobian Varieties of Plane Algebraic Curves. Proceedings of the Steklov Institute of Mathematics, 251 (2005), pp. 172. Translated from Trudy Matematicheskogo Instituta imeni V.A. Steklova, 251, (2005), pp. 54126.

[8] V. M. Buchstaber, D. V. Leykin and V. Z. Enolski, Uniformization of Jacobi Varieties of Trigonal Curves and Nonlinear Differential Equations. Funct.Anal.Appl. (2000) 34, Issue $3,159-171$ 
[9] E. Date, M. Kashiwara, M. Jimbo, and T. Miwa, Transformation groups for soliton equations, in "Nonlinear Integrable Systems - Classical Theory and Quantum Theory", M. Jimbo and T. Miwa (eds.), World Sci., Singapore, 1983, pp.39-119.

[10] J. C. Eilbeck, V. Z. Enolski and J. Gibbons, Sigma, tau and Abelian functions of algebraic curves, J. Phys. A: Math. Theor. 43 (2010), 455216.

[11] J. C. Eilbeck, V. Z. Enolski, S. Matsutani, Y. Ônishi and E. Previato, Abelian functions for trigonal curves of genus of genus three, Int. Math. Res. Not. 2007, (2007): rnm 140-68.

[12] V. Enolski and J. Harnad, Schur function expansions of KP tau functions associated with algebraic curves. (Russian) Uspekhi Mat. Nauk 66 (2011), no. 4(400), 137-178; translation in Russian Math. Surveys 66 (2011), no. 4, 767-807.

[13] B. Eynard, Notes about a combinatorial expression of the fundamental second kind differential on an algebraic curve, arXiv:1805.07247

[14] J. Fay, Theta-Functions on Riemann Surfaces, Springer Lecture Notes in Mathematics 352, 1973.

[15] I. G. Macdonald, Symmetric Functions and Hall Polynomials, second edition, Oxford University Press, 1995.

[16] Y. Kodama, KP solitons and the Grassmannians, Springer, 2017.

[17] I.M. Krichever, Methods of algebraic geometry in the theory of nonlinear equations, Russ. Math. Surv., Vol. 32 (1977), 185-213

[18] S. Matsutani and E. Previato, Jacobi inversion on strata of the Jacobian of the $C_{r s}$ curve $y^{r}=f(x)$, I,II J. Math. Soc. Japan, 60, No. 4 (2008), 1009-1044. 66, No. 2 (2014), 647-692.

[19] D. Mumford, Tata lectures on theta II, Birkhauser, 1983.

[20] A. Nakayashiki, On algebraic expressions of sigma functions for $(n, s)$ curves, Asian J. Math. 14 (2010), 175211.

[21] A. Nakayashiki, Sigma function as a tau function, Int. Math. Res. Not. IMRN 2010-3 (2010), 373-394.

[22] A. Nakayashiki, Tau function approach to theta functions, Int. Math. Res. Not. IMRN 2016-17 (2016), 5202-5248.

[23] A. Nakayashiki, Degeneration of trigonal curves and solutions of the KP-hierarchy, Nonlinearity 31 (2018), 3567-3590.

[24] A. Nakayashiki, On reducible degeneration of hyperelliptic curves and soliton solutions, arXiv:1808.06748. 
[25] A. Nakayashiki and K. Yori, Derivatives of Schur, tau and sigma functions, on AbelJacobi images, in Symmetries, Integrable Systems and Representations, K.Iohara, S. Morier-Genoud, B. Remy (eds.), Springer, 2012, 429-462.

[26] Y. Onishi, Determinant expressions for hyperelliptic functions, with an Appendix by Shigeki Matsutani: Connection of the formula of Cantor and Brioschi-Kiepert type, Proc. Edinburgh Math. Soc. 48 (2005), 705-742.

[27] M. Sato and Y. Sato, Soliton equations as dynamical systems on infinite dimensional Grassmann manifold, in "Nolinear Partial Differential Equations in Applied Sciences", P. D. Lax, H. Fujita and G. Strang (eds.), North-Holland, Amsterdam, and Kinokuniya, Tokyo, 1982, pp.259-271.

[28] J. Suzuki, Klein's Fundamental 2-Form of Second Kind for the $C_{a b}$ Curves Symmetry, Integrability and Geometry: Methods and Applications (SIGMA) 13 (2017), 017, 13 pages

[29] G. Segal and G. Wilson, Loop groups and equations of KdV type, Publ. Math. IHES 61 (1985) 5-65. 


\title{
Sato Grassmannian and Degenerate Sigma Function
}

\author{
Julia Bernatska* $^{*}$ Victor Enolski ${ }^{\dagger}$, and Atsushi Nakayashiki ${ }^{\ddagger}$
}

Dedicated to Mikio Sato on his 90th birthday

\begin{abstract}
The degeneration of the hyperelliptic sigma function is studied. We use the Sato Grassmannian for this purpose. A simple decomposition of a rational function gives a decomposition of Plücker coordinates of a frame of the Sato Grassmannian. It then gives a decomposition of the tau function corresponding to the degeneration of a hyperelliptic curve of genus $g$ in terms of the tau functions corresponding to a hyperelliptic curve of genus $g-1$. Since the tau functions are described by sigma functions, we get the corresponding formula for the degenerate hyperelliptic sigma function.
\end{abstract}

\section{Introduction}

We study an algebraic curve of genus $g>1$ and the associated sigma-function of $g$ variables which represents a natural generalization of the Weierstrass elliptic sigma function to curves with higher genus [7, 9, 26, 36]. Although this multi-variate sigma function is defined as a certain modification of the Riemann theta function (see Definition 4.6), it has several nice properties which are absent in Riemann's theta function. The most important one is the modular invariance which means that it is invariant under the change of a canonical homology basis defining period matrices. For some class of algebraic curves such as $(n, s)$-curves a more strong property holds. Namely the sigma function can be expanded to a power series whose coefficients are polynomials of the coefficients of the defining equation of the curve [34, 35, 2]. This property, in particular, implies that the sigma function has a well defined limit according as the limit of the defining equation of the curve. Consequently the study of the limits of the sigma functions appears as an interesting and challenging problem. We remark that the period matrix defining the Riemann theta function diverges in general when a curve degenerates to a singular curve and the setting of the degeneration problem itself is non-trivial in this case [5, 18].

The degeneration problem also attracts much attention in the study of integrable systems. Solutions of KP type equations are built of the sigma functions by known rules

\footnotetext{
*National University of Kyiv-Mohyla Academy, H. Skovorody St. 2, 04655 Kyiv, Ukraine, jbernatska@gmail.com

${ }^{\dagger}$ National University of Kyiv-Mohyla Academy, H. Skovorody St. 2, 04655 Kyiv, Ukraine, venolski@googlemail.com

${ }^{\ddagger}$ Department of Mathematics, Tsuda University, Kodaira, Tokyo 187-8577, Japan, atsushi@tsuda.ac.jp
} 
[30, 14, 35]. In particular they can be given as logarithmic derivatives of the sigmafunction. Degenerate solutions appear when the curves have multiple points, in particular, double points. A degenerate genus $g$ solution may be understood as a soliton on a finitegap background expressed in terms of the hyperbolic function and the sigma function (see Chapter 4 of [5] for the genus one background). Such solutions are in great demands of applied sciences and explicit formulae in closed form are requested (see [22, 21, 20] and references therein).

In this paper we study the degeneration of the sigma function when a hyperelliptic curve of genus $g$ degenerates to a curve of genus $g-1$ with a double point. To this end we study the degenerations of tau functions of the KP-hierarchy. By using the Sato theory on the KP-hierarchy [41] the problem on tau functions reduces to a more easy problem on the frames of the Sato Grassmannian. In this way we first derive the formula of the degenerate tau function. Since the tau functions are expressed by the sigma functions, the formula for the degenerate sigma function follows from that for the tau function. This is the main idea of this paper.

Here we deal with the case of hyperelliptic curves. But the method first manifested in [37] on the genus zero degeneration of trigonal curves has a wider area of applicability. Thus we consider the higher genus degeneration of hyperelliptic curve as a good laboratory for further generalizations. We explain the main features of the method in more detail in the following.

First we show that the Sato theory, commonly considered as a deep theoretical description of integrability, can be implemented for the derivation of explicit formulae in a very concrete problem - the construction of degenerate solutions in an explicit and closed form. In the case considered the hyperellptic curve with a double point leads to the decomposition of the Grassmannian frame and the decomposition of the tau function of genus $g$ to the tau functions of genus $g-1$ (Theorem 3.2).

Next, we incorporate the viewpoint of [7] and further developments, in particular, [40, 11, 15, 34, 39, 36, 9, 31, 3, 10, that the sigma function provides an algebraic counterpart of the Riemann theta function and an effective language to deal with the algebro-geometric integrability of soliton equations. After [35, 16, 14, the tau function is expressed in terms of the sigma function and the interrelation of sigma and tau is a part of the construction. To derive the sigma function formula of the tau function corresponding precisely to a point of the Sato Grassmannian some detailed consideration is necessary. For some tau function which appears in the relation in Theorem 3.2 the formula is already known [35] (see Theorem 4.101). We derive the sigma function formula for the remaining tau functions in the relation (see Theorem 4.18).

Also our derivation is based on the notion of Schottky-Klein bidifferential whose fundamental role in the construction of Abelian function associated with Riemann surfaces was elucidated in [18]. Later, in [43] a remarkable formula for the tau function corresponding to points of a Grassmannian associated with algebro-geometric data was discovered. It is expressed by the Riemann theta function and the normalized bidifferential.

In case a point of the Grassmannian is given algebraically in terms of the defining equation of an algebraic curve, it is more natural to realize the tau function in algebraic form using the sigma function. In such expression the normalized bidifferential is replaced by a non-normalized algebraic bidifferential which is expressed in terms of curve coordinates [35, 14. For hyperelliptic curves such representation of the bidifferential is known 
from H.Baker's monograph 4 and nowadays it is obtained for wider classes of curves [12, 34, 42, 17]. As a consequence of this rewriting of the tau function, the sigma function is conversely expressed using the tau function and algebraic quantities. We use it to resolve the posted degeneration problem.

The derivation of the formula for the degenerate sigma function finally reduces to calculating the difference of bidifferentials corresponding to the degenerate limit of a genus $g$ curve and the non-singular curve of genus $g-1$. We demonstrate that, with the help of the explicit algebraic expression of the bidifferential, it can neatly be expressed in terms of the coefficients of the defining equation of the curve. In this way we get the formula which expresses the degenerate sigma function of genus $g$ in terms of the sigma functions of genus $g-1$ (Theorem [5.8). The case of genus two was previously studied in [6], where the system of linear differential equations satisfied by the sigma function [11] was used to derive the formula. Our result generalizes it to the case of genus $g$ by another method (see section 6 for details).

Here it should be emphasized that the method proposed here permits to avoid calculations of both limits of the Riemann matrix and limits of second kind transcendents entering to the definition of sigma. Then the whole procedure reduces to routine expansions of algebraic quantities such as the non-normalized bidifferential represented in algebraic form. Thus we can expect that it is effective for the more complicated curves than hyperelliptic curves.

Recently degenerations of curves to genus $g=0$ and associated trigonometric solutions of the KP hierarchies were studied in [29, 1] (see also references therein). It is shown that the Sato Grassmannian approach is effective in this case [37, 38. We believe that our consideration will permit us to extend interesting results and observation found in these publications to the case of genus $g>1$ degeneration of a curve.

The organization of the paper is as follows. In section 2 we recall the definition of the Sato Grassmannian, which we denote by UGM, and the correspondence between solutions (tau functions) of the KP-hierarchy and points of UGM. We define points of UGM from some spaces of functions on hyperelliptic curves and consider the corresponding tau functions in section 3 . We introduce frames $\widetilde{X}, X, Y^{ \pm}$. The frame $\widetilde{X}$ is associated with a non-singular curve of genus $g$ and $X$ is its degenerate limit. The frames $Y^{ \pm}$correspond to the curve of genus $g-1$. By examining the description of frames we derive the expression of the tau function of $X$ as a linear combination of the tau functions of $Y^{ \pm}$. In section 4 we first recall the definition of the fundamental sigma function, which is simply called the sigma function in the other part of the paper, and the sigma function expression of the tau function of $\widetilde{X}$ given in [35. Then we derive the formulas of the tau functions for $Y^{ \pm}$in terms of the sigma function. For the derivation we use several properties of the sigma function [40, 39, 36]. By substituting the sigma function formulas of tau functions to the relation derived in section 3 , the formula for the degenerate sigma function of genus $g$ in terms of the sigma function of genus $g-1$ is derived in section 5 . The variables of the tau function have a universal character which means that they do not depend on the genus of curves. However the variables of the sigma function depend on the genus of the curve. To connect the variables of the sigma functions of different genus and to express the quantities in the formula for the degenerate sigma functions we examine the expansions of the holomorphic differentials and the meromorphic bidifferentials. In section 6 , we show that the formula of [6] for the degenerate sigma function of genus two can be derived by 
the method proposed in this paper.

\section{Sato Grassmannian and tau function}

In this section we recall the definition of the Sato Grassmannian, which we denote by UGM (universal Grassmann manifold) after M.Sato, and the correspondence between solutions (tau functions) of the KP-hierarchy and points of UGM [41].

In the following we denote by $\mathbb{Z}$ the set of integers and by $\mathbb{N}$ the set of positive integers. Let

$$
V=\mathbb{C}((z))=\left\{\sum_{-\infty \ll i<+\infty} a_{i} z^{i} \mid a_{i} \in \mathbb{C}\right\}
$$

be the vector space of Laurent series in $z$ and

$$
V_{\emptyset}=\mathbb{C}\left[z^{-1}\right], \quad V_{0}=z \mathbb{C}[[z]] .
$$

Then

$$
V=V_{\emptyset} \oplus V_{0} .
$$

Define the projection map from $V$ to $V_{\emptyset}$ by $\pi$ :

$$
\pi: V \rightarrow V / V_{0} \simeq V_{\emptyset}
$$

The Sato Grassmannian UGM is defined as the set of subspaces $U$ of $V$ such that $\operatorname{Ker}\left(\left.\pi\right|_{U}\right)$ and $\operatorname{Coker}\left(\left.\pi\right|_{U}\right)$ are finite dimensional and their dimensions are equal.

Example 2.1. $U=V_{\emptyset} \in$ UGM. In this case $\operatorname{dim} \operatorname{Ker}(\pi \mid U)=\operatorname{dim} \operatorname{Coker}(\pi \mid U)=0$.

Example 2.2. $U=\mathbb{C} z+\sum_{i=0}^{\infty} \mathbb{C} z \wp^{(i)}(z) \in \mathrm{UGM}$, where $\wp(z)$ is the Weierstrass elliptic function and $\wp^{(i)}(z)=\mathrm{d}^{i} \wp(z) / \mathrm{d} z^{i}$. In this case $\operatorname{dim} \operatorname{Ker}(\pi \mid U)=\operatorname{dim} \operatorname{Coker}(\pi \mid U)=1$.

We set

$$
e_{i}=z^{i+1}
$$

A basis $\xi=\left(\ldots, v_{3}, v_{2}, v_{1}\right)$ of $U \in \mathrm{UGM}$ is called a frame of $U$. Write

$$
v_{j}=\sum_{-\infty \ll i<+\infty} \xi_{i, j} e_{i}
$$

and identify $v_{j}$ with the column vector $\left(\xi_{i, j}\right)_{i \in \mathbb{Z}}$,

$$
v_{j}=\left[\begin{array}{c}
\vdots \\
\xi_{-1, j} \\
\xi_{0, j} \\
\xi_{1, j} \\
\vdots
\end{array}\right] .
$$


Then $\xi$ can be expressed as a $\mathbb{Z} \times \mathbb{N}$ matrix $\left(\xi_{i, j}\right)_{i \in \mathbb{Z}, j \in \mathbb{N}}$.

For any point $U$ of UGM it is always possible to take a frame in the following form: there exists $l \geq 1$ such that, for $j \geq l$,

$$
v_{j}=e_{-j}+\sum_{i>-j} \xi_{i, j} e_{i}
$$

In the matrix form it means that the frame has the following form

$$
\xi=\left[\begin{array}{cccccc}
\ddots & O & \vdots & \vdots & & \vdots \\
\cdots & 1 & 0 & * & \cdots & * \\
\cdots & * & 1 & * & \cdots & * \\
\vdots & \vdots & \vdots & \vdots & & \vdots
\end{array}\right]
$$

where the right most 1 in $(2.2)$ is the $(-l, l)$ component. In the following we assume that a frame is always of this form.

A sequence of integers $M=\left(m_{1}, m_{2}, \ldots\right)$ such that

$$
\begin{aligned}
& m_{1}>m_{2}>\cdots, \\
& m_{j}=-j \quad \forall j>>0,
\end{aligned}
$$

is called a Maya diagram. The set of Maya diagrams and that of partitions bijectively correspond to each other by

$$
\begin{aligned}
M=\left(m_{1}, m_{2}, \ldots\right) & \longrightarrow \lambda(M)=\left(m_{1}+1, m_{2}+2, \ldots\right), \\
\lambda=\left(\lambda_{1}, \lambda_{2}, \ldots\right) & \longrightarrow M(\lambda)=\left(\lambda_{1}-1, \lambda_{2}-2, \ldots\right) .
\end{aligned}
$$

For a partition $\lambda$ the Plücker coordinate $\xi_{\lambda}$ of a frame $\xi$ is defined by

$$
\begin{aligned}
& \xi_{\lambda}=\operatorname{det}\left(\xi_{m_{i}, j}\right)_{i, j \in \mathbb{N}}, \\
& M(\lambda)=\left(m_{1}, m_{2}, \ldots\right) .
\end{aligned}
$$

The infinite determinant is well defined for a frame of the form (2.2).

The Schur function $s_{(i)}(t), t=\left(t_{1}, t_{2}, \ldots\right)^{t}$, corresponding to the partition $(i), i \geq 0$, is defined by

$$
\exp \left(\sum_{i=1}^{\infty} t_{i} k^{i}\right)=\sum_{i=0}^{\infty} s_{(i)}(t) k^{i} .
$$

For an arbitrary partition $\lambda=\left(\lambda_{1}, \lambda_{2}, \ldots, \lambda_{l}\right)$ the Schur function $s_{\lambda}(t)$ is defined by

$$
s_{\lambda}(t)=\operatorname{det}\left(s_{\left(\lambda_{i}-i+j\right)}(t)\right)_{1 \leq i, j \leq l} .
$$

Assign the weight $i$ for $t_{i}$. Then $s_{\lambda}(t)$ is homogeneous of the weight $|\lambda|=\sum \lambda_{i}$.

The tau function of a frame $\xi$ of a point $U$ of UGM is defined by

$$
\tau(t ; \xi)=\sum_{\lambda} \xi_{\lambda} s_{\lambda}(t)
$$


where the sum is over all partitions. If $\xi^{\prime}$ is another frame of $U$ then $\tau\left(t ; \xi^{\prime}\right)$ is a constant multiple of $\tau(t ; \xi)$.

The tau function gives a solution of the KP-hierarchy. The KP-hierarchy in the bilinear form is the equation for $\tau(t), t=\left(t_{1}, t_{2}, \ldots\right)^{t}$ given by

$$
\oint \mathrm{e}^{-2 \sum_{j=1}^{\infty} s_{j} z^{j}} \tau\left(t-s-\left[z^{-1}\right]\right) \tau\left(t+s+\left[z^{-1}\right]\right) \frac{\mathrm{d} z}{2 \pi i}=0,
$$

where $s=\left(s_{1}, s_{2}, s_{3}, \ldots\right)^{t},[z]=\left(z, z^{2} / 2, z^{3} / 3, \ldots\right)$ and the integral signifies to take the coefficient of $z^{-1}$ in the formal series expansion of the integrand in $z$.

For a solution $\tau(t)$ of the KP-hierarchy $u(t)=2 \partial_{t_{1}}^{2} \log \tau(t)$ satisfies the KP equation

$$
3 u_{t_{2} t_{2}}+\left(-4 u_{t_{3}}+6 u u_{t_{1}}+u_{t_{1} t_{1} t_{1}}\right)_{t_{1}}=0
$$

Sato's fundamental theorem tells that any formal power series solutions of the KPhierarchy can be constructed from UGM.

Theorem 2.3. 41] For any $U \in \mathrm{UGM}$ and a frame $\xi$ of $U \tau(t ; \xi)$ is a solution of the KP-hierarchy. Conversely for any formal power series solution $\tau(t)$ of the KP-hierarchy there exists a unique point $U$ of UGM and a frame $\xi$ of $U$ such that $\tau(t)=\tau(t ; \xi)$.

The point $U$ of UGM corresponding to a solution $\tau(t)$ in Theorem 2.3 is constructed in the following manner [41, 23, 35].

Let $\Psi^{*}(t ; z)$ be the adjoint wave function [13] corresponding to $\tau(t)$ which is defined by

$$
\Psi^{*}(t ; z)=\frac{\tau(t+[z])}{\tau(t)} \exp \left(-\sum_{i=1}^{\infty} t_{i} z^{-i}\right)
$$

Define $\Psi_{i}^{*}(z)$ by the following expansion

$$
\begin{aligned}
\left.\left(\tau(t) \Psi^{*}(t ; z)\right)\right|_{t=(x, 0,0,0, \ldots)} & =\tau((x, 0,0,0, \ldots)+[z]) \exp \left(-x z^{-1}\right) \\
& =\sum_{i=0}^{\infty} \Psi_{i}^{*}(z) x^{i} .
\end{aligned}
$$

Then

$$
U=\sum_{i=0}^{\infty} \mathbb{C} \Psi_{i}^{*}(z)
$$

\section{Tau functions of hyperelliptic curves}

In this section we describe frames of UGM corresponding to some spaces of functions on hyperelliptic curves. Using them we derive the expression of the degenerate limit of a tau function of a hyperelliptic curve of genus $g$ in terms of tau functions of a hyperelliptic curve of genus $g-1$. 


\subsection{The case of zero point}

Here we construct a frame of UGM from a hyperelliptic curve of genus $g$.

Consider the hyperelliptic curve $C$ of genus $g$ given by

$$
y^{2}=\prod_{j=1}^{2 g+1}\left(x-\alpha_{j}\right)
$$

where $\left\{\alpha_{i}\right\}$ are mutually distinct complex numbers. The space of meromorphic functions on $C$ which are regular on $C \backslash\{\infty\}$ is denoted by $H^{0}(C, \mathcal{O}(* \infty))$. We set

$$
\widetilde{W}=H^{0}(C, \mathcal{O}(* \infty)) .
$$

A basis of $\widetilde{W}$ is given by

$$
x^{i}, \quad i \geq 0, \quad x^{i} y, \quad i \geq 0 .
$$

We take the local coordinate $z$ around $\infty$ such that

$$
x=z^{-2}, \quad y=z^{-(2 g+1)} F(z), \quad F(z)=\left(\prod_{j=1}^{2 g+1}\left(1-\alpha_{j} z^{2}\right)\right)^{1 / 2}
$$

where $F(z)$ is considerer as a power series in $z$ by the Taylor expansion at $z=0$. By expanding functions in $z$ we consider $\widetilde{W}$ as a subspace of $V=\mathbb{C}((z))$. Then (see Corollary 1 of 38 ]

$$
z^{g} \widetilde{W} \in \mathrm{UGM}
$$

Writing the basis (3.3) in terms of $z$ and multiplying them by $z^{g}$ we get a basis of $z^{g} \widetilde{W}$,

$$
z^{g-2 i}, \quad i \geq 0, \quad z^{-g-1-2 i} F(z), \quad i \geq 0 .
$$

If a Laurent series $v(z)$ is of the form

$$
v(z)=\sum_{n \geq N} a_{n} z^{n}, \quad a_{N} \neq 0,
$$

we define the order of $v(z)$ to be $-N$. Arrange elements of (3.6) by their orders and denote them by $\widetilde{f}_{1}, \widetilde{f}_{2}, \ldots$ Explicitly

$$
\begin{aligned}
\left(\widetilde{f}_{1}, \ldots, \widetilde{f}_{g}\right) & =\left(z^{g}, z^{g-2}, z^{g-4}, \ldots, z^{-g+2}\right), \\
\widetilde{f}_{g+2 i+1} & =z^{-g-2 i}, \quad i \geq 0 \\
\widetilde{f}_{g+2 i+2} & =z^{-g-1-2 i} F(z), \quad i \geq 0 .
\end{aligned}
$$

Define the frame $\widetilde{X}$ of a point of UGM by

$$
\widetilde{X}=\left[\ldots, \widetilde{f}_{3}, \widetilde{f}_{2}, \widetilde{f}_{1}\right] .
$$

Notice that $\widetilde{X}$ satisfies (2.1). 


\subsection{Degeneration}

We study the degenerations of the curve $C$ and the frame $\widetilde{X}$.

Let $\alpha$ be a complex number which is different from $\alpha_{j}, 1 \leq j \leq 2 g-1$. We consider the limit of $C$ when $\alpha_{2 g}$ and $\alpha_{2 g+1}$ go to $\alpha$. The curve $C$ tends to

$$
y^{2}=(x-\alpha)^{2} \prod_{j=1}^{2 g-1}\left(x-\alpha_{j}\right) .
$$

The limit of the function $F(z)$ becomes

$$
F(z)=\left(1-\alpha z^{2}\right) F_{0}(z), \quad F_{0}(z)=\left(\prod_{j=1}^{2 g-1}\left(1-\alpha_{j} z^{2}\right)\right)^{1 / 2}
$$

where $F_{0}(z)$ is again considered as a series in $z$. Then the limit of $\tilde{f}_{j}$ exists and it is obtained by replacing $F(z)$ by $\left(1-\alpha z^{2}\right) F_{0}(z)$ in $\tilde{f}_{j}$. Denote the limit of $\tilde{f}_{j}$ by $\widetilde{f}_{j}^{0}$. Define $\widetilde{X}_{0}$ as the limit of the frame $\widetilde{X}$,

$$
\widetilde{X}_{0}=\left[\ldots, \widetilde{f}_{3}^{0}, \widetilde{f}_{2}^{0}, \widetilde{f}_{1}^{0}\right] .
$$

It still is a frame of a point of a UGM, since the highest order terms of $f_{j}$ and $\widetilde{f}_{j}^{0}$ are the same. Let $\widetilde{U}_{0}$ be the corresponding point of UGM.

We multiply $\widetilde{X}_{0}$ by $\left(1-\alpha z^{2}\right)^{-1}$. Then the following set of functions is a basis of $\left(1-\alpha z^{2}\right)^{-1} \widetilde{U}_{0}$

$$
\frac{z^{g-2 i}}{1-\alpha z^{2}}, \quad i \geq 0, \quad z^{-g-1-2 i} F_{0}(z), \quad i \geq 0 .
$$

Lemma 3.1. We have the following equation

$$
\left\langle\frac{z^{g-2 i}}{1-\alpha z^{2}}, \quad i \geq 0\right\rangle=\left\langle\frac{z^{g}}{1-\alpha z^{2}}, z^{g-2-2 i}, \quad i \geq 0\right\rangle,
$$

where, for a set of functions $S,<S>$ denotes the vector space generated by $S$.

Proof. Notice that $z^{g} /\left(1-\alpha z^{2}\right)$ belongs to both the LHS and the RHS of the equation (3.10). Then using the relation

$$
\frac{z^{g-2 i}-\alpha z^{g-2(i-1)}}{1-\alpha z^{2}}=z^{g-2 i}
$$

it is easy to show that the RHS is included in the LHS by induction on $i$. The converse inclusion relation can be proved by using

$$
\frac{z^{g-2(i+1)}}{1-\alpha z^{2}}=z^{g-2(i+1)}+\frac{\alpha z^{g-2 i}}{1-\alpha z^{2}} .
$$


By Lemma 3.1 and (3.9) the set of functions

$$
\frac{z^{g}}{1-\alpha z^{2}}, \quad z^{g-2-2 i}(i \geq 0), \quad z^{-g-1-2 i} F_{0}(z)(i \geq 0) .
$$

becomes a basis of $\left(1-\alpha z^{2}\right)^{-1} \widetilde{U}_{0}$. Arrange the functions in (3.12) by their orders and denote them by $f_{1}, f_{2}, \ldots$,

$$
\begin{aligned}
\left(f_{1}, f_{2}, f_{3}, \ldots, f_{g}\right) & =\left(\frac{z^{g}}{1-\alpha z^{2}}, z^{g-2}, z^{g-4}, \ldots, z^{-g+2}\right) \\
f_{g+2 i+1} & =z^{-g-2 i}, \quad i \geq 0, \\
f_{g+2 i+2} & =z^{-g-2 i-1} F_{0}(z), \quad i \geq 0 .
\end{aligned}
$$

Define the frame $X$ of UGM by

$$
X=\left(X_{i, j}\right)_{i \in \mathbb{Z}, j \in \mathbb{N}}=\left[\ldots, f_{3}, f_{2}, f_{1}\right] .
$$

In the change from (3.9) to (3.12) only the relation (3.11) is used. It does not change the Plücker coordinates. Therefore the tau functions of $X$ and $\left(1-\alpha z^{2}\right)^{-1} \widetilde{X}_{0}$ are the same,

$$
\tau(t ; X)=\tau\left(t ;\left(1-\alpha z^{2}\right)^{-1} \widetilde{X}_{0}\right) .
$$

\subsection{The case of one point}

In this section we study the frames of UGM corresponding to the space of functions on a hyperelliptic curve of genus $g-1$ which have at most a simple pole at some fixed point and a pole at $\infty$ of arbitrary order. We use them to describe the frame $X$.

Consider the hyperelliptic curve $C^{\prime}$ of genus $g-1$ given by

$$
y^{2}=\prod_{j=1}^{2 g-1}\left(x-\alpha_{j}\right)
$$

which is extracted from the singular curve (3.8).

Let $z$ be the local coordinate of $C^{\prime}$ around $\infty$ such that

$$
x=z^{-2}, \quad y=z^{-(2 g+1)} F_{0}(z)
$$

and $\alpha$ be as in the previous section.

Fix a point $\left(\alpha, y_{0}\right)$ of $C^{\prime}$ and set

$$
p_{ \pm}=\left(\alpha, \pm y_{0}\right)
$$

Since $\alpha$ is different from $\alpha_{j}, 1 \leq j \leq 2 g-1, y_{0} \neq 0$ and $p_{+} \neq p_{-}$.

For a point $P$ of $C^{\prime}$ denote by $H^{0}\left(C^{\prime}, \mathcal{O}(P+* \infty)\right)$ the space of meromorphic functions on $C^{\prime}$ which are regular on $C^{\prime} \backslash\{P, \infty\}$ and have a pole at $P$ of order at most one. Define the vector spaces $W_{ \pm}$by

$$
W_{ \pm}=H^{0}\left(C^{\prime}, \mathcal{O}\left(p_{ \pm}+* \infty\right)\right)
$$


We consider $W_{ \pm}$as a subspace of $V=\mathbb{C}((z))$ by expanding elements in $W \pm$ in $z$. Then (see Corollary 1 of [38])

$$
z^{g-2} W_{ \pm} \in \mathrm{UGM}
$$

The following elements give a basis $z^{g-2} W_{ \pm}$,

$$
z^{g-2-2 i}, \quad i \geq 0, \quad z^{-g-1-2 i} F_{0}(z), \quad i \geq 0, \quad z^{-g+1} \frac{F_{0}(z) \pm y_{0} z^{2 g-1}}{1-\alpha z^{2}} .
$$

Arrange the functions in (3.19) by their orders and name them as $h_{1}^{ \pm}, h_{2}^{ \pm}, \ldots$,

$$
\begin{aligned}
\left(h_{1}^{ \pm}, h_{2}^{ \pm}, \ldots, h_{g}^{ \pm}\right) & =\left(z^{g-2}, z^{g-4}, \ldots, z^{-g+2}, z^{-g+1} \frac{F_{0}(z) \pm y_{0} z^{2 g-1}}{1-\alpha z^{2}}\right) \\
h_{g+2 i+1}^{ \pm} & =z^{-g-2 i}, \quad i \geq 0, \\
h_{g+2 i+2}^{ \pm} & =z^{-g-1-2 i} F_{0}(z), \quad i \geq 0 .
\end{aligned}
$$

We define the frames $Y^{ \pm}$of points of UGM by

$$
Y^{ \pm}=\left[\ldots, h_{3}^{ \pm}, h_{2}^{ \pm}, h_{1}^{ \pm}\right]
$$

\subsection{Relation of tau functions}

We express the tau function corresponding to the frame $X$ in terms of the tau functions corresponding to the frames $Y^{ \pm}$.

Since $h_{i}^{+}$and $h_{i}^{-}$coincide except $i=g$, we set, for $i \neq g$,

$$
h_{i}=h_{i}^{+}=h_{i}^{-} \text {. }
$$

Then $h_{i}$ is related to $f_{i}$ by

$$
h_{i}= \begin{cases}f_{i+1} & 1 \leq i \leq g-1 \\ f_{i} & i \geq g+1\end{cases}
$$

The following important decomposition holds,

$$
f_{1}=\left(2 y_{0}\right)^{-1}\left(h_{g}^{+}-h_{g}^{-}\right) .
$$

Substituting (3.22) into the frame $X$, for any partition $\lambda$, the Plücker coordinate $X_{\lambda}$ can be written in terms of the Plücker coordinates $Y_{\lambda}^{ \pm}$as

$$
X_{\lambda}=(-1)^{g-1}\left(2 y_{0}\right)^{-1}\left(Y_{\lambda}^{+}-Y_{\lambda}^{-}\right) .
$$

Thus we have

Theorem 3.2. Let $C$ be the hyperelliptic curve of genus $g$ defined by (3.1) and $\tilde{X}$ the frame defined by (3.7) corresponding to $C$. Consider the degeneration of $C$ to the curve defined by (3.8) with one double point. Denote by $X$ the frame defined by (3.13) which is obtained from the limit of $\widetilde{X}$ and by $Y^{ \pm}$the frames defined by (3.20) corresponding to the non-singular curve of genus $g-1$ defined by (3.15).

Then

$$
\tau(t ; X)=(-1)^{g-1}\left(2 y_{0}\right)^{-1}\left(\tau\left(t ; Y^{+}\right)-\tau\left(t ; Y^{-}\right)\right) .
$$




\section{Tau function in terms of sigma function}

In the previous section frames $\tilde{X}, \widetilde{X}_{0}, Y^{ \pm}$of points of UGM are introduced. The tau functions corresponding to those frames are defined as series as in (2.3). There are analytic expressions of these tau functions in terms of multi-variable sigma functions. In this section we give them.

\subsection{Hyperelliptic sigma function}

Sigma functions are defined for an arbitrary Riemann surface [26, 36]. To recall the general case is too much for our purpose. On the other hand, in the case of hyperelliptic curves, everything is explicit and is easy to understand. So we restrict ourselves to the hyperelliptic curve $C$ and recall the definition and properties of the sigma function of $C$. To this end we need period matrices of certain first and second kind differentials [7, 34].

Write the right hand side of (3.1) as

$$
\prod_{j=1}^{2 g+1}\left(x-\alpha_{j}\right)=\sum_{i=0}^{2 g+1} \mu_{4 g+2-2 i} x^{i}, \quad \mu_{0}=1 .
$$

We assign the weight $k$ to $\mu_{k}$, and weight 2 to $x$. Then this polynomial is homogeneous of the weight $4 g+2$.

Let $\left\{\mathrm{d} u_{2 i-1}^{(g)} \mid 1 \leq i \leq g\right\}$ be a basis of holomorphic one forms given by

$$
\mathrm{d} u_{2 i-1}^{(g)}=\frac{x^{g-i} \mathrm{~d} x}{-2 y}
$$

and $\widehat{\Omega}^{(g)}\left(p_{1}, p_{2}\right)$ the bidifferential on $C \times C$ defined by

$$
\begin{aligned}
\widehat{\Omega}^{(g)}\left(p_{1}, p_{2}\right) & =\frac{2 y_{1} y_{2}+\sum_{i=0}^{g} x_{1}^{i} x_{2}^{i}\left(2 \mu_{4 g+2-4 i}+\mu_{4 g-4 i}\left(x_{1}+x_{2}\right)\right)}{4\left(x_{1}-x_{2}\right)^{2} y_{1} y_{2}} \mathrm{~d} x_{1} \mathrm{~d} x_{2} \\
& =\mathrm{d}_{p_{2}}\left(\frac{y_{1}+y_{2}}{2 y_{1}\left(x_{1}-x_{2}\right)}\right)+\sum_{i=1}^{g} \mathrm{~d} u_{2 i-1}^{(g)}\left(p_{1}\right) \mathrm{d} r_{2 i-1}^{(g)}\left(p_{2}\right)
\end{aligned}
$$

where $p_{i}=\left(x_{i}, y_{i}\right) \in C$ and

$$
\mathrm{d} r_{2 i-1}^{(g)}=\frac{\mathrm{d} x}{-2 y} \sum_{k=1}^{2 i-1} k \mu_{4 i-2 k-2} x^{k+g-i}
$$

The differential $\mathrm{d} r_{2 i-1}^{(g)}$ is a second kind differential with a pole only at $\infty$ and $\left\{\mathrm{d} u_{2 i-1}^{(g)}, \mathrm{d} r_{2 j-1}^{(g)}\right\}$ forms a symplectic basis of $H^{1}(C, \mathbb{C})$ with respect to the intersection form [35].

Take a canonical homology basis $\left\{\mathfrak{a}_{i}, \mathfrak{b}_{i} \mid 1 \leq i \leq g\right\}$ of the first homology group of $C$ and define the $g \times g$ period matrices by

$$
2 \omega^{(g)}=\left(\int_{\mathfrak{a}_{j}} \mathrm{~d} u_{2 i-1}^{(g)}\right), \quad 2 \omega^{\prime(g)}=\left(\int_{\mathfrak{b}_{j}} \mathrm{~d} u_{2 i-1}^{(g)}\right),
$$




$$
\begin{aligned}
& -2 \eta^{(g)}=\left(\int_{\mathfrak{a}_{j}} \mathrm{~d} r_{2 i-1}^{(g)}\right), \quad-2 \eta^{\prime(g)}=\left(\int_{\mathfrak{b}_{j}} \mathrm{~d} r_{2 i-1}^{(g)}\right), \\
& T^{(g)}=\left(\omega^{(g)}\right)^{-1} \omega^{\prime(g)} .
\end{aligned}
$$

With the normalized period matrix $T^{(g)}$ the Riemann theta function with characteristics is defined by

$$
\theta\left[\begin{array}{c}
\varepsilon \\
\varepsilon^{\prime}
\end{array}\right]\left(v \mid T^{(g)}\right)=\sum_{n \in \mathbb{Z}^{g}} \exp \left(\pi i\left(n+\varepsilon^{\prime}\right)^{t} T^{(g)}\left(n+\varepsilon^{\prime}\right)+2 \pi i\left(n+\varepsilon^{\prime}\right)^{t}(v+\varepsilon)\right),
$$

where $\varepsilon, \varepsilon^{\prime} \in \mathbb{R}^{g}$. To characteristics $\varepsilon, \varepsilon^{\prime} \in \mathbb{R}^{g}$ a vector $\vec{v} \in \mathbb{C}^{g}$ is associated by

$$
v=I^{(g)} \varepsilon+T^{(g)} \varepsilon^{\prime}
$$

where $I^{(g)}$ is the unit matrix of degree $g$. This gives a bijection between the set of characteristics and the vectors in $\mathbb{C}^{g}$. For a vector $v \in \mathbb{C}^{g}$ we denote $[v]$ the characteristics $\left[\begin{array}{c}\varepsilon \\ \varepsilon^{\prime}\end{array}\right]$.

Choose $\infty$ as a base point and denote by $K$ the Riemann's constant for this choice. Let $[-K]=\left[\begin{array}{l}\kappa \\ \kappa^{\prime}\end{array}\right]$. It is known that $[-K]$ is a half period, that is, all components of $\kappa, \kappa^{\prime}$ are half integers (see [7, 33] for example).

In the definition of the sigma function we need some normalization constant which is described from a certain derivative of $\theta[-K]\left(\left(2 \omega^{(g)}\right)^{-1} u \mid T^{(g)}\right), u=\left(u_{1}, u_{3}, \ldots, u_{2 g-1}\right)^{t}$. We recall necessary results concerning to it.

Set

$$
\begin{aligned}
& m_{k}^{(g)}=\left[\frac{g+1-k}{2}\right], \quad 0 \leq k \leq g, \\
& \left(w_{1}, \ldots, w_{g}\right)=(1,3,5, \ldots, 2 g-1), \quad\left(w_{1}^{*}, \ldots, w_{g}^{*}\right)=(0,2,4, \ldots, 2 g-2) .
\end{aligned}
$$

For $0 \leq k \leq g-1$ define

$$
\begin{aligned}
& A_{k}^{(g)}=\left(a_{k, 1}^{(g)}, \ldots, a_{k, m_{k}^{(g)}}^{(g)}\right)=(2 g-2 k-1,2 g-2 k-5,2 g-2 k-9, \ldots), \\
& \mathrm{s}_{k}^{(g)}=\operatorname{sgn}\left(\begin{array}{cccccc}
w_{1}^{*} & \ldots & w_{m_{k}^{*}(g)}^{*} & w_{g-k-m_{k}^{(g)}} & \ldots & w_{1} \\
g-k-1 & & \ldots & \ldots & 1 & 0
\end{array}\right) .
\end{aligned}
$$

We set $A_{g}^{(g)}=\emptyset, s_{g}^{(g)}=1$. The following relation is valid,

$$
A_{k}^{(g)}=A_{0}^{(g-k)} .
$$

Example 4.1. $A_{2}^{(3)}=A_{1}^{(2)}=A_{0}^{(1)}=(1)$,

$A_{2}^{(4)}=A_{1}^{(3)}=A_{0}^{(2)}=(3)$,

$A_{2}^{(5)}=A_{1}^{(4)}=A_{0}^{(3)}=(5,1)$,

$A_{2}^{(6)}=A_{1}^{(5)}=A_{0}^{(4)}=(7,3)$. 
Remark 4.2. The number $A_{k}^{(g)}$ was introduced in [40] by different notation for hyperelliptic curves. Its generalizations are given in [39] for $(n, s)$ curves and in [36] for arbitrary Riemann surfaces.

In general, for $A=\left(a_{1}, \ldots, a_{r}\right) \in\{1,3, \ldots, 2 g-1\}^{r}$ and a function $G(u), u=\left(u_{1}, u_{3}\right.$, $\left.\ldots, u_{2 g-1}\right)^{t}$, let

$$
\begin{aligned}
\partial_{A} & =\partial_{u_{a_{1}}} \cdots \partial_{u_{a_{r}}}, \quad G_{A}(u)=\partial_{A} G(u), \quad \partial_{u_{a}}=\frac{\partial}{\partial u_{a}}, \\
|A| & =a_{1}+\cdots+a_{r} .
\end{aligned}
$$

For $A=\emptyset$ we set $G_{A}(u)=G(u)$.

Some of the properties of $A_{k}^{(g)}$ are

Lemma 4.3. [40] (i) $\left|A_{k}^{(g)}\right|=\frac{1}{2}(g+1-k)(g-k)$.

(ii) Let $\nu^{(g)}=(g, g-1, \ldots, 1)$. Then

$$
\partial_{A_{0}^{(g)}} s_{\nu(g)}(t)=s_{0}^{(g)}
$$

(iii) Suppose that $A \in\{1,3, \ldots, 2 g-1\}^{r}, r \geq 1$ and $\nu$ be a partition. If $|A|<|\nu|$ then $\left(\partial_{A} s_{\nu}\right)(0)=0$.

Notice that the property (iii) follows from the fact that the Schur function $s_{\nu}(t)$ is a weight homogeneous polynomial with the weight $|\nu|$. We include it in the lemma for the sake of later use.

The following theorem is proved in [40].

Theorem 4.4. [40] $\theta[-K]_{A_{0}^{(g)}}\left(0 \mid T^{(g)}\right) \neq 0$.

Remark 4.5. Lemma 4.3 and Theorem 4.4 have been generalized to the case of $(n, s)$ curves in [39] and to the case of arbitrary Riemann surfaces in [36].

Definition 4.6. The fundamental sigma function of the hyperelliptic curve $C$ is defined for the data $\left(C, \infty,\left\{\mathrm{d} u_{2 i-1}^{(g)}\right\}, \widehat{\Omega}^{(g)}\right)$ by

$$
\sigma^{(g)}(u)=\exp \left(\frac{1}{2} u^{t} \eta^{(g)}\left(\omega^{(g)}\right)^{-1} u\right) \frac{\theta[-K]\left(\left(2 \omega^{(g)}\right)^{-1} u \mid T^{(g)}\right)}{\mathrm{s}_{0}^{(g)} \theta[-K]_{A_{0}^{(g)}}\left(0 \mid T^{(g)}\right)},
$$

where $u=\left(u_{1}, u_{3}, \ldots, u_{2 g-1}\right)^{t}$.

In the remaining part of the paper we call the fundamental sigma function simply the sigma function for the sake of simplicity.

Similarly to Riemann's theta function the sigma function is a quasi-periodic function.

Lemma 4.7. 24, 25, 7] For $m_{1}, m_{2} \in \mathbb{Z}^{g}$ the sigma function satisfies the following relation,

$$
\begin{aligned}
& \sigma^{(g)}\left(u+\omega^{(g)} m_{1}+\omega^{\prime(g)} m_{2}\right) \\
& =(-1)^{m_{1}^{t} m_{2}+2\left(\kappa^{t} m_{1}-\kappa^{\prime t} m_{2}\right)} \\
& \times \exp \left(2\left(\eta^{(g)} m_{1}+\eta^{\prime(g)} m_{2}\right)^{t}\left(u+\omega^{(g)} m_{1}+\omega^{\prime(g)} m_{2}\right)\right) \sigma^{(g)}(u) .
\end{aligned}
$$


Assign the weight $i$ to $u_{i}$. Then the sigma function has several nice properties compared with Riemann's theta function.

Theorem 4.8. 24, 25, 8, 34]

(i) The function $\sigma^{(g)}(u)$ does not depend on the choice of a canonical homology basis.

(ii) Taylor coefficients of $\sigma^{(g)}(u)$ are weighted homogeneous polynomials of $\left\{\mu_{i}\right\}$ with the coefficients in $\mathbb{Q}$.

(iii) The following expansion is valid,

$$
\sigma^{(g)}(u)=s_{\nu^{(g)}}(u)+(\text { terms with weights }>|\nu|),
$$

where $\nu^{(g)}=(g, g-1, \ldots, 1)$.

Remark 4.9. (i) Due to (i) of Theorem 4.8 the choice of a canonical homology basis is not included in the data to define the sigma function in Definition 4.6. The property (i) signifies that the sigma function is modular invariant, since the change of a canonical homology basis is described by the action of the symplectic group $\operatorname{Sp}(2 g, \mathbb{Z})$.

(ii) The sigma function for a hyperelliptic curve had been introduced by Klein [24, 25]. It coincides with the Weierstrass sigma function if $g=1$ and the equation of $C$ is given in the Weierstarss canonical form. Theorem 4.8 is essentially due to Klein. But the relation with Schur functions was discovered in [8] and proved in [34. The definition of sigma functions are generalized to $(n, s)$ curves in [8], to telescopic curves in [2] and to arbitrary Riemann surfaces in [26]. The properties of sigma functions corresponding to Theorem 4.8 had been established in [34, 35] for $(n, s)$ curves, in [2] for telescopic curves, and the properties corresponding to (i), (iii) of Theorem 4.8 were proved in [36] for any Riemann surface.

\subsection{Tau function corresponding to the frame $\widetilde{X}$}

In this section we recall the representation of the tau function corresponding to the point $z^{g} \widetilde{W}$ of UGM in terms of the fundamental sigma function [35].

Define $b_{i j}^{(g)}, \widehat{q}_{i j}^{(g)}$ and $c_{i}^{(g)}$ by the expansions at $\infty$,

$$
\begin{aligned}
\mathrm{d} u_{2 i-1}^{(g)} & =\left(\sum_{j=1}^{\infty} b_{i j}^{(g)} z^{j-1}\right) \mathrm{d} z \\
\widehat{\Omega}^{(g)}\left(p_{1}, p_{2}\right) & =\left(\frac{1}{\left(z_{1}-z_{2}\right)^{2}}+\sum_{i, j=1}^{\infty} \widehat{q}_{i j}^{(g)} z_{1}^{i-1} z_{2}^{j-1}\right) \mathrm{d} z_{1} \mathrm{~d} z_{2}, \\
\log \left(z^{-g+1} \sqrt{\frac{\mathrm{d} u_{2 g-1}^{(g)}}{\mathrm{d} z}}\right) & =\sum_{i=1}^{\infty} c_{i}^{(g)} \frac{z^{i}}{i} .
\end{aligned}
$$

Using

$$
\mathrm{d} u_{2 i-1}^{(g)}=\frac{z^{2 i-2} \mathrm{~d} z}{F(z)}
$$


$c_{i}^{(g)}$ can be expressed explicitly by $\left\{\alpha_{j}\right\}$ as follows. We have

$$
\begin{aligned}
\log \left(z^{-g+1} \sqrt{\frac{\mathrm{d} u_{2 g-1}^{(g)}}{\mathrm{d} z}}\right) & =-\frac{1}{2} \log F(z) \\
& =-\frac{1}{4} \sum_{j=1}^{2 g+1} \log \left(1-\alpha_{j} z^{2}\right) \\
& =\sum_{i=1}^{\infty}\left(\frac{1}{2} \sum_{j=1}^{2 g+1} \alpha_{j}^{i}\right) \frac{z^{2 i}}{2 i}
\end{aligned}
$$

Thus

$$
c_{i}^{(g)}= \begin{cases}0 & \text { if } i \text { is odd } \\ \frac{1}{2} \sum_{j=1}^{2 g+1} \alpha_{j}^{i / 2} & \text { if } i \text { is even }\end{cases}
$$

Define

$$
B^{(g)}=\left(b_{i j}^{(g)}\right)_{1 \leq i \leq g, j \geq 1}, \quad \widehat{q}^{(g)}(t)=\sum_{i, j=1}^{\infty} \widehat{q}_{i j}^{(g)} t_{i} t_{j} .
$$

Then

Theorem 4.10. 35. The tau function corresponding to the frame $\tilde{X}$ defined by (3.7) has the following expression

$$
\tau(t ; \widetilde{X})=\exp \left(-\sum_{i=1}^{\infty} c_{2 i}^{(g)} t_{2 i}+\frac{1}{2} \widehat{q}^{(g)}(t)\right) \sigma^{(g)}\left(B^{(g)} t\right) .
$$

Remark 4.11. In [35] this theorem is proved for any $(n, s)$ curve.

\subsection{Tau function corresponding to the frames $Y^{ \pm}$}

In this section we derive the sigma function expression of the tau function corresponding to the frames $Y^{ \pm}$of UGM. In $§ 3.3 p_{ \pm}=\left(\alpha, \pm y_{0}\right)$ was introduced as points on the curve $C^{\prime}$ of genus $g-1$. In this section $p_{ \pm}$denote points of $C$ except in Theorem 4.18, where $C^{\prime}$ is considered, for the sake of notational simplicity

For $p \in C$ set

$$
u_{j}^{(g)}(p)=\int_{\infty}^{p} \mathrm{~d} u_{j}^{(g)}, \quad u^{(g)}(p)=\left(u_{1}^{(g)}(p), u_{3}^{(g)}(p), \ldots, u_{2 g-1}^{(g)}(p)\right)^{t} .
$$

We omit the superscript $(g)$ of $u_{j}^{(g)}(p), u^{(g)}(p)$ and denote them by $u_{j}(p), u(p)$ respectively if there is no fear of confusion. 
Proposition 4.12. 40] Let $1 \leq k \leq g$.

(i) Let $A \subset\{1,3, \ldots, 2 g-1\}^{r}, r \geq 1$. If $|A|<\left|A_{k}^{(g)}\right|$ then

$$
\sigma_{A}^{(g)}\left(u\left(p_{1}\right)+\cdots+u\left(p_{k}\right)\right)=0
$$

for all $p_{1}, \ldots, p_{k} \in C$.

(ii) As a function of $p_{1}, \ldots, p_{k} \in C,\left(\sigma^{(g)}\right)_{A_{k}^{(g)}}\left(u\left(p_{1}\right)+\cdots+u\left(p_{k}\right)\right)$ is not identically zero.

(iii) The following expansion in $z_{k}=z\left(p_{k}\right)$ holds,

$$
\begin{aligned}
\sigma_{A_{k}^{(g)}}^{(g)}\left(u\left(p_{1}\right)\right. & \left.+\cdots+u\left(p_{k}\right)\right) \\
& =\mathrm{s}_{k, k-1}^{(g)} \sigma_{A_{k-1}^{(g)}}^{(g)}\left(u\left(p_{1}\right)+\cdots+u\left(p_{k-1}\right)\right) z_{k}^{g+1-k}+O\left(z_{k}^{g+2-k}\right),
\end{aligned}
$$

where $\mathrm{s}_{k, k-1}^{(g)}=\mathrm{s}_{k}^{(g)} \mathrm{s}_{k-1}^{(g)}$ and, for $k=1,\left(\sigma^{(g)}\right)_{A_{k-1}^{(g)}}\left(u\left(p_{1}\right)+\cdots+u\left(p_{k-1}\right)\right)$ should be understood as 1 .

Remark 4.13. This proposition is generalized to the case of $(n, s)$ curves in [39]. In [36] the equivalent assertions to this proposition have been proved for the Riemann theta function of any Riemann surface.

Since $p_{+}$is the image of $p_{-}$by the hyperelliptic involution $(x, y) \rightarrow(x,-y)$,

$$
u\left(p_{-}\right)=-u\left(p_{+}\right) .
$$

Therefore, we have, by the case $k=2$ of Proposition 4.12 (iii),

$$
\begin{aligned}
\sigma_{A_{2}^{(g)}}^{(g)}\left(u(p)-u\left(p_{ \pm}\right)\right) & =\sigma_{A_{2}^{(g)}}^{(g)}\left(u(p)+u\left(p_{\mp}\right)\right) \\
& =\mathrm{s}_{2,1}^{(g)} \sigma_{A_{1}^{(g)}}^{(g)}\left(u\left(p_{\mp}\right)\right) z^{g-1}+O\left(z^{g}\right) \\
& =\mathrm{s}_{2,1}^{(g)} \sigma_{A_{1}^{(g)}}^{(g)}\left(-u\left(p_{ \pm}\right)\right) z^{g-1}+O\left(z^{g}\right),
\end{aligned}
$$

where $z=z(p)$.

Proposition 4.14. [40] $\sigma_{A_{1}^{(g)}}^{(g)}\left(-u\left(p_{ \pm}\right)\right) \neq 0$.

Proof. This proposition had been proved in [40]. Since we use s similar argument to that in the proof of this proposition later, we give a proof here.

Set

$$
f(p)=\sigma_{A_{1}^{(g)}}^{(g)}(u(p)) .
$$

By the case $k=1$ of Proposition 4.12 (ii), (iii) it does not vanish identically as a function of $p$ and has the expansion of the form

$$
f(p)=s_{1,0}^{(g)} z^{g}+O\left(z^{g+1}\right) .
$$

Therefore $f(p)$ has a zero of order $g$ at $\infty$. Then the proposition follows from (ii) of the following proposition, since $p_{ \pm} \neq \infty$. 
Proposition 4.15. (i) For a cycle $\gamma=\sum_{i=1}^{g}\left(m_{1, i} \mathfrak{a}_{i}+m_{2, i} \mathfrak{b}_{i}\right), m_{1, i}, m_{2, i} \in \mathbb{Z}$, let $f(\gamma p)$ denote the analytic continuation of $f(p)$ along $\gamma$. Then

$$
\begin{aligned}
& f(\gamma p)=(-1)^{m_{1}^{t} m_{2}+2\left(\kappa^{t} m_{1}-\kappa^{\prime t} m_{2}\right)} \\
& \times \exp \left(2\left(\eta^{(g)} m_{1}+\eta^{\prime(g)} m_{2}\right)^{t}\left(u(p)+\omega^{(g)} m_{1}+\omega^{\prime(g)} m_{2}\right)\right) f(p),
\end{aligned}
$$

where $m_{i}=\left(m_{i, 1}, \ldots, m_{i, g}\right)^{t}$.

(ii) The function $f(p)$ has precisely $g$ zeros on $C$.

Remark 4.16. Since $f(p)$ is a composition of the Abel-Jacobi map and the analytic function $\sigma_{A_{1}^{(g)}}^{(g)}(u)$ on $\mathbb{C}^{g}$, it is a multi-valued analytic function on $C$. The property (i) of Proposition 4.15 means that $f(p)$ is in fact a quasi-periodic function. Then whether $f(p)$ becomes zero or not does not depend on the choice of a branch of $f(p)$. Therefore the assertion of Proposition 4.15, (ii) makes sense.

Proof of Proposition 4.15

(i) Apply the derivative $\partial_{A_{1}^{(g)}}$ to both hand sides of (4.8) and set $u=u(p)$. Then the property (i) of Proposition 4.12 implies the formula (4.15).

(ii) For the proof let us recall the following well-known property of Riemann's theta function (see [19], 32] for example).

Theorem 4.17. Let $C$ be a compact Riemann surface of genus $g, p_{0}$ a point of $C$, $\left\{\mathfrak{a}_{i}, \mathfrak{b}_{i} \mid 1 \leq i \leq g\right\}$ a canonical homology basis, $d v^{(g)}=\left(d v_{1}^{(g)}, \ldots, d v_{g}^{(g)}\right)^{t}$ the normalized basis of holomorphic one forms, $\varepsilon, \varepsilon^{\prime} \in \mathbb{R}^{g}, v \in \mathbb{C}^{g}$ and $\widetilde{f}(p)=\theta\left[\begin{array}{l}\varepsilon \\ \varepsilon^{\prime}\end{array}\right]\left(\int_{p_{0}}^{p} d v^{(g)}+v\right)$. If $\widetilde{f}(p)$ does not vanish identically, then it has precisely $g$ zeros on $C$.

In the present case of the hyperelliptic curve $C$ we use the same notation $\left\{d v_{j}^{(g)}\right\}$ for the basis of the normailzed holomorphic one forms and set, for $p \in C$,

$$
v_{j}^{(g)}(p)=\int_{\infty}^{p} d v_{j}^{(g)}, \quad v^{(g)}(p)=\left(v_{1}^{(g)}(p), v_{2}(p), \ldots, v_{g}^{(g)}(p)\right)^{t} .
$$

Again the superscript $(g)$ is omitted if there is no fear of confusion.

The sigma function is defined by (4.6) using the Riemann theta function. Conversely Riemann's theta function is expressed by the sigma function as

$$
\theta[-K]\left(v \mid T^{(g)}\right)=C \exp \left(-\frac{1}{2}\left(2 \omega^{(g)} v\right)^{t} \eta^{(g)}\left(\omega^{(g)}\right)^{-1}\left(2 \omega^{(g)} v\right)\right) \sigma^{(g)}\left(2 \omega^{(g)} v\right),
$$

for some constant $C$. With this in mind we define the function $F(p)$ by

$$
F(p)=\exp \left(-\frac{1}{2}\left(2 \omega^{(g)} v(p)\right)^{t} \eta^{(g)}\left(\omega^{(g)}\right)^{-1}\left(2 \omega^{(g)} v(p)\right)\right) \sigma_{A_{1}^{(g)}}^{(g)}\left(2 \omega^{(g)} v(p)\right) .
$$

Then $F(p)$ satisfies

$$
F\left(\mathfrak{a}_{j} p\right)=\exp \left(2 \pi i \kappa_{j}\right) F(p)
$$




$$
F\left(\mathfrak{b}_{j} p\right)=\exp \left(-2 \pi i \kappa_{j}^{\prime}-\pi i T_{j, j}^{(g)}-2 \pi i v_{j}(p)\right) F(p),
$$

where $\kappa_{j}$ is the $j$-th component of $\kappa$. Then exactly the same proof can be applied to $F(p)$ as to $\tilde{f}(p)$ in Theorem 4.17. Therefore $F(p)$ has precisely $g$ zeros on $C$. Since

$$
2 \omega^{(g)} v(p)=u(p)
$$

it follows that $\sigma_{A_{1}^{(g)}}^{(g)}(u(p))$ has $g$ zeros on $C$.

By (4.14) and Proposition 4.14 the following definition of the numbers $d_{j}^{(g, \pm)}, j \geq 1$, makes sense,

$$
\log \left(z^{g-1} \frac{\mathrm{s}_{2,1}^{(g)} \sigma_{A_{1}^{(g)}}^{(g)}\left(-u\left(p_{ \pm}\right)\right)}{\sigma_{A_{2}^{(g)}}^{(g)}\left(u(p)-u\left(p_{ \pm}\right)\right)}\right)-\frac{1}{2} \widehat{q}^{(g)}([z])=\sum_{j=1}^{\infty} d_{j}^{(g, \pm)} \frac{z^{j}}{j}
$$

where, for $g=1$, we set $A_{2}^{(1)}=\emptyset, \mathrm{s}_{2,1}^{(1)}=1$.

Then

Theorem 4.18. The tau function corresponding to the frames $Y^{\epsilon}, \epsilon= \pm$, defined by (3.20) has the following expression

$$
\begin{aligned}
\tau\left(t ; Y^{\epsilon}\right) & =C_{\epsilon} \exp \left(\sum_{j=1}^{\infty} d_{j}^{(g-1, \epsilon)} t_{j}+\frac{1}{2} \widehat{q}^{(g-1)}(t)\right) \sigma^{(g-1)}\left(B^{(g-1)} t-u\left(p_{\epsilon}\right)\right), \\
C_{\epsilon} & =\left(\mathrm{s}_{0}^{(g-2)} \sigma_{A_{0}^{(g-2)}}^{(g-1)}\left(-u\left(p_{\epsilon}\right)\right)\right)^{-1},
\end{aligned}
$$

where $d_{j}^{(g-1, \epsilon)}$ is defined by (4.16) $, B^{(g-1)}, \widehat{q}^{(g-1)}$ are given by (4.10) and $A_{0}^{(g-2)}, s_{0}^{(g-2)}$, $p_{ \pm}$are given by (4.5), (4.6), (3.16) respectively.

Proof. Let us denote the right hand side of (4.17) by $\tau_{\epsilon}(t)$. Notice first that $\tau_{\epsilon}(t)$ is a solution of the KP-hierarchy (2.4). In fact it is proved in [35, 14] that $\widetilde{\tau}(t)$ defined by

$$
\widetilde{\tau}(t)=\exp \left(\frac{1}{2} \widehat{q}^{(g-1)}(t)\right) \sigma^{(g-1)}\left(B^{(g-1)} t+u\right)
$$

is a solution of the KP-hierarchy for any $u \in \mathbb{C}^{g}$. In general if $\tau_{1}(t)$ is a solution of (2.4) then $\tau_{2}(t):=\mathrm{e}^{c_{0}+\sum_{i=1}^{\infty} c_{i} t_{i}} \tau_{1}(t)$ is a solution of (2.4) for arbitrary constants $c_{i}, i \geq 0$. The solution $\tau_{2}(t)$ is called the gauge transformation of $\tau_{1}(t)$. Since $\tau_{\epsilon}(t)$ is a gauge transformation of $\widetilde{\tau}(t)$ with $u=-u^{(g-1)}\left(p_{\epsilon}\right)$, it is a solution of the KP-hierarchy.

In order to determine the point of UGM corresponding to $\tau_{\epsilon}(t)$ according to (2.8) we compute the adjoint wave function (2.6) of $\tau_{\epsilon}(t)$,

$$
\Psi_{\epsilon}^{*}(t ; z)=\frac{\tau_{\epsilon}(t+[z])}{\tau_{\epsilon}(t)} \exp \left(-\sum_{i=1}^{\infty} t_{i} z^{-i}\right) .
$$


Let $\mathrm{d} \widetilde{r}_{i}$ be the normalized differential of the second kind with a pole only at $\infty$ such that

$$
\begin{aligned}
& \mathrm{d} \widetilde{r}_{i}=\mathrm{d}\left(\frac{1}{z^{i}}+O(1)\right) \quad \text { near } \infty \\
& \int_{\mathfrak{a}_{j}} \mathrm{~d} \widetilde{r}_{i}=0 \text { for any } j .
\end{aligned}
$$

Set

$$
\mathrm{d} \widehat{r}_{i}=\mathrm{d} \widetilde{r}_{i}+\sum_{k, l=1}^{g} b_{k, i}\left(\eta^{(g)}\left(\omega^{(g)}\right)^{-1}\right)_{k, l} \mathrm{~d} u_{2 l-1}^{(g)} .
$$

It is proved in Lemma 8 of 35 , that

$$
\mathrm{d} \widehat{r}_{i}=\mathrm{d}\left(\frac{1}{z^{i}}-\sum_{j=1}^{\infty} \widehat{q}_{i, j} \frac{z^{j}}{j}\right) .
$$

By the definition of $B^{(g-1)}$ we have

$$
B^{(g-1)}[z]=u^{(g-1)}(p),
$$

for $z=z(p)$. Using (4.16), (4.20), (4.21) we get

$$
\begin{aligned}
& \Psi_{\epsilon}^{*}(t ; z) \\
& =z^{g-2} \frac{\mathrm{s}_{2,1}^{(g-1)} \sigma_{A_{1}^{(g-1)}}^{(g-1)}\left(-u\left(p_{\epsilon}\right)\right)}{\sigma_{A_{2}^{(g-1)}}^{(g-1)}\left(u(p)-u\left(p_{\epsilon}\right)\right)} \frac{\sigma^{(g-1)}\left(B^{(g-1)} t+u(p)-u\left(p_{\epsilon}\right)\right)}{\sigma^{(g-1)}\left(B^{(g-1)} t-u\left(p_{\epsilon}\right)\right)} \\
& \quad \times \exp \left(-\sum_{i=1}^{\infty} t_{i} \int^{p} \mathrm{~d} \widehat{r}_{i}\right),
\end{aligned}
$$

where $\int^{p} \mathrm{~d} \widehat{r}_{i}$ is the indefinite integral without a constant term in the expansion in $z$. We denote $z^{-(g-2)} \Psi_{\epsilon}^{*}(t ; z)$ by $\Psi_{\epsilon}^{*}(p)$ which is a function of $p \in C^{\prime}$.

Lemma 4.19. (i) The functions $\Psi_{\epsilon}^{*}(p)$ are single valued on $C^{\prime}$, that is, for any cycle $\gamma=\sum_{i=1}^{g}\left(m_{1, i} \mathfrak{a}_{i}+m_{2, i} \mathfrak{b}_{i}\right), m_{1, i}, m_{2, i} \in \mathbb{Z}, \Psi_{\epsilon}^{*}(\gamma p)=\Psi_{\epsilon}^{*}(p)$.

(ii) On $C^{\prime} \backslash\{\infty\} \Psi_{\epsilon}^{*}(p)$ is meromorphic with a simple pole only at $p_{\epsilon}$.

Proof. (i) In a similar way to the proof of Proposition 4.15 (i) the following property can be proved using (4.8) and Proposition 4.12 (i),

$$
\begin{aligned}
& \sigma_{A_{2}^{(g-1)}}^{(g-1)}\left(u(\gamma p)-u\left(p_{\epsilon}\right)\right)=(-1)^{m_{1}^{t} m_{2}+2\left(\kappa^{t} m_{1}-\kappa^{\prime t} m_{2}\right)} \\
& \times \exp \left(2\left(\eta^{(g-1)} m_{1}+\eta^{(g-1)} m_{2}\right)^{t}\left(u(p)-u\left(p_{\epsilon}\right)+\omega^{(g-1)} m_{1}+\omega^{(g-1)} m_{2}\right)\right) \\
& \times \sigma_{A_{2}^{(g-1)}}^{(g-1)}\left(u(p)-u\left(p_{\epsilon}\right)\right)
\end{aligned}
$$

for $\gamma=\sum_{i=1}^{g-1}\left(m_{1, i} \mathfrak{a}_{i}+m_{2, i} \mathfrak{b}_{i}\right)$, where $m_{i}=\left(m_{i, 1}, \ldots, m_{i, g-1}\right)^{t}$. By the definition (4.19) of the differential $\mathrm{d} \widehat{r}_{i}$ the following lemma can be proved. 
Lemma 4.20. 35] The periods of $\mathrm{d} \widehat{r}_{i}$ are given by

$$
\int_{\mathfrak{a}_{j}} \mathrm{~d} \widehat{r}_{i}=\left(\left(2 \eta^{(g-1)}\right)^{t} B^{(g-1)}\right)_{i, j}, \quad \int_{\mathfrak{b}_{j}} \mathrm{~d} \widehat{r}_{i}=\left(\left(2 \eta^{(g-1)}\right)^{t} B^{(g-1)}\right)_{i, j},
$$

Using (4.8), (4.23), Lemma 4.20 we easily have $\Psi_{\epsilon}^{*}(\gamma p)=\Psi_{\epsilon}^{*}(p)$.

By Lemma 4.19 the expansion coefficients of $z^{-(g-2)} \tau_{ \pm}(t) \Psi_{\epsilon}^{*}(t ; z)$ in $t_{1}, t_{2}, \ldots$ is contained in $W_{\epsilon}$ defined by (3.17). By (2.8) the point $U_{\epsilon}$ of UGM corresponding to $\tau_{\epsilon}(t)$ is contained in $W_{\epsilon}$.

Lemma 4.21. If two points $U_{1}, U_{2}$ of UGM satisfy the inclusion relation $U_{1} \subset U_{2}$ then $U_{1}=U_{2}$.

Proof. By $U_{1} \subset U_{2}$ we have

$$
\operatorname{Ker}\left(\left.\pi\right|_{U_{1}}\right) \subset \operatorname{Ker}\left(\left.\pi\right|_{U_{2}}\right), \quad \operatorname{Im}\left(\left.\pi\right|_{U_{1}}\right) \subset \operatorname{Im}\left(\left.\pi\right|_{U_{2}}\right),
$$

and consequently

$$
\begin{aligned}
\operatorname{dim} \operatorname{Ker}\left(\left.\pi\right|_{U_{1}}\right) & \leq \operatorname{dim} \operatorname{Ker}\left(\left.\pi\right|_{U_{2}}\right) \\
\operatorname{dim} \operatorname{Coker}\left(\left.\pi\right|_{U_{1}}\right) & \geq \operatorname{dim} \operatorname{Coker}\left(\left.\pi\right|_{U_{2}}\right) .
\end{aligned}
$$

We show that these inequalities are actually equalities. In fact, if the strict inequality holds in (4.26), for example, then

$$
\operatorname{dim} \operatorname{Ker}\left(\left.\pi\right|_{U_{1}}\right)<\operatorname{dim} \operatorname{Ker}\left(\left.\pi\right|_{U_{2}}\right)=\operatorname{dim} \operatorname{Coker}\left(\left.\pi\right|_{U_{2}}\right) \leq \operatorname{dim} \operatorname{Coker}\left(\left.\pi\right|_{U_{1}}\right)
$$

since $U_{2} \in$ UGM. But it contradicts $U_{1} \in$ UGM.

Now suppose that $U_{1} \neq U_{2}$. Then there exists $v \in U_{2}$ such that $v \notin U_{1}$. If $\pi(v) \neq 0$ then $\operatorname{Im}\left(\left.\pi\right|_{U_{1}}\right) \subsetneq \operatorname{Im}\left(\left.\pi\right|_{U_{2}}\right)$ by (4.25). Therefore $\operatorname{dim} \operatorname{Coker}\left(\left.\pi\right|_{U_{1}}\right)>\operatorname{dim} \operatorname{Coker}\left(\left.\pi\right|_{U_{2}}\right)$ which is impossible as we have just shown. If $\pi(v)=0$, then $\operatorname{Ker}\left(\left.\pi\right|_{U_{1}}\right) \neq \operatorname{Ker}\left(\left.\pi\right|_{U_{2}}\right)$ which is also impossible. Thus $U_{1}=U_{2}$.

By Lemma 4.21 we have $U_{\epsilon}=W_{\epsilon}$. Then there is a constant $c_{\epsilon}$ such that

$$
\tau_{\epsilon}(t)=c_{\epsilon} \tau\left(t ; Y^{\epsilon}\right)
$$

We show that $c_{\epsilon}=1$ by comparing the expansion of tau functions.

By computing the Plücker coordinates of $Y^{\epsilon}$ we easily see that the following expansion holds,

$$
\tau\left(t ; Y^{\epsilon}\right)=s_{\nu^{(g-2)}}(t)+(\text { higher weight terms })
$$

The following proposition is proved in [36].

Proposition 4.22. 36] Suppose that $q \in C \backslash\{\infty\}$. Then

$$
\sigma^{(g-1)}(u+u(q))=\left.c(q) s_{\nu^{(g-2)}}(t)\right|_{t_{2 i-1}=u_{2 i-1}, i \geq 1}+\text { (higher weight terms), }
$$

for some constant $c(q) \neq 0$. 
Notice that $s_{\nu^{(g-2)}}(t)$ depends only on $t_{1}, t_{3}, \ldots, t_{2 g-3}$.

Remark 4.23. In [36] the expansion of the form (4.30) is proved for the sigma function of an arbitrary Riemann surface.

Apply $\partial_{A_{0}^{(g-2)}}$ to (4.30) and set $u=0$. Then By Lemma 4.3 (ii), (iii) we have

$$
\sigma_{A_{0}^{(g-2)}}^{(g-1)}(u(q))=c(q) s_{0}^{(g-2)} .
$$

Therefore

$$
c(q)=s_{0}^{(g-2)} \partial_{A_{0}^{(g-2)}} \sigma^{(g-1)}(u(q)) .
$$

It follows that

$$
c\left(-p_{\epsilon}\right)=C_{\epsilon}^{-1} .
$$

By Proposition 4.22 and (4.31) the expansion of $\tau_{\epsilon}(t)$ takes the form

$$
\tau_{\epsilon}(t)=s_{\nu^{(g-2)}}(t)+\text { (higher weight terms). }
$$

Comparing (4.29) and (4.32) we get $c_{\epsilon}=1$ in (4.28). Thus Theorem 4.18 is proved.

\section{Degeneration of sigma function}

In this section we derive the formula for the degenerate sigma function by substituting the sigma function formula of the tau functions to the relation (3.24).

\subsection{Relation of sigma functions}

Recall that $\tau(t ; \widetilde{X})$ is expressed by the sigma function as in Theorem 4.10 and $\tau\left(t ; \widetilde{X}_{0}\right)$ is a degenerate limit of $\tau(t ; \widetilde{X})$. Since the point of UGM corresponding to $X$ is a gauge transformation of that of the frame $\widetilde{X}_{0}, \tau(t ; X)$ can be expressed by $\tau\left(t ; \widetilde{X}_{0}\right)$. We first derive it.

Expand

$$
\log \left(1-\alpha z^{2}\right)^{-1}=\sum_{j=1}^{\infty}\left(2 \alpha^{j}\right) \frac{z^{2 j}}{2 j}
$$

By (3.14)

$$
\tau(t ; X)=\exp \left(2 \sum_{j=1}^{\infty} \alpha^{j} t_{2 j}\right) \tau\left(t ; \widetilde{X}_{0}\right) .
$$

We denote the limit of $\sigma^{(g)}(u)$ by $\sigma_{g}^{(g) 0}(u)$ etc. by putting the upper index 0 to each quantity. Then, by taking the limit of (4.11) we have

$$
\tau(t ; X)=\exp \left(2 \sum_{j=1}^{\infty} \alpha^{j} t_{2 j}-\sum_{i=1}^{\infty} c_{2 i}^{(g) 0} t_{2 i}+\frac{1}{2} \widehat{q}^{(g) 0}(t)\right) \sigma^{(g) 0}\left(B^{(g) 0} t\right) .
$$

Substitute (4.17) and (5.2) to (3.24). We get 
Theorem 5.1. Let $\sigma^{(g)}(u)$ be the sigma function defined by (4.7) and $\sigma^{(g) 0}(u)$ the limit of $\sigma^{(g)}(u)$ in the limit taking $\alpha_{2 g+1}, \alpha_{2 g}$ to $\alpha$ in the curve $C$ defined by (3.1). Then

$$
\begin{aligned}
& \sigma^{(g) 0}\left(B^{(g) 0} t\right)=\frac{(-1)^{g-1}}{2 y_{0}} \mathrm{~s}_{0}^{(g-2)} \exp \left(-2 \sum_{j=1}^{\infty} \alpha^{j} t_{2 j}+\sum_{i=1}^{\infty} c_{2 i}^{(g) 0} t_{2 i}-\frac{1}{2} \widehat{q}^{(g) 0}(t)+\frac{1}{2} \widehat{q}^{(g-1)}(t)\right) \\
& \times \sum_{\epsilon= \pm} \epsilon \exp \left(\sum_{j=1}^{\infty} d_{j}^{(g-1, \epsilon)} t_{j}\right) \frac{\sigma^{(g-1)}\left(B^{(g-1)} t-u\left(p_{\epsilon}\right)\right)}{\sigma_{A_{0}^{(g-2)}}^{(g-1)}\left(-u\left(p_{\epsilon}\right)\right)},
\end{aligned}
$$

where \pm are identified with $\pm 1, c_{2 i}^{(g)}, B^{(g)}, \widehat{q}^{(g)}(t), d_{j}^{(g, \epsilon)}$ are defined by (4.9), (4.10), (4.16) and $c_{2 i}^{(g) 0}, B^{(g) 0}, \widehat{q}^{(g) 0}(t)$ are the limits of the corresponding quantities.

In this formula the relation between the argument in $\sigma^{(g) 0}$ and that in $\sigma^{(g-1)}$ is not clear. It can be given as follows.

Set

$$
B^{(g) 0} t=\left(u_{1}, u_{3}, \ldots, u_{2 g-1}\right)^{t},
$$

which, in terms of coordinates, is written as

$$
u_{2 i-1}=\sum_{j=1}^{\infty} b_{i, 2 j-1}^{(g) 0} t_{2 j-1} . \quad 1 \leq i \leq g
$$

Then

Lemma 5.2. The following equation holds,

$$
B^{(g-1)} t=\left(u_{1}-\alpha u_{3}, u_{3}-\alpha u_{5}, \ldots, u_{2 g-3}-\alpha u_{2 g-1}\right) .
$$

Proof. The equation (5.5) is equivalent to

$$
\sum_{j=1}^{\infty} b_{i, 2 j-1}^{(g-1)} t_{2 j-1}=u_{2 i-1}-\alpha u_{2 i+1} .
$$

Let us prove it. We have

$$
\begin{aligned}
\mathrm{d} u_{2 i-1}^{(g) 0} & =\frac{z^{2 i-2} \mathrm{~d} z}{\left(1-\alpha z^{2}\right) F_{0}(z)}=\left(\sum_{j=i}^{\infty} b_{i, 2 j-1}^{(g) 0} z^{2 j-2}\right) \mathrm{d} z \\
\mathrm{~d} u_{2 i-1}^{(g-1)} & =\frac{z^{2 i-2} \mathrm{~d} z}{F_{0}(z)}=\left(\sum_{j=i}^{\infty} b_{i, 2 j-1}^{(g-1)} z^{2 j-2}\right) \mathrm{d} z .
\end{aligned}
$$

Therefore, for $1 \leq i \leq g-1$, we have

$$
\mathrm{d} u_{2 i-1}^{(g-1)}=\left(1-\alpha z^{2}\right) \mathrm{d} u_{2 i-1}^{(g) 0},
$$


which is equivalent to

$$
b_{i, 2 j-1}^{(g-1)}=b_{i, 2 j-1}^{(g) 0}-\alpha b_{i, 2 j-3}^{(g) 0},
$$

where we set $b_{i j}^{(g) 0}=0$ if $j \leq 0$. Multiplying $t_{2 j-1}$ and summing up we get

$$
\begin{aligned}
\sum_{j=i}^{\infty} b_{i, 2 j-1}^{(g-1)} t_{2 j-1} & =\sum_{j=i}^{\infty} b_{i, 2 j-1}^{(g) 0} t_{2 j-1}-\alpha \sum_{j=i+1}^{\infty} b_{i, 2 j-3}^{(g) 0} t_{2 j-1} \\
& =u_{2 i-1}-\alpha \sum_{j=i+1}^{\infty} b_{i, 2 j-3}^{(g) 0} t_{2 j-1}
\end{aligned}
$$

Set

$$
\frac{1}{F(z)}=\frac{1}{\left(1-\alpha z^{2}\right) F_{0}(z)}=\sum_{j=0}^{\infty} \beta_{2 j+1} z^{2 j}
$$

By (5.7) we have

$$
b_{i, 2 j-1}^{(g) 0}=\beta_{2 j-2 i+1},
$$

and

$$
u_{2 i-1}=\sum_{j=i}^{\infty} b_{i, 2 j-1}^{(g) 0} t_{2 j-1}=\sum_{j=i}^{\infty} \beta_{2 j-2 i+1} t_{2 j-1} .
$$

Therefore

$$
\sum_{j=i+1}^{\infty} b_{i, 2 j-3}^{(g) 0} t_{2 j-1}=\sum_{j=i+1}^{\infty} \beta_{2 j-2(i+1)+1} t_{2 j-1}=u_{2 i+1} .
$$

Substituting this to (5.10) we get (5.6).

By (5.4) and (5.5) the arguments of $\sigma^{(g) 0}$ and $\sigma^{(g-1)}$ in Theorem 5.1 is directly connected without the variable $t$. Let us study other parts in the formula (5.3).

\subsection{The expression of $t$ by $u$}

In this section we express the variables $t_{i}$ in terms of $\left\{u_{j}\right\}$.

Define

$$
\begin{aligned}
& \Lambda_{n}=\frac{1}{2 n} \sum_{j=1}^{2 g+1} \alpha_{j}^{n}=\frac{1}{2 n}\left(2 \alpha^{2}+\sum_{j=1}^{2 g-1} \alpha_{j}^{n}\right) \\
& \Lambda=\left(\Lambda_{1}, \Lambda_{2}, \Lambda_{3}, \ldots\right)
\end{aligned}
$$

Lemma 5.3. $\beta_{2 j+1}=s_{(j)}(\Lambda), \quad j \geq 0$. 
Proof. We have

$$
\begin{aligned}
\frac{1}{F(z)}=\exp (-\log F(z)) & =\exp \left(-\frac{1}{2} \sum_{j=1}^{2 g+1} \log \left(1-\alpha_{j} z^{2}\right)\right) \\
& =\exp \left(\sum_{n=1}^{\infty}\left(\frac{1}{2 n} \sum_{j=1}^{2 g+1} \alpha_{j}^{n}\right) z^{2 n}\right) \\
& =\exp \left(\sum_{n=1}^{\infty} \Lambda_{n} z^{2 n}\right) \\
& =\sum_{n=0}^{\infty} s_{(n)}(\Lambda) z^{2 n} .
\end{aligned}
$$

Set $t_{j}=0, j \geq 2 g$ and $t_{2 j-1}=0, j \geq g+1$ in (5.4). Then

$$
\left(\begin{array}{c}
u_{1} \\
u_{3} \\
\vdots \\
u_{2 g-1}
\end{array}\right)=\widetilde{M}\left(\begin{array}{c}
t_{1} \\
t_{3} \\
\vdots \\
t_{2 g-1}
\end{array}\right), \quad \widetilde{M}=\left(\begin{array}{ccccc}
1 & \beta_{3} & \beta_{5} & \ldots & \beta_{2 g-1} \\
0 & 1 & \beta_{3} & \ldots & \beta_{2 g-3} \\
0 & \ddots & \ddots & & \vdots \\
\vdots & & \ddots & \ddots & \vdots \\
0 & \ldots & \ldots & 0 & 1
\end{array}\right)
$$

Set

$$
M=\widetilde{M}^{-1} .
$$

The matrix $M$ can be computed as the cofactor matrix.

Lemma 5.4. Equation (5.13) is solved by

$$
\left(\begin{array}{c}
t_{1} \\
t_{3} \\
\vdots \\
t_{2 g-1}
\end{array}\right)=M\left(\begin{array}{c}
u_{1} \\
u_{3} \\
\vdots \\
u_{2 g-1}
\end{array}\right), \quad M=\left(\begin{array}{ccccc}
1 & m_{1,2} & m_{1,3} & \ldots & m_{1, g} \\
0 & 1 & m_{2,3} & \ldots & m_{2, g} \\
0 & \ddots & \ddots & & \vdots \\
\vdots & & \ddots & \ddots & \vdots \\
0 & \ldots & \ldots & 0 & 1
\end{array}\right)
$$

where, for $i<j$,

$$
m_{i j}=(-1)^{i+j} \operatorname{det}\left(\begin{array}{ccccc}
s_{(1)} & s_{(2)} & \ldots & \ldots & s_{(j-i)} \\
1 & s_{(1)} & s_{(2)} & \ldots & s_{(j-i-1)} \\
0 & \ddots & \ddots & & \vdots \\
\vdots & \ddots & \ddots & & \vdots \\
0 & \ldots & \ldots & 1 & s_{(1)}
\end{array}\right), \quad s_{(j)}=s_{(j)}(\Lambda) .
$$




\subsection{Difference of bidifferentials}

In this section we express the coefficients of the quadratic form $-\frac{1}{2} \widehat{q}^{(g) 0}(t)+\frac{1}{2} \widehat{q}^{(g-1)}(t)$ in terms of symmetric functions of $\left\{\alpha_{j}\right\}_{j=1}^{2 g-1}$.

Let

$$
Q_{i, j}=-\widehat{q}_{i, j}^{(g) 0}+\widehat{q}_{i, j}^{(g-1)}, \quad Q=\left(Q_{2 i-1,2 j-1}\right)_{1 \leq i, j \leq g}
$$

and

$$
\begin{aligned}
f_{g}(x) & =(x-\alpha)^{2} \prod_{j=1}^{2 g-1}\left(x-\alpha_{j}\right)=\sum_{i=0}^{2 g+1} \mu_{4 g+2-2 i} x^{i}, \\
f_{g-1}(x) & =\prod_{j=1}^{2 g-1}\left(x-\alpha_{j}\right)=\sum_{i=0}^{2 g-1} \widetilde{\mu}_{4 g-2-2 i} x^{i} .
\end{aligned}
$$

We set $\widetilde{\mu}_{4 g-2-2 i}=0$ if $i<0$ or $i>2 g-1$. The relation

$$
f_{g}(x)=(x-\alpha)^{2} f_{g-1}(x)
$$

implies

$$
\mu_{4 g+2-2 i}=\widetilde{\mu}_{4 g+2-2 i}-2 \alpha \widetilde{\mu}_{4 g-2 i}+\alpha^{2} \widetilde{\mu}_{4 g-2-2 i} .
$$

Define

$$
\begin{aligned}
H_{g}\left(x_{1}, x_{2}\right) & =\sum_{i=0}^{g}\left(x_{1} x_{2}\right)^{i}\left(2 \mu_{4 g+2-4 i}+\mu_{4 g-4 i}\left(x_{1}+x_{2}\right)\right), \\
H_{g-1}\left(x_{1}, x_{2}\right) & =\sum_{i=0}^{g-1}\left(x_{1} x_{2}\right)^{i}\left(2 \widetilde{\mu}_{4 g-2-4 i}+\widetilde{\mu}_{4 g-4-4 i}\left(x_{1}+x_{2}\right)\right) .
\end{aligned}
$$

By computation we have

\section{Lemma 5.5.}

$$
H_{g}\left(x_{1}, x_{2}\right)=\left(x_{1}-\alpha\right)\left(x_{2}-\alpha\right) H_{g-1}\left(x_{1}, x_{2}\right)+\alpha\left(x_{1}-x_{2}\right)^{2} \sum_{i=0}^{g-1} \widetilde{\mu}_{4 g-4-4 i} x_{1}^{i} x_{2}^{i} .
$$

Set $x_{i}=z_{i}^{-2}$ and

$$
y_{i}^{(g-1)}=z_{i}^{-(2 g-1)} F_{0}(z) \quad y_{i}^{(g)}=\left(x_{i}-\alpha\right) y_{i}^{(g-1)}=z_{i}^{-(2 g+1)} F(z) .
$$

Then

$$
\begin{aligned}
\widehat{\Omega}^{(g) 0}\left(x_{1}, x_{2}\right) & =\left(\frac{1}{2\left(x_{1}-x_{2}\right)^{2}}+\frac{H_{g}\left(x_{1}, x_{2}\right)}{4\left(x_{1}-x_{2}\right)^{2} y_{1}^{(g)} y_{2}^{(g)}}\right) \mathrm{d} x_{1} \mathrm{~d} x_{2}, \\
\widehat{\Omega}^{(g-1)}\left(x_{1}, x_{2}\right) & =\left(\frac{1}{2\left(x_{1}-x_{2}\right)^{2}}+\frac{H_{g-1}\left(x_{1}, x_{2}\right)}{4\left(x_{1}-x_{2}\right)^{2} y_{1}^{(g-1)} y_{2}^{(g-1)}}\right) \mathrm{d} x_{1} \mathrm{~d} x_{2} .
\end{aligned}
$$


By Lemma 5.5

$$
\begin{aligned}
-\widehat{\Omega}^{(g) 0}\left(x_{1}, x_{2}\right)+\widehat{\Omega}^{(g-1)}\left(x_{1}, x_{2}\right) & =-\alpha \frac{\sum_{i=0}^{g-1} \widetilde{\mu}_{4 g-4-4 i} x_{1}^{i} x_{2}^{i}}{4 y_{1}^{(g)} y_{2}^{(g)}} \mathrm{d} x_{1} \mathrm{~d} x_{2} \\
& =-\alpha \frac{\sum_{i=0}^{g-1} \widetilde{\mu}_{4 i} z_{1}^{2 i} z_{2}^{2 i}}{F\left(z_{1}\right) F\left(z_{2}\right)} \mathrm{d} z_{1} \mathrm{~d} z_{2} .
\end{aligned}
$$

We have

$$
\frac{1}{F(z)}=\sum_{n=0}^{\infty} s_{(n)}(\Lambda) z^{2 n}
$$

by (5.11) and Lemma 5.3 ,

Compute the expansion of the right hand side of (5.18) using (5.19) and compare the expansion coefficients. Then we get

Proposition 5.6. For $i, j \geq 0$

$$
Q_{2 i+1,2 j+1}=-\alpha \sum_{k=0}^{g-1} \widetilde{\mu}_{4 k} s_{(i-k)} s_{(j-k)},
$$

where $s_{(j)}=s_{(j)}(\Lambda)$.

Example 5.7. The case of $g=2$.

$$
Q_{1,1}=-\alpha, \quad Q_{1,3}=Q_{3,1}=-\alpha s_{(1)}, \quad Q_{3,3}=-\alpha\left(s_{(1)}^{2}+\widetilde{\mu}_{4}\right),
$$

where

$$
s_{(1)}=\alpha-\frac{1}{2} \widetilde{\mu}_{2}
$$

In this case $m_{1,2}=s_{(1)}$ and

$$
t_{1}=u_{1}-s_{(1)} u_{3}, \quad t_{3}=u_{3} .
$$

Then

$$
Q_{1,1} t_{1}^{2}+2 Q_{1,3} t_{1} t_{3}+Q_{3,3} t_{3}^{2}=-\alpha\left(u_{1}^{2}+\widetilde{\mu}_{4} u_{3}^{2}\right) .
$$

\subsection{Final formula for the degenerate sigma function}

Substituting the results in the previous subsections to the formula in Theorem 5.1 we now derive the final formula for the degenerate sigma function.

Set

$$
\begin{aligned}
u & =\left(u_{1}, u_{3}, \ldots, u_{2 g-1}\right)^{t}, \\
\widetilde{u} & =\left(u_{1}-\alpha u_{3}, u_{3}-\alpha u_{5}, \ldots, u_{2 g-3}-\alpha u_{2 g-1}\right)^{t} \\
d^{(g-1, \pm)} & =\left(d_{1}^{(g-1, \pm)}, d_{3}^{(g-1, \pm)}, \ldots, d_{2 g-1}^{(g-1, \pm)}\right)^{t} .
\end{aligned}
$$

Since $\widehat{q}^{(g) 0}(t), \widehat{q}^{(g-1)}(t)$ do not contain $t_{2 j}, j \geq 1$, we set $t_{2 j}=0, j \geq 1$ and $t_{2 j-1}=0$, $j \geq g+1$ in (5.3). Then 
Theorem 5.8. Let $C$ be the hyperelliptic curve $C$ given by (3.1), $\sigma^{(g)}(u)$ the sigma function of $C$ defined by (4.7) and $\sigma^{(g) 0}(u)$ the limit of $\sigma^{(g)}(u)$ when $\alpha_{2 g+1}, \alpha_{2 g} \rightarrow \alpha$. Then $\sigma^{(g) 0}(u)$ is given by the following formula

$$
\begin{aligned}
\sigma^{(g) 0}(u)= & \frac{(-1)^{g-1}}{2 y_{0}} \mathrm{~s}_{0}^{(g-2)} \exp \left(\frac{1}{2} u^{t} M^{t} Q M u\right) \\
& \times \sum_{\epsilon= \pm} \epsilon \exp \left(d^{(g-1, \epsilon) t} M u\right) \frac{\sigma^{(g-1)}\left(\widetilde{u}-u\left(p_{\epsilon}\right)\right)}{\sigma_{A_{0}^{(g-2)}}^{(g-1)}\left(-u\left(p_{\epsilon}\right)\right)}
\end{aligned}
$$

where $s_{0}^{(g-2)}$ is given by (4.6),$M$ is given by (15.15), $Q$ is given by (5.16), (15.20), $d^{(g-1, \epsilon)}$ is given by (5.23), (4.16), $y_{0}, p_{\epsilon}$ are given by (3.16) and $u(p)$ is the Abel-Jacobi map defined by (4.12).

\section{The case of genus two}

In [6] the degeneration of the genus two hyperelliptic sigma function is studied using the linear differential equations satisfied by the sigma function. In this section we derive their formula by our approach.

\subsection{Problem and the strategy}

In [6] some special normalization is imposed on the defining equation of the hyperelliptic curve. By this reason we can not apply the results in this paper directly to the case considered in [6]. Here we first explain what is the problem and how we treat it.

Let $\alpha_{1}, \ldots, \alpha_{5}$ be mutually distinct complex numbers, $a$ a complex number and

$$
\begin{array}{ll}
C_{1}: w^{2}=\prod_{j=1}^{5}\left(v-\alpha_{j}\right)=\sum_{j=0}^{5} \mu_{10-2 j} v^{j}, & \mu_{0}=1, \mu_{2}=0, \\
C_{2}: y^{2}=\prod_{j=1}^{5}\left(x-\widetilde{\alpha}_{j}\right)=\sum_{j=0}^{5} \widetilde{\mu}_{10-2 j} x^{j}, & \widetilde{\alpha}_{j}=\alpha_{j}+\frac{2}{3} \alpha,
\end{array}
$$

genus two hyperelliptic curves. Two curves $C_{1}$ and $C_{2}$ are isomorphic by

$$
(x, y) \longrightarrow(v, w)=\left(x-\frac{2}{3} \alpha, y\right) .
$$

Assume that $\alpha \neq \alpha_{j}, j=1,2,3$ and consider the degeneration $\alpha_{4}, \alpha_{5} \rightarrow \alpha$ of the curve $C_{1}$ to

$$
w^{2}=(v-\alpha)^{2} \prod_{j=1}^{3}\left(v-\alpha_{j}\right)=\sum_{j=0}^{5} \mu_{10-2 j}^{0} v^{j} .
$$

Correspondingly the equation (6.2) degenerates to

$$
y^{2}=\left(x-\frac{5}{3} \alpha\right)^{2}\left(x^{3}-\frac{g_{2}}{4} x-\frac{g_{3}}{4}\right)
$$


where we set

$$
g_{2}=-4\left(\widetilde{\alpha}_{1} \widetilde{\alpha}_{2}+\widetilde{\alpha}_{1} \widetilde{\alpha}_{3}+\widetilde{\alpha}_{2} \widetilde{\alpha}_{3}\right), \quad g_{3}=4 \widetilde{\alpha}_{1} \widetilde{\alpha}_{2} \widetilde{\alpha}_{3} .
$$

Notice that the coefficient of $x^{2}$ becomes zero in the second factor of the right hand side of (6.5). This is the reason why we consider the shift by $(2 / 3) \alpha$ in (6.2). Notice that, due to $\mu_{2}=0$, the coefficients of $\prod_{j=1}^{3}\left(v-\alpha_{j}\right)$ in (6.4) becomes $2 \alpha$ which is not zero if $\alpha \neq 0$.

In [6] the limit of the sigma function of $C_{1}$ is expressed by the sigma function of the elliptic curve

$$
C_{3}: y^{2}=x^{3}-\frac{g_{2}}{4} x-\frac{g_{3}}{4} .
$$

Although $C_{1}$ and $C_{2}$ are isomorphic, their sigma functions are not the same (see Remark 6.2). So we can not apply our result directly to the case studied in [6]. However the idea to use tau function can be applied. The strategy is as follows. We first show that the tau functions of $C_{1}$ and $C_{2}$ are the same. By Theorem 3.2 the limit of the tau function of $C_{2}$ is expressed by the tau functions of $C_{3}$. We can not apply Theorem 4.10 to the tau functions of $C_{1}$, since the sigma function formula of the tau function depends on the choice of a local coordinate. As we shall see, for $C_{1}$, we need to use the local coordinate $z$ at $\infty$ given by

$$
v=\frac{1}{z^{2}}-\frac{2}{3} \alpha
$$

While Theorem 4.10 is derived using the local coordinate $z$ such that

$$
v=\frac{1}{z^{2}} \text {. }
$$

However it is possible to make the sigma function formula of the tau function using the local coordinate satisfying $(6.8)$ in a completely similar way to the case of (6.9) .

\subsection{Tau function of $C_{1}$ and $C_{2}$}

In this section we show that the tau function of $C_{1}$ and that of $C_{2}$ coincide.

Take the local coordinate $z$ at $\infty$ of $C_{2}$ by

$$
x=\frac{1}{z^{2}}, \quad y=\frac{1}{z^{5}}(1+O(z)) .
$$

By (6.3) $z$ becomes a local coordinate at $\infty$ of $C_{1}$ such that

$$
v=\frac{1}{z^{2}}-\frac{2}{3} \alpha, \quad w=\frac{1}{z^{5}}(1+O(z)) .
$$

By (3.3), (3.4) a basis of $H^{0}\left(C_{2}, \mathcal{O}(* \infty)\right)$ is given by

$$
z^{-2 i}, \quad z^{-5-2 i} F(z), \quad i \geq 0,
$$

where

$$
F(z)=\left(\prod_{j=1}^{5}\left(1-\widetilde{\alpha}_{j} z^{2}\right)\right)^{\frac{1}{2}}
$$


A basis of $H^{0}\left(C_{1}, \mathcal{O}(* \infty)\right)$ is given by

$$
v^{i}, \quad v^{i} w, \quad, i \geq 0
$$

which, in terms of $z$, is written as

$$
\left(z^{-2}-\frac{2}{3} \alpha\right)^{i}, \quad z^{-5}\left(z^{-2}-\frac{2}{3} \alpha\right)^{i} F(z), \quad i \geq 0 .
$$

We easily have

Lemma 6.1. The vector space generated by (6.11) and that by (6.12) coincide.

By Lemma 6.1 $C_{1}$ and $C_{2}$ with the local coordinate $z$ determine the same frame $\tilde{X}$ given by (3.7). Therefore their tau functions are the same. Consequently Theorem 3.2 holds for tau functions of $C_{1}$ and $C_{3}$.

\section{3 $\tau(t ; X)$ by sigma function of $C_{1}$}

In this section we express $\tau(t ; X)$, which is a gauge transformation of the degenerate limit of $\tau(t ; \widetilde{X})$, in terms of the degenerate limit of the sigma function of $C_{1}$.

Redefine $b_{i, j}^{(g)}, \widehat{q}_{i, j}^{(g)}, c_{i}^{(g)}$ by the expansion coefficients in terms of the local coordinate $z$ of (6.10). Then it can be proved that Theorem 4.10 holds without any change, where the sigma function in the right hand side is that defined from $C_{1}$. In order to compare the formula in [6] we express the limits of $b_{i, j}^{(g)}, \widehat{q}_{i, j}^{(g)}$ in terms of the quantities associated with the curve $C_{3}$.

Let $\wp(u)$ be the Weierstrass elliptic function. The points of $C_{3}$ are parametrized as

$$
(x, y)=\left(\wp(u), \frac{1}{2} \wp^{\prime}(u)\right), \quad u \in \mathbb{C} .
$$

By definition

$$
p_{ \pm}=\left(\frac{5}{3} \alpha, \pm y_{0}\right) \in C_{3}
$$

We choose $a \in \mathbb{C}$ such that

$$
p_{ \pm}=\left(\wp(a), \frac{1}{2} \wp^{\prime}( \pm a)\right)
$$

which means

$$
\alpha=\frac{3}{5} \wp(a), \quad y_{0}=\frac{1}{2} \wp^{\prime}(a)
$$

For the curve $C_{3}$

$$
\mathrm{d} u_{1}^{(1)}=-\frac{\mathrm{d} x}{2 y} .
$$


Then

$$
u\left(p_{ \pm}\right)=\int_{\infty}^{p_{ \pm}} \mathrm{d} u_{1}^{(1)}=-\int_{\infty}^{p_{ \pm}} \frac{\mathrm{d} x}{2 y}=-( \pm a)=\mp a .
$$

In the following everything is computed in the limit $\alpha_{4}, \alpha_{5} \rightarrow \alpha$. We have

$$
\begin{aligned}
\mathrm{d} u_{1}^{(2) 0} & =-\frac{v \mathrm{~d} v}{2 w}=\left(1+\alpha z+O\left(z^{4}\right)\right) d z, \\
\mathrm{~d} u_{3}^{(2) 0} & =-\frac{\mathrm{d} v}{2 w}=\left(z^{2}+O\left(z^{4}\right)\right) d z
\end{aligned}
$$

Thus

$$
B^{(2) 0}=\left[\begin{array}{cccccc}
1 & 0 & \alpha & 0 & * & \ldots \\
0 & 0 & 1 & 0 & * & \ldots
\end{array}\right] .
$$

By definition

$$
\begin{aligned}
\widehat{\Omega}^{(2) 0}\left(p_{1}, p_{2}\right) & =\frac{2 w_{1} w_{2}+\sum_{i=0}^{2} v_{1}^{i} v_{2}^{i}\left(2 \mu_{10-4 i}^{(2) 0}+\mu_{8-4 i}^{(2) 0}\left(v_{1}+v_{2}\right)\right)}{4\left(v_{1}-v_{2}\right)^{2} w_{1} w_{2}} \mathrm{~d} v_{1} \mathrm{~d} v_{2} \\
& =\left(\frac{1}{\left(z_{1}-z_{2}\right)^{2}}+\sum_{i, j \geq 1} \widehat{q}_{2 i-1,2 j-1}^{(2) 0} z_{1}^{2(i-1)} z_{2}^{2(i-1)}\right) \mathrm{d} z_{1} \mathrm{~d} z_{2} .
\end{aligned}
$$

Then

$$
\begin{aligned}
& \widehat{q}_{1,1}^{(2) 0}=\frac{1}{5} \wp(a), \quad \widehat{q}_{1,3}^{(2) 0}=\widehat{q}_{3,1}^{(2) 0}=\frac{9}{25} \wp(a)^{2}+\frac{g_{3}}{8}, \\
& \widehat{q}_{3,3}^{(2) 0}=-\frac{3 g_{2}}{20} \wp(a)+\frac{3 g_{3}}{8}+\frac{63}{125} \wp(a)^{3} .
\end{aligned}
$$

Similarly to (4.9) $c_{2 i-1}^{(2) 0}=0, i \geq 1$. If

$$
t_{2 j}=t_{2 j+3}=0, \quad j \geq 1,
$$

we have

$$
\begin{aligned}
B^{(2) 0} t & =\left[\begin{array}{c}
t_{1}+\alpha t_{3} \\
t_{3}
\end{array}\right] \\
\widehat{q}^{(2) 0}(t) & =\sum_{i, j=1}^{2} \widehat{q}_{2 i-1,2 j-1}^{(2) 0} t_{2 i-1} t_{2 j-1} .
\end{aligned}
$$

Therefore, taking (5.1) into account, we have

$$
\tau\left(\left(t_{1}, 0, t_{3}, 0, \ldots\right)^{t} ; X\right)=\exp \left(\frac{1}{2} \widehat{q}^{(2) 0}(t)\right) \sigma^{(2) 0}\left(t_{1}+\alpha t_{3}, t_{3}\right) .
$$




\subsection{Tau function $\tau\left(t ; Y^{ \pm}\right)$by sigma function of $C_{3}$}

In this section we derive the expression of $\tau\left(t ; Y^{ \pm}\right)$in terms of the sigma function of $C_{3}$.

Put $g=2$ in Theorem 4.18 and use $s_{0}^{(0)}=1, A_{0}^{(0)}=\emptyset$. Then

$$
\begin{aligned}
\tau\left(\left(t_{1}, 0, t_{3}, 0, \ldots\right)^{t} ; Y^{ \pm}\right) \\
\quad=\exp \left(\sum_{j=1,3} d_{j}^{(1, \pm)} t_{j}+\frac{1}{2} \widehat{q}^{(1)}(t)\right) \frac{\sigma\left(B^{(1)} t-u\left(p_{ \pm}\right)\right)}{\sigma\left(-u\left(p_{ \pm}\right)\right)}
\end{aligned}
$$

where $\sigma(u)=\sigma^{(1)}(u)$ is the Weierstrass sigma function.

We have

$$
\mathrm{d} u_{1}^{(1)}=-\frac{\mathrm{d} x}{2 y}=\left(1+O\left(z^{4}\right)\right) \mathrm{d} z
$$

and

$$
\begin{aligned}
& B^{(1)}=[1,0,0,0, *, \ldots] . \\
& B^{(1)} t=t_{1} .
\end{aligned}
$$

For the bidifferential of $C_{3}$ we have

$$
\begin{aligned}
& \widehat{\Omega}^{(1)}\left(p_{1}, p_{2}\right) \\
= & \frac{2 y_{1} y_{2}+x_{1} x_{2}\left(x_{1}+x_{2}\right)-\frac{1}{4} g_{2}\left(x_{1}+x_{2}\right)-\frac{1}{2} g_{3}}{4\left(x_{1}-x_{2}\right)^{2} y_{1} y_{2}} \mathrm{~d} x_{1} \mathrm{~d} x_{2} \\
= & \left(\frac{1}{\left(z_{1}-z_{2}\right)^{2}}+\frac{g_{2}}{8}\left(z_{1}^{2}+z_{2}^{2}\right)+\frac{g_{3}}{8}\left(z_{1}^{4}+z_{2}^{4}+3 z_{1}^{2} z_{2}^{2}\right)+\cdots\right) \mathrm{d} z_{1} \mathrm{~d} z_{2} .
\end{aligned}
$$

Therefore

$$
\widehat{q}_{1,1}^{(1)}=0, \quad \widehat{q}_{1,3}^{(1)}=\widehat{q}_{3,1}^{(1)}=\frac{g_{2}}{8}, \quad \widehat{q}_{3,3}^{(1)}=\frac{3 g_{3}}{8} .
$$

By (4.16) and (6.15)

$$
\log \left(\frac{\sigma( \pm a)}{\sigma(p \pm a)}\right)-\frac{1}{2} \widehat{q}^{(1)}([z] \mid[z])=\sum_{j=1}^{\infty} d_{j}^{(1, \pm)} \frac{z^{j}}{j} .
$$

Since $\widehat{q}^{(1)}([z] \mid[z])$ contains only even powers of $z, d_{j}^{(1, \pm)}, j=1,3$ are the expansion coefficients of the first term of the left hand side of (6.25). By computation we have

$$
d_{1}^{(1, \pm)}=\mp \zeta(a), \quad d_{3}^{(1, \pm)}= \pm \frac{1}{2} \wp^{\prime}(a),
$$

where $\zeta(u)$ is the Weierstrass zeta function. Substituting (6.23), (6.26) and (6.15) into (6.22) we have

$$
\begin{aligned}
\tau\left(\left(t_{1}, 0, t_{3}, 0, \ldots\right)^{t} ; Y^{ \pm}\right) \\
\quad=\exp \left(\mp \zeta(a) t_{1} \pm \frac{1}{2} \wp^{\prime}(a) t_{3}+\frac{1}{2} \widehat{q}^{(1)}(t)\right) \frac{\sigma\left(t_{1} \pm a\right)}{\sigma( \pm a)} .
\end{aligned}
$$




\subsection{Genus two degenerate formula}

In this section we derive the formula of [6] based on the results of previous subsections.

Substitute (6.21) and (6.27) into (3.24) in Theorem 3.2, use (6.14) and solve in $\sigma^{(2) 0}$. Then we have

$$
\begin{aligned}
& \sigma^{(2) 0}\left(t_{1}+\alpha t_{3}, t_{3}\right) \\
= & \frac{-1}{\wp^{\prime}(a)} \exp \left(\frac{1}{2}\left(-q^{(2) 0}(t)+q^{(1)}(t)\right)\right) \\
& \times \sum_{\epsilon= \pm 1} \epsilon \exp \left(-\epsilon \zeta(a) t_{1}+\epsilon \frac{1}{2} \wp^{\prime}(a) t_{3}\right) \frac{\sigma\left(t_{1}+\epsilon a\right)}{\sigma(\epsilon a)} .
\end{aligned}
$$

Set

$$
u_{1}=t_{1}+\alpha t_{3} . \quad u_{3}=t_{3} .
$$

Solving in $x$ we have

$$
t_{1}=u_{1}-\alpha u_{3}, \quad t_{3}=u_{3} .
$$

Then

$$
\begin{aligned}
\frac{1}{2}\left(-q^{(2) 0}(t)+q^{(1)}(t)\right)= & -\frac{1}{10} \wp(a) u_{1}^{2}-\frac{6}{25} \wp(a)^{2} u_{1} u_{3} \\
& +\left(-\frac{9}{125} \wp(a)^{3}+\frac{3}{40} g_{2} \wp(a)\right) u_{3}^{2}, \\
-\zeta(a) t_{1}+\frac{1}{2} \wp(a) t_{3}= & -\zeta(a) u_{1}+\left(\frac{3}{5} \zeta(a) \wp(a)+\frac{1}{2} \wp^{\prime}(a)\right) u_{3} .
\end{aligned}
$$

Substitute (6.30), (6.31), (6.32) into (6.28) we get, with $u=\left(u_{1}, u_{3}\right)^{t}$,

$$
\begin{aligned}
\sigma^{(2) 0}(u)= & \frac{-1}{\sigma(a) \wp^{\prime}(a)} \exp \left(-\frac{3}{5} \wp(a)\left(\frac{1}{6} u_{1}^{2}+\frac{2}{5} \wp(a) u_{1} u_{3}+\left(\frac{3}{25} \wp(a)^{2}-\frac{g_{2}}{8}\right) u_{3}^{2}\right)\right) \\
& \times \sum_{\epsilon= \pm 1} \exp \left(\epsilon\left(-\zeta(a) u_{1}+\left(\frac{3}{5} \zeta(a) \wp(a)+\frac{1}{2} \wp^{\prime}(a)\right) u_{3}\right)\right) \\
& \times \sigma\left(u_{1}-\frac{3}{5} \wp(a) u_{3}+\epsilon a\right),
\end{aligned}
$$

which coincides with the formula in Theorem 1 of [6] up to sign. The discrepancy of the sign is due to the different normalization of the sigma function.

Remark 6.2. Recall that the left hand side of (6.33) is the degenerate sigma function of the curve $C_{1}$. The formula of Theorem 5.8 gives a formula for the degenerate sigma function of the curve $C_{2}$. By Example $5.7 \mathrm{it}$ is obtained in (6.33) by replacing the exponent of the exponential in front of the sum symbol by

$$
-\frac{5}{6} \alpha\left(u_{1}^{2}+\widetilde{\mu}_{4} u_{3}^{2}\right)
$$

It shows that the sigma functions of $C_{1}$ and $C_{2}$ do not coincide. 


\section{Acknowledegements}

We thank the anonymous referees for careful reading the manuscript and for their comments that helped us to significantly improve the manuscript. This work was supported by JSPS KAKENHI Grant Number JP15K04907.

\section{References}

[1] S. Abenda and P. Grinevich, Rational degenerations of M-curves, totally positive Grassmannians and KP-solitons, Comm. Math. Phys. 361 (2018) 1029-1081, https://doi:10.1007/s00220-018-3123-y.

[2] T. Ayano, Sigma functions for telescopic curves, Osaka J. Math. 51-2 (2014), 459481.

[3] T. Ayano and A. Nakayashiki, On Addition Formulae for Sigma Functions of Telescopic Curves, Symmetry, Integrability and Geometry: Methods and Applications (SIGMA) 9 (2013), 046, 14 pages.

[4] H. F. Baker, Abelian functions. Abel's theorem and the allied theory of theta functions, 1898 Cambridge Mathematical Library, Cambridge University Press, Cambridge, 1995.

[5] E. Belokolos, A. Bobenko, V. Enolski, A. Its and V. Matveev, Algebro-Geometric approach to nonlinear integrable equations, Springer, 1994.

[6] J. Bernatska and D. Leykin, On degenerate sigma-functions in genus two, Glasgow Math. J. 61-1 (2019), 169-193.

[7] V. M. Buchstaber, V. Z. Enolski and D. V. Leykin, Kleinian functions, hyperelliptic Jacobians and applications, in Reviews in Math. and Math. Phys. 10, No.2, Gordon and Breach, London, 1997, 1-125.

[8] V.M. Buchstaber, V.Z. Enolski and D.V. Leykin, Rational analogue of Abelian functions, Funct. Annal. Appl. 33-2 (1999), 83-94.

[9] V. M. Buchstaber, V. Z. Enolski and D. V. Leykin, Multi-Dimensional SigmaFunctions, arXiv:1208.0990.

[10] V. M. Buchstaber, V. Z. Enolski and D. V. Leykin, Multi-variable sigma-functions: old and new results, in Integrable Systems and Algebraic Geometry, R. Donagi, T. Shaska (eds.) LMS Lecture Notes Series. Volume 2, Cambridge University Press, in press, arXiv: 1810.11079 .

[11] V. M. Buchstaber and D. V. Leykin, Addition Laws on Jacobian Varieties of Plane Algebraic Curves. Proceedings of the Steklov Institute of Mathematics, 251 (2005), pp. 172. Translated from Trudy Matematicheskogo Instituta imeni V.A. Steklova, 251, (2005), pp. 54-126. 
[12] V. M. Buchstaber, D. V. Leykin and V. Z. Enolski, Uniformization of Jacobi Varieties of Trigonal Curves and Nonlinear Differential Equations. Funct.Anal.Appl. (2000) 34, Issue $3,159-171$.

[13] E. Date, M. Kashiwara, M. Jimbo, and T. Miwa, Transformation groups for soliton equations, in "Nonlinear Integrable Systems - Classical Theory and Quantum Theory", M. Jimbo and T. Miwa (eds.), World Sci., Singapore, 1983, pp.39-119.

[14] J. C. Eilbeck, V. Z. Enolski and J. Gibbons, Sigma, tau and Abelian functions of algebraic curves, J. Phys. A: Math. Theor. 43 (2010), 455216.

[15] J. C. Eilbeck, V. Z. Enolski, S. Matsutani, Y. Ônishi and E. Previato, Abelian functions for trigonal curves of genus three, Int. Math. Res. Not. 2007, (2007): rnm 140-68.

[16] V. Enolski and J. Harnad, Schur function expansions of KP tau functions associated with algebraic curves. (Russian) Uspekhi Mat. Nauk 66 (2011), no. 4(400), 137-178; translation in Russian Math. Surveys 66 (2011), no. 4, 767-807.

[17] B. Eynard, Notes about a combinatorial expression of the fundamental second kind differential on an algebraic curve, arXiv:1805.07247.

[18] J. Fay, Theta-Functions on Riemann Surfaces, Springer Lecture Notes in Mathematics 352, 1973.

[19] H.M. Farkas and I. Kra, Riemann Surfaces, Second Edition, Springer-Verlag, 1992.

[20] B-F, Feng, L. Ling and D.A. Takahashi, Multi-breathers and high order rogue waves for the nonlinear Schrödinger equation on the elliptic function background, arXiv:1812.04212.

[21] P.G. Grinevich and P.M. Santini, The finite gap method and the periodic NLS Cauchy problem for the anomalous waves, for a finite number of unstable modes, arXiv:1810.09247.

[22] D.J. Kedziora, A. Ankiewicz and N. Akhmediev, Rogue waves and solitons on a cnoidal background, European Phys. J. 223 (2014), 43-62.

[23] N. Kawamoto, Y. Namikawa, A. Tsuchiya and Y. Yamada, Geometric realization of conformal field theory on Riemann surafces, Comm. Math. Phys. 116 (1988) 247-308.

[24] F. Klein, Ueber hyperelliptische Sigmafunctionen, Math. Ann. 27 (1886),341-464.

[25] F. Klein, Ueber hyperelliptische Sigmafunctionen (Zweiter Aufsatz), Math. Ann. 32 (1888), 351-380.

[26] D. Korotkin and V. Shramchenko, On higher genus Weierstrass sigma-function, Physica D: Nonlinear Phenomena, Vol. 241, 23-24 (2012), 2086-2094.

[27] I. G. Macdonald, Symmetric Functions and Hall Polynomials, second edition, Oxford University Press, 1995. 
[28] M. Mulase, Algebraic theory of the KP equations, in Perspectives in Math. Phys., R.Penner and S.T.Yau (eds.), International Press Company, (1994), 157-223.

[29] Y. Kodama, KP solitons and the Grassmannians, Springer, 2017.

[30] I.M. Krichever, Methods of algebraic geometry in the theory of nonlinear equations, Russ. Math. Surv., Vol. 32 (1977), 185-213.

[31] S. Matsutani and E. Previato, Jacobi inversion on strata of the Jacobian of the $C_{r s}$ curve $y^{r}=f(x)$, I,II J. Math. Soc. Japan, 60, No. 4 (2008), 1009-1044. 66, No. 2 (2014), 647-692.

[32] Mumford, D., Tata lectures on theta I, Birkhauser, 1983.

[33] D. Mumford, Tata lectures on theta II, Birkhauser, 1983.

[34] A. Nakayashiki, On algebraic expressions of sigma functions for $(n, s)$ curves, Asian J. Math. 14 (2010), 175-211.

[35] A. Nakayashiki, Sigma function as a tau function, Int. Math. Res. Not. IMRN 2010-3 (2010), 373-394.

[36] A. Nakayashiki, Tau function approach to theta functions, Int. Math. Res. Not. IMRN 2016-17 (2016), 5202-5248.

[37] A. Nakayashiki, Degeneration of trigonal curves and solutions of the KP-hierarchy, Nonlinearity 31 (2018), 3567-3590.

[38] A. Nakayashiki, On reducible degeneration of hyperelliptic curves and soliton solutions, SIGMA 15 (2019), 009, 18 pages.

[39] A. Nakayashiki and K. Yori, Derivatives of Schur, tau and sigma functions, on AbelJacobi images, in Symmetries, Integrable Systems and Representations, K.Iohara, S. Morier-Genoud, B. Remy (eds.), Springer, 2012, 429-462.

[40] Y. Ônishi, Determinant expressions for hyperelliptic functions, with an Appendix by Shigeki Matsutani: Connection of the formula of Cantor and Brioschi-Kiepert type, Proc. Edinburgh Math. Soc. 48 (2005), 705-742.

[41] M. Sato and Y. Sato, Soliton equations as dynamical systems on infinite dimensional Grassmann manifold, in "Nolinear Partial Differential Equations in Applied Sciences", P. D. Lax, H. Fujita and G. Strang (eds.), North-Holland, Amsterdam, and Kinokuniya, Tokyo, 1982, pp.259-271.

[42] J. Suzuki, Klein's Fundamental 2-Form of Second Kind for the $C_{a b}$ Curves Symmetry, Integrability and Geometry: Methods and Applications (SIGMA) 13 (2017), 017, 13 pages

[43] G. Segal and G. Wilson, Loop groups and equations of KdV type, Publ. Math. IHES $61(1985) 5-65$. 